\title{
The Earwig Collection (Dermaptera) of the Manchester Museum, UK, with a complete type catalogue
}

\author{
Claire MILES \\ Department of Entomology, The Manchester Museum, University of Manchester, Oxford Road, \\ Manchester, M13 9PL, UK. Email: claire.miles@manchester.ac.uk \\ urn:1sid:zoobank.org:author:527E8319-57C4-4C8D-8896-46FA09795B2E
}

\section{Table of Contents}

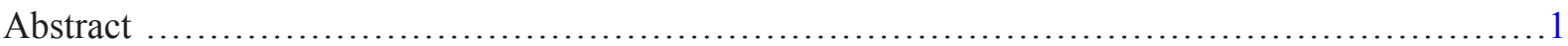

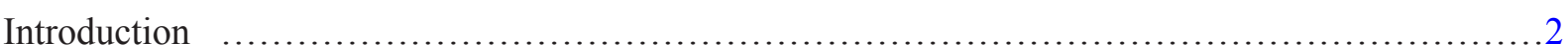

Description of the Dermaptera collection ....................................................

Development of the Dermaptera collection .............................................. 3

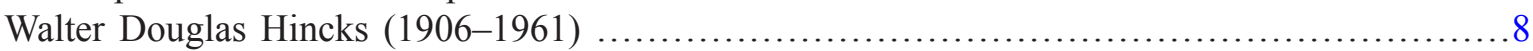

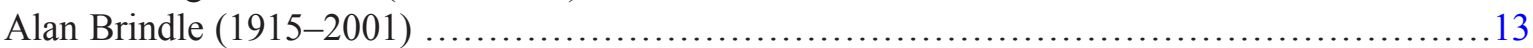

The Entomological Archive ............................................................... 15

Notable earwig specimens in the MMUE collection ....................................... 16

Giant Earwig, Labidura herculeana (Fabricius, 1798) ......................................

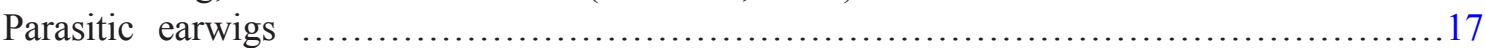

Type catalogue of the Dermaptera collection at MMUE ....................................... 20

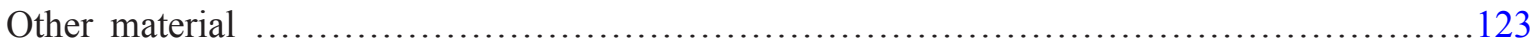

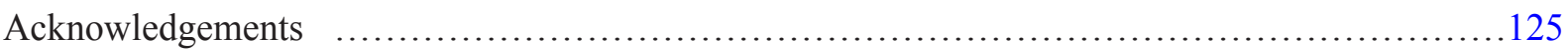

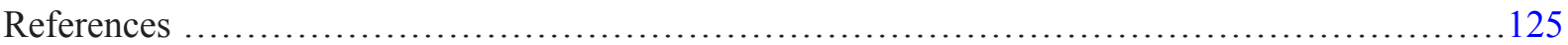

Appendix I: Correspondence in the Hincks archive relating to Dermaptera ...................134

Appendix II: Correspondence in the Brindle archive relating to Dermaptera ...................137

Abstract. This paper presents a description of the worldwide Dermaptera collection of the Manchester Museum, UK. In total, the collection contains over 16,700 specimens and represents 835 species and subspecies (approximately 44\% of the Dermaptera world fauna). The composition of the collection is described and a brief account is given of its history and those instrumental in its development, notably W.D. Hincks (1906-1961) and A. Brindle (1915-2001). Correspondence held at the museum relating to their work on Dermaptera is listed and examples relating to Labidura herculeana (Fabricius, 1798) and some of the parasitic earwigs in the collection are explored. Finally, a complete type catalogue of the Manchester Museum's Dermaptera is presented; it is arranged alphabetically by species name as originally described, with photographs of all holotypes and their corresponding data labels. The collection contains 40 holotypes and 694 secondary types (allotypes, syntypes or paratypes), representing 283 species and subspecies, an estimated $15 \%$ of the Dermaptera world fauna.

Key words. Dermaptera, type catalogue, Manchester Museum, W.D. Hincks, A. Brindle.

Miles C. 2015. The Earwig Collection (Dermaptera) of the Manchester Museum, UK, with a complete type catalogue. European Journal of Taxonomy 141: 1-138. http://dx.doi.org/10.5852/ejt.2015.141 


\section{Introduction}

The collections of arthropods at the Manchester Museum (MMUE) are among the top three in the UK, containing more than three million specimens, including about two and a half million insects (Logunov \& Merriman 2012; Logunov 2010a). The worldwide Dermaptera collection is one of the particular strengths of the Entomology Department and it is probably among the most comprehensive in the world (Logunov 2010b), containing almost a half of the species described worldwide (see Table 1).

During the review of the collection, an updated count was made of all its constituent parts (Table 1). The total of over 16,700 specimens is approximately 5,000 more than previously thought (Logunov \& Merriman 2012). In the process, accession numbers have been added to all individual dried, mounted primary and secondary type specimens and to all vials of spirit specimens.

The aims of this paper are (1) to briefly describe the composition of the Museum's Dermaptera collection and its history and (2) to provide a complete catalogue of its type material. The Manchester collections are searchable online at http://www.museum.manchester.ac.uk; however, the Dermaptera collection is not yet completely databased on the Museum's collection management system to species level and this significant resource is currently very little used. It is hoped that the publication of this paper with a complete type catalogue will encourage and facilitate the use of this important, but underpublicized and underused collection.

\section{Description of the Dermaptera collection}

The collection comprises over 16,700 specimens organised in five divisions:

1. Approximately 10,800 worldwide specimens (accession no. F3233) are dry mounted, housed in three Watkins and Doncaster wooden cabinets, each with 20 glass-lidded drawers (Fig. 1). The specimens are pinned or mounted on card and arranged taxonomically. Table 1 gives the numerical composition of the collection and Fig. 2 gives the percentage of World Dermaptera represented at the Manchester Museum, by family. Three drawers (approx. 1300 specimens) contain undetermined material, partially sorted according to the zoogeographical regions. Table 2 shows the geographical distribution of the undetermined material.

2. The British earwig collection is kept separate from the foreign insects and is housed in one drawer of a Hill unit (Fig. 3). The four native species are represented, as well as Euborellia annulipes (Lucas, 1847) and Marava arachidis (Yersin, 1860), with 250 specimens in total (Table 3). In addition, there is one specimen of Labidura riparia (Pallas, 1773) from France in this collection.

3. There are around 2000 undetermined specimens of papered material. 1600 are Indian Dermaptera (accession no. F3254) collected between 1953 and 1972 (Fig. 4). They were supplied by the wellknown Indian entomologist and natural history dealer P. Susai Nathan (1891-1976) of Kurumbagaram (the 'P.' is the initial of his father's name, Prabala Nathan; M.A.N. Jayapaul pers. comm., 2015). Following his retirement in 1969, his daughter-in-law Theresa Rajabi Susai Nathan took over the dealership (see comments in the text under 'Alan Brindle'). 185 specimens (accession no. F3241) are from South America, collected by Czeslaw M. Biezanko (1895-1986), a Polish entomologist and recognized authority on South American butterflies. A further 200 specimens are from northern India collected between 1953 and 1956. The collector is identified only as 'F.S'.

4. The spirit collection contains approximately 3500 Dermaptera specimens stored in $70 \%$ industrial methylated spirit in glass tubes with polythene caps. The tubes are stored upright in plastic boxes lined with foam plastic. While completing this project, the opportunity was taken to re-curate the spirit collection by topping up where necessary, restoring specimens which had completely dried 
MILES C., Dermaptera of the Manchester Museum

Table 1. Composition of the Manchester Museum's Dermaptera collection.

\begin{tabular}{|c|c|c|c|c|c|c|c|c|}
\hline \multirow[b]{3}{*}{ Family } & \multicolumn{4}{|c|}{ No. of species and subspecies } & \multirow{3}{*}{$\begin{array}{c}\text { MMUE's } \\
\text { representation } \\
\text { of World } \\
\text { Fauna (\%) }\end{array}$} & \multirow{2}{*}{\multicolumn{3}{|c|}{ No. of specimens }} \\
\hline & \multirow[b]{2}{*}{$\begin{array}{l}\text { World } \\
\text { Fauna }^{1}\end{array}$} & \multicolumn{3}{|c|}{ MMUE collection } & & & & \\
\hline & & Dry & Spirit & $\begin{array}{l}\text { Combined } \\
\text { Dry/Spirit }^{2}\end{array}$ & & Dry & Spirit & Total \\
\hline Anisolabididae & 393 & 170 & 16 & 170 & 43.3 & 1426 & 532 & 1958 \\
\hline Apachyidae & 15 & 8 & 0 & 8 & 53.3 & 72 & 0 & 72 \\
\hline Arixeniidae & 5 & 2 & 2 & 2 & 40.0 & 9 & 20 & 29 \\
\hline Chelisochidae & 95 & 32 & 1 & 32 & 33.7 & 463 & 1 & 464 \\
\hline Diplatyidae & 144 & 43 & 0 & 43 & 29.9 & 136 & 0 & 136 \\
\hline Forficulidae & 470 & 185 & 21 & 185 & 39.4 & 3077 & 820 & 3897 \\
\hline Hemimeridae & 11 & 5 & 3 & 6 & 54.5 & 21 & 15 & 36 \\
\hline Karschiellidae & 12 & 11 & 0 & 11 & 91.7 & 88 & 0 & 88 \\
\hline Labiduridae & 76 & 36 & 4 & 36 & 47.7 & 1044 & 209 & 1253 \\
\hline Pygidicranidae & 185 & 92 & 1 & 92 & 49.7 & 734 & 1 & 735 \\
\hline Spongiphoridae & 510 & 250 & 17 & 250 & 49.0 & 2825 & 1386 & 4211 \\
\hline Undetermined & - & - & - & - & - & $3326^{3}$ & 544 & 3870 \\
\hline Total & 1916 & 834 & 65 & 835 & 43.6 & 13,221 & 3528 & 16,749 \\
\hline
\end{tabular}

${ }^{1}$ Hopkins et al. (2014) (Extant species).

${ }^{2}$ Species present in both dry and spirit collections counted only once.

${ }^{3}$ Includes both mounted and papered material.

out, and adding accession numbers to all vials. There are two species in the spirit collection not represented in the dry collection. One is Hemimerus hanseni Sharp, 1895. The other is a species for which the author was unable to find reference to a published description, Pericomus nigra, identified by Brindle in 1980. The specimens of this species were part of around 5000 collected by Dr. Henk Wolda in Panama (see below in the type catalogue). Wolda was an entomologist and staff member at the Smithsonian Tropical Research Institute. There is no reference to this species in Brindle's notes from his analysis of the material (MMUE, Brindle archive, items 129, 138), from which he did, however, describe Pericomus ater as a new species.

5. 108 specimens of earwigs imported in foodstuffs or plant material form part of a reference collection (accession number F3231) initiated by W.D. Hincks (see section 'Walter Douglas Hincks' below). Five species in 4 genera are represented and include 89 specimens from East Africa, 14 from West Africa and 2 from Saudi Arabia, almost all found in imported bark or logs.

In summary, 835 species and subspecies are represented in the entire collection, approximately $44 \%$ of the Dermaptera world fauna. The number of species is comparable to those held at the British Natural History Museum (BMNH; now Natural History Museum), London (855 species). The number of specimens at MMUE is $75 \%$ that of BMNH, which holds 450 primary and 1200 secondary Dermaptera types (Natural History Museum 2015b). A complete type catalogue of Dermaptera at MMUE is provided below.

\section{Development of the Dermaptera collection}

Two curators of the Manchester Museum Entomology Department, Walter Douglas Hincks (1906-1961) and Alan Brindle (1915-2001), were chiefly responsible for developing the extent and geographical scope of the collection as it is today, both being the world authorities on the order in their time. Hincks was 
Table 2. Number of undetermined Dermaptera specimens in the Manchester Museum by geographical region.

\begin{tabular}{lcccc}
\hline & \multicolumn{4}{c}{ No. of specimens } \\
\cline { 2 - 5 } Region & Dried, mounted & Dried, papered & Spirit & Total \\
\hline Oriental & 464 & 1622 & 0 & 2086 \\
Afrotropical & 288 & 0 & 2 & 290 \\
Australasia & 191 & 0 & 0 & 191 \\
Neotropical & 300 & 185 & 470 & 955 \\
Palearctic & 46 & 202 & 5 & 253 \\
Nearctic & 2 & 0 & 0 & 2 \\
No data & 26 & 0 & 67 & 93 \\
& $\mathbf{1 3 1 7}$ & $\mathbf{2 0 0 9}$ & $\mathbf{5 4 4}$ & $\mathbf{3 8 7 0}$ \\
\hline
\end{tabular}

Assistant Keeper of Entomology at Manchester Museum from 1947 to 1961 (the title changed to Keeper in the 1950s) and Brindle was the Keeper of Entomology from 1961 to 1982.

The Museum was founded by the Manchester Society for the Promotion of Natural History in 1821. A history of the development of the museum is provided by Alberti (2009). An outline of the establishment of the Entomology Department is given by Johnson (1996) and Logunov (2010a), who pinpoint the department's beginnings to the promotion of John R. Hardy (1844-1921) to Senior Assistant Keeper and Curator of Entomology in January 1908. Prior to this, the insect collections inherited from the

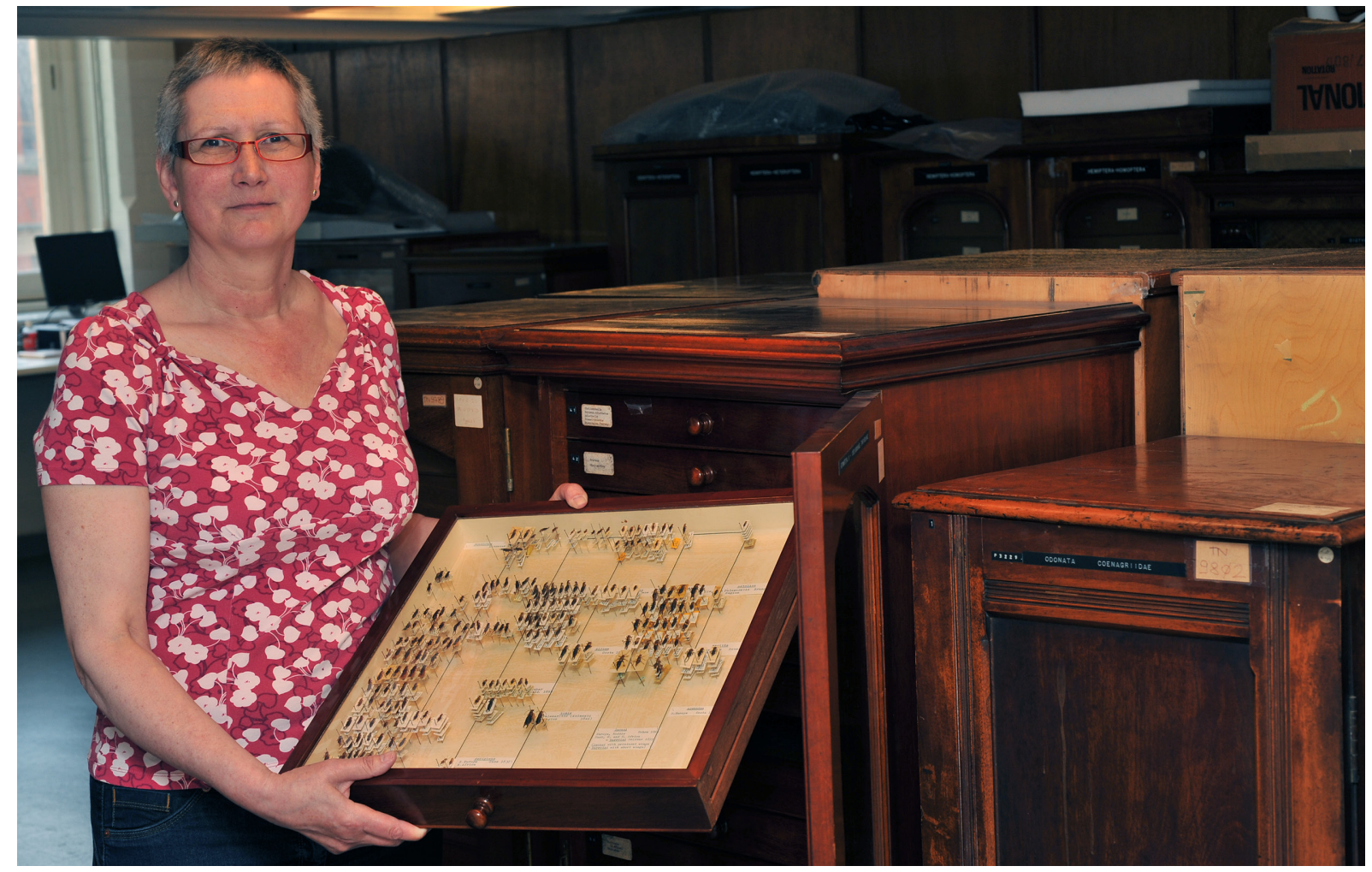

Fig. 1. The author and two of the three cabinets (drawers 21-60) housing the dry, mounted specimens in the worldwide Dermaptera collection, the Manchester Museum. 
Table 3. Number of Dermaptera specimens in the British Collection of the Manchester Museum, by species.

\begin{tabular}{lllc}
\hline Family & Genus & Species & No. of specimens \\
\hline Anisolabididae & Euborellia & annulipes & 32 \\
Forficulidae & Forficula & auricularia & 87 \\
Forficulidae & Forficula & lesnei & 9 \\
Forficulidae & Apterygida & media & 22 \\
Labiduridae & Labidura & riparia & 1 \\
Spongiphoridae & Labia & minor & 95 \\
Spongiphoridae & Marava & arachidis & 4 \\
Undetermined & - & - & 1 \\
Total & & & $\mathbf{2 5 1}$ \\
\hline
\end{tabular}

${ }^{1}$ Collected in France.

Manchester Natural History Society were in such a poor state that it was considered necessary to start them again from scratch (Report 1890-94).

In 1951 Hincks wrote:

“... it has been stated of various orders or groups of insects that they are 'neglected'. To none is the epithet more truly applicable than to the order Dermaptera or Earwigs, the serious students of which, at any one time, could be counted on the fingers of one hand" (Hincks 1951a: 1).

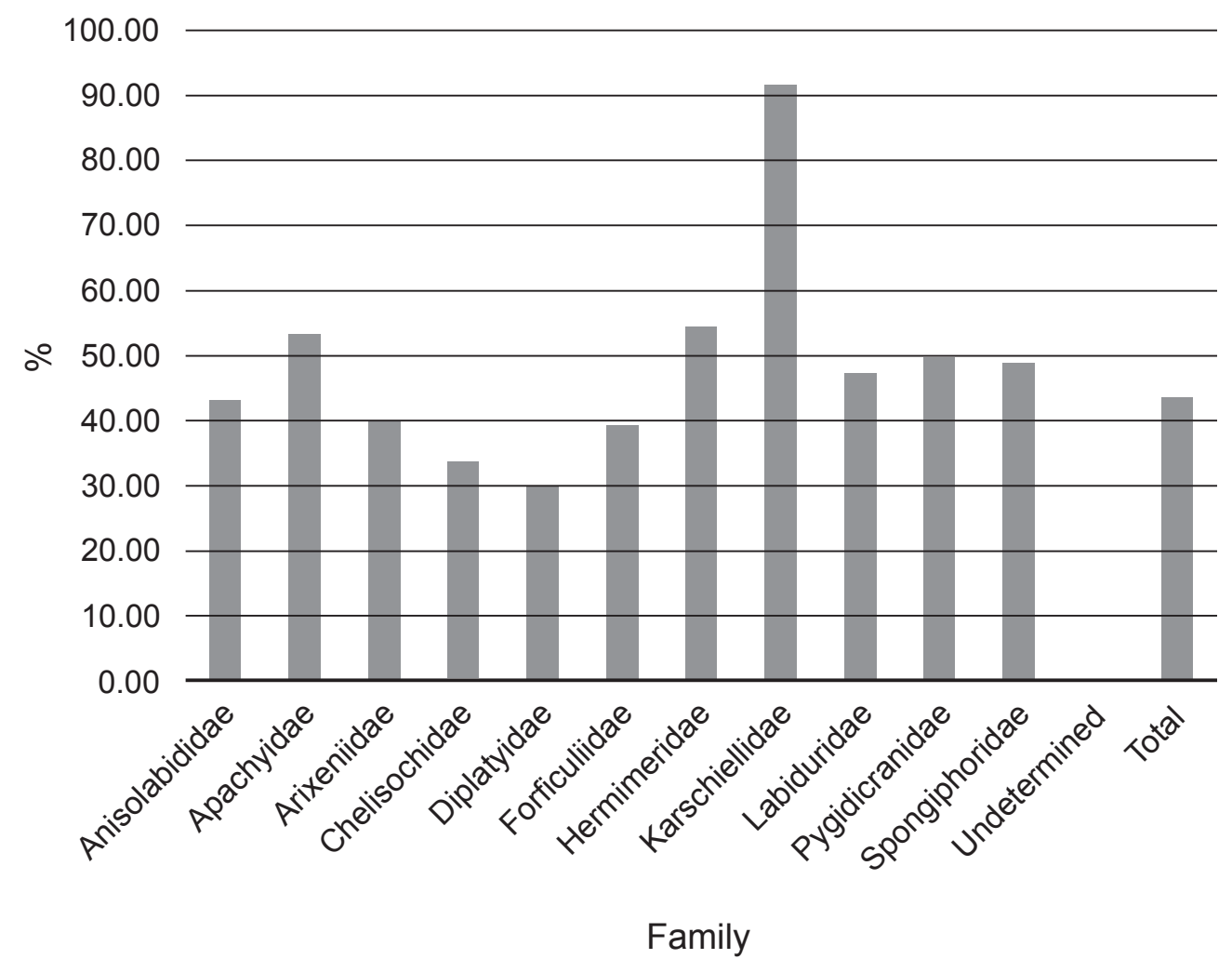

Fig. 2. A graph showing \% of World Dermaptera represented at the Manchester Museum, by family. 
It is not surprising then that from the first entry in the Entomology Department's Accession Register (Volume 'F') in 1889 by Hardy until the 1950s, with one exception, there is no specific mention of Dermaptera acquisitions in the Museum's Annual Reports or in the Accession Register. The exception is three specimens of the parasitic Hemimerus talpoides Walker, 1871 from the Gold Coast, West Africa, donated by Prof. Hickson in 1919 (accession no. F1809; see Parasitic Earwigs below). That is not to say earwigs were not acquired. In the early part of the century it is likely they were included in the unspecified 'Insects' and possibly 'Orthoptera' received from around the world, and certainly from the time of Hincks in the late 40s onwards, Dermaptera started to flood into the Department. The acquisitions were not, however, entered into the Accession Register as a matter of routine.

Much research would be necessary to identify the sometimes tortuous routes by which many of the early specimens came into the collection; as described below, many were acquired through exchange, particularly by Hincks and Brindle. The earliest dated specimen in the Dermaptera collection is an Apachyus chartaceus (Haan, 1842) dated 1878 (Fig. 5). It was collected during a Dutch scientific expedition to Sumatra 1877-1879 under the supervision of the President of the Royal Dutch Geographical Society, Prof. P.J. Veth (Wentholt \& Wilschut 2003). Dermaptera material from the expedition was sent to Auguste de Bormans of Belgium for identification (Veth 1892), considered 'the only and final

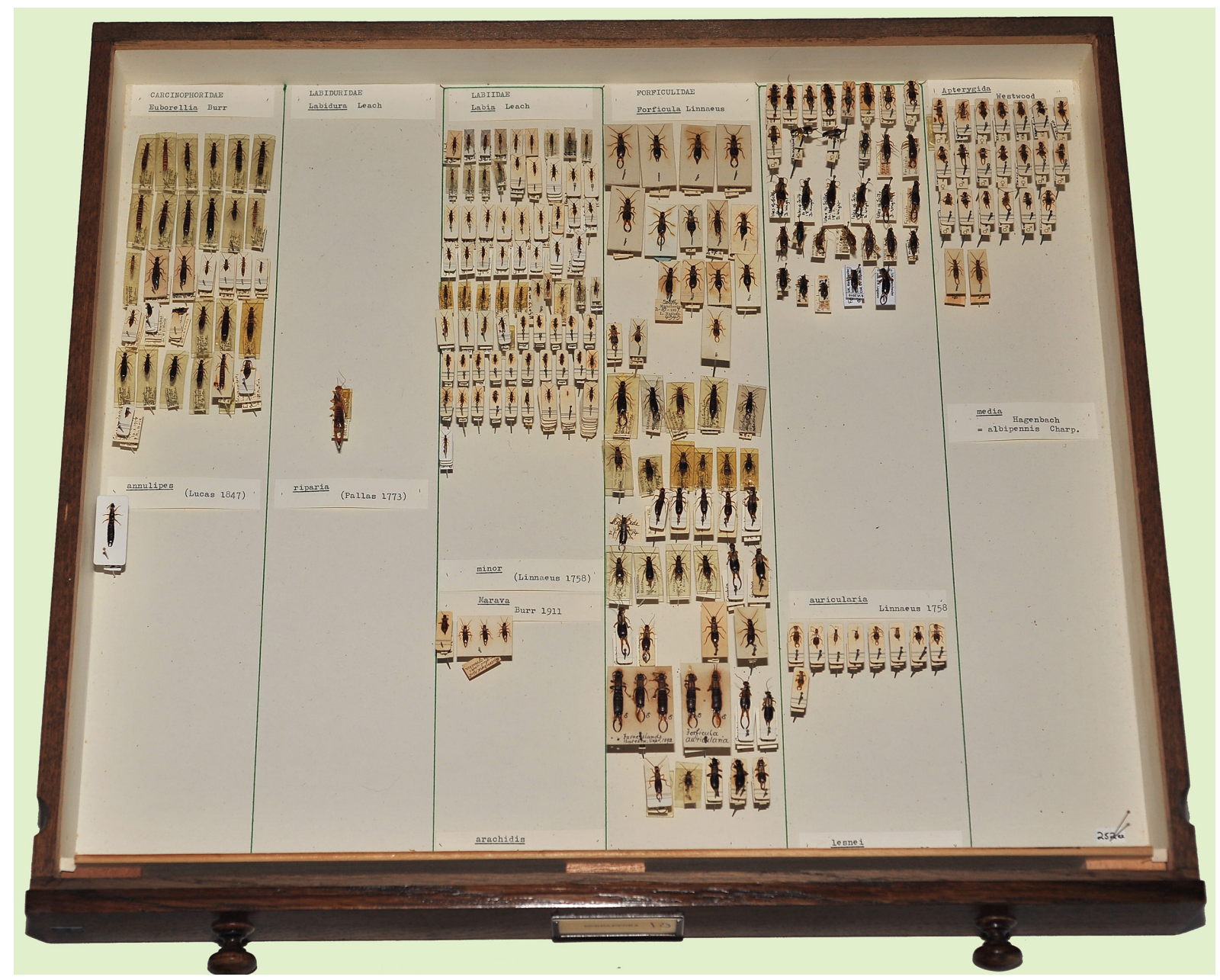

Fig. 3. A drawer of the British Dermaptera collection, the Manchester Museum. 
authority' on Dermaptera at the time (Burr 1901: 85). De Bormans' collection was acquired by Malcolm Burr (see below) and later sold to BMNH in the early 1900s (letter from Burr to Hincks, MMUE, Hincks archive, item 54; Natural History Museum 2015a).

Other specimens dating from the end of the 19th century were collected by Leonardo Fea (1852-1903) in Burma (now Myanmar), and by Hans Frühstorfer (1866-1922) in Java in 1893 and Lombok, Lesser Sunda Islands in 1896. Fea was an Italian zoologist who worked at the Genoa Natural History Museum and made several foreign trips to collect specimens, exploring extensively in Burma in the late 1880s (Gorman 1892). Frühstorfer was described as ".... purely entomological and systematic genius, the like of which we have never met before and are not likely to meet again" (Martin 1922: 97). In Lombok he met and worked with William Doherty (1857-1901), "Prince of collectors" (Martin 1922: 99), who collected MMUE's specimen of Cordax armatus (de Haan, 1842) in Macassar, Indonesia in 1896.

In 1919, Harry Britten (1870-1954) was appointed as Hardy's successor as Assistant Keeper at Manchester Museum. According to Hincks, Britten was "the greatest British entomologist since the days of Curtis and Stephens" (Hincks 1954a: 225). He particularly added to the extent of almost all orders of British insects in the collection and improved their arrangement (Report 1923-24). He and Hincks each provided 50-60 British earwig specimens and between them collected almost 50\% of the specimens in the British collection. Britten's specimens are almost entirely from Lancashire and Cheshire, collected between 1919 and 1948, overlapping Hincks' which date from 1932 to 1951. The earliest dated specimens are Forficula auricularia Linnaeus, 1758 collected in 1892 in the Farne Islands, the label only identifying the collector as 'Bateson'. The most recent specimen (also F. auricularia) was collected in 2013 at Manchester University by the current Curator of Arthropods, Dmitri Logunov.

There is little information about the contribution to the Dermaptera collection of Geoffrey Kerrich (19092003), who followed Britten as Assistant Keeper in 1937. He was seconded away from the museum to other duties a year after the start of the war and only returned half-time in 1943. His particular interest was parasitoid Hymenoptera (Noyes 2003). The Annual Report of 1940-41 notes that he began to build up "... a collection of immature forms in fluid preservative ...", although it is not specified whether this included any earwigs (Report 1940-41: 7).
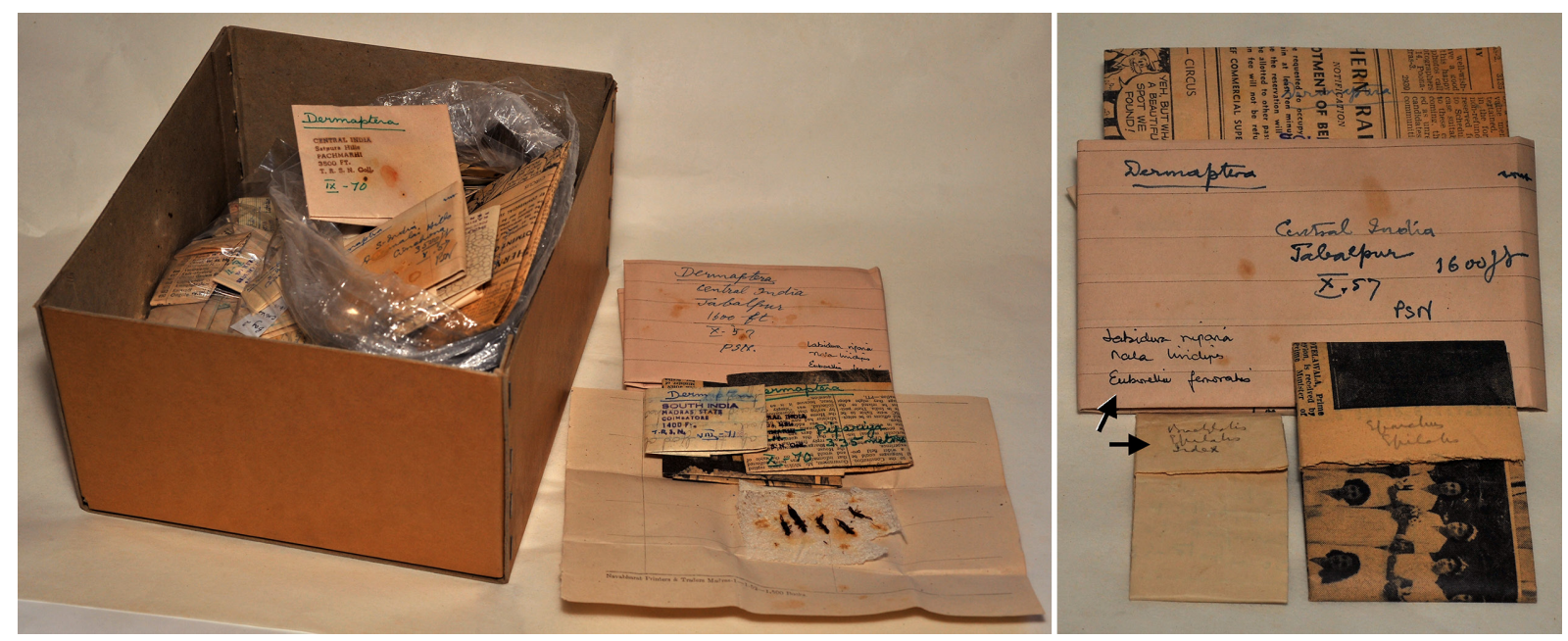

Fig. 4. Papered material from India collected by P.S. Nathan and T.R.S. Nathan between 1953 and 1972. Arrows indicate Brindle's identifications (see comments in the text under 'Alan Brindle'). 


\section{Walter Douglas Hincks (1906-1961)}

The development of the Dermaptera collection really took off when Hincks (Fig. 6) was appointed Assistant Keeper in August 1947. When he took over, "with the aid of his assistant Stanley Shaw, and later Alan Brindle, the rearrangement and combination of these collections was commenced with the object of making the Museum's Entomology Department the finest reference and study centre in the North" (Kloet 1961: 183). Hincks developed his interest in entomology as a member of the Leeds Naturalists' Club while studying to qualify as a pharmacist (Thimann 1961). He went on to work for a large firm of manufacturing chemists. Kloet (1961) describes how Hincks developed his expertise first as

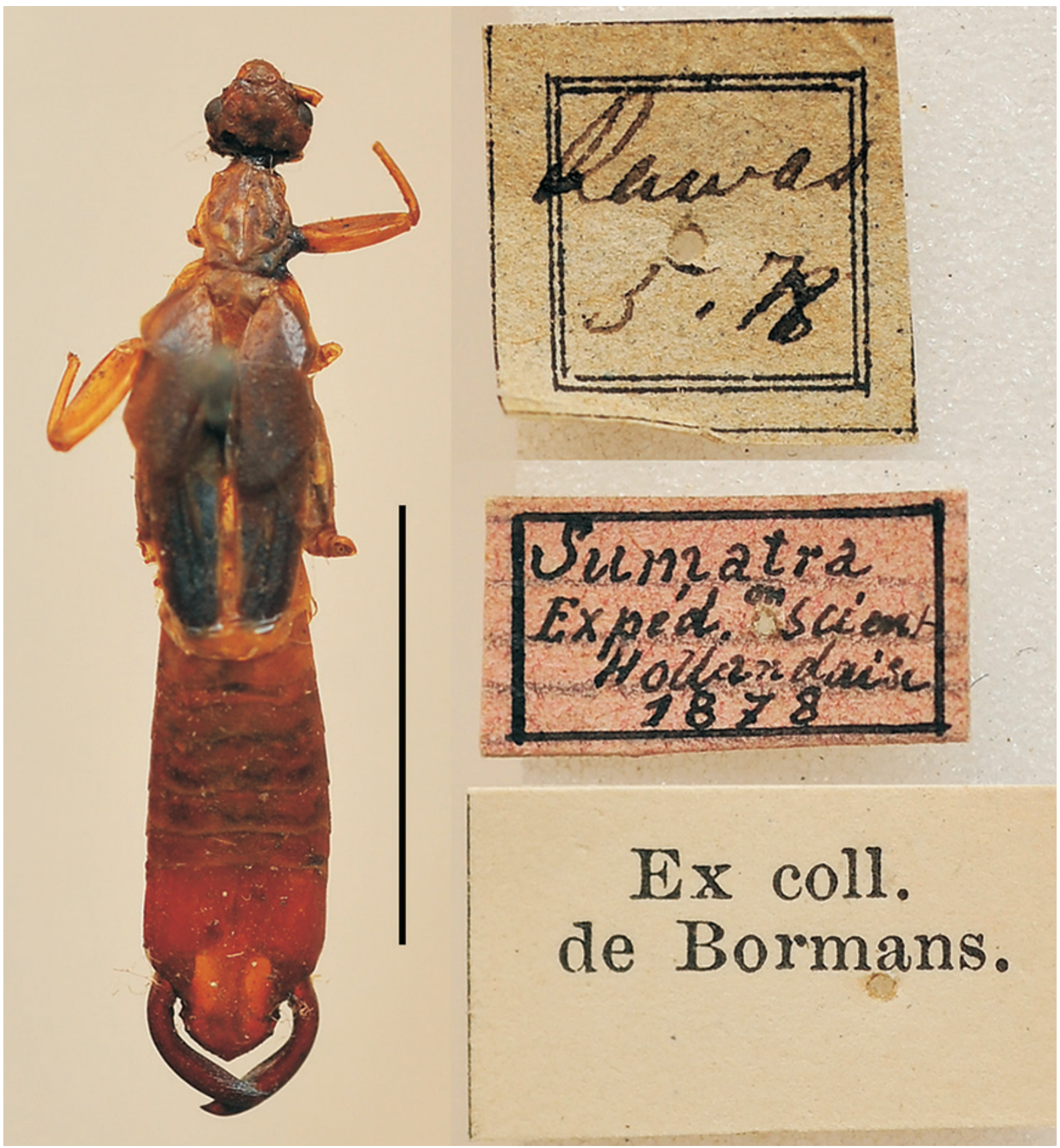

Fig. 5. The collection's earliest dated specimen, Apachyus chartaceus (Haan, 1842), dated 1878, the Manchester Museum. Scale bar $=1 \mathrm{~cm}$. 
a world authority on Passalidae, with his friend John Dibb, and then extended his studies to Orthoptera, Chironomidae, Diptera, Cassidinae and parasitic Hymenoptera. At the same time he built up a large private collection and during his life "... played a most important role in the organisations devoted to the promotion of Entomological knowledge" (Kloet 1961: 182). He is well known for the comprehensive 24-volume Check List of British Insects compiled in collaboration with Kloet, published in 1945 (Kitchen 1962). This publication formed the structure for the future layout of the British collections at the Manchester Museum (Johnson 1996).

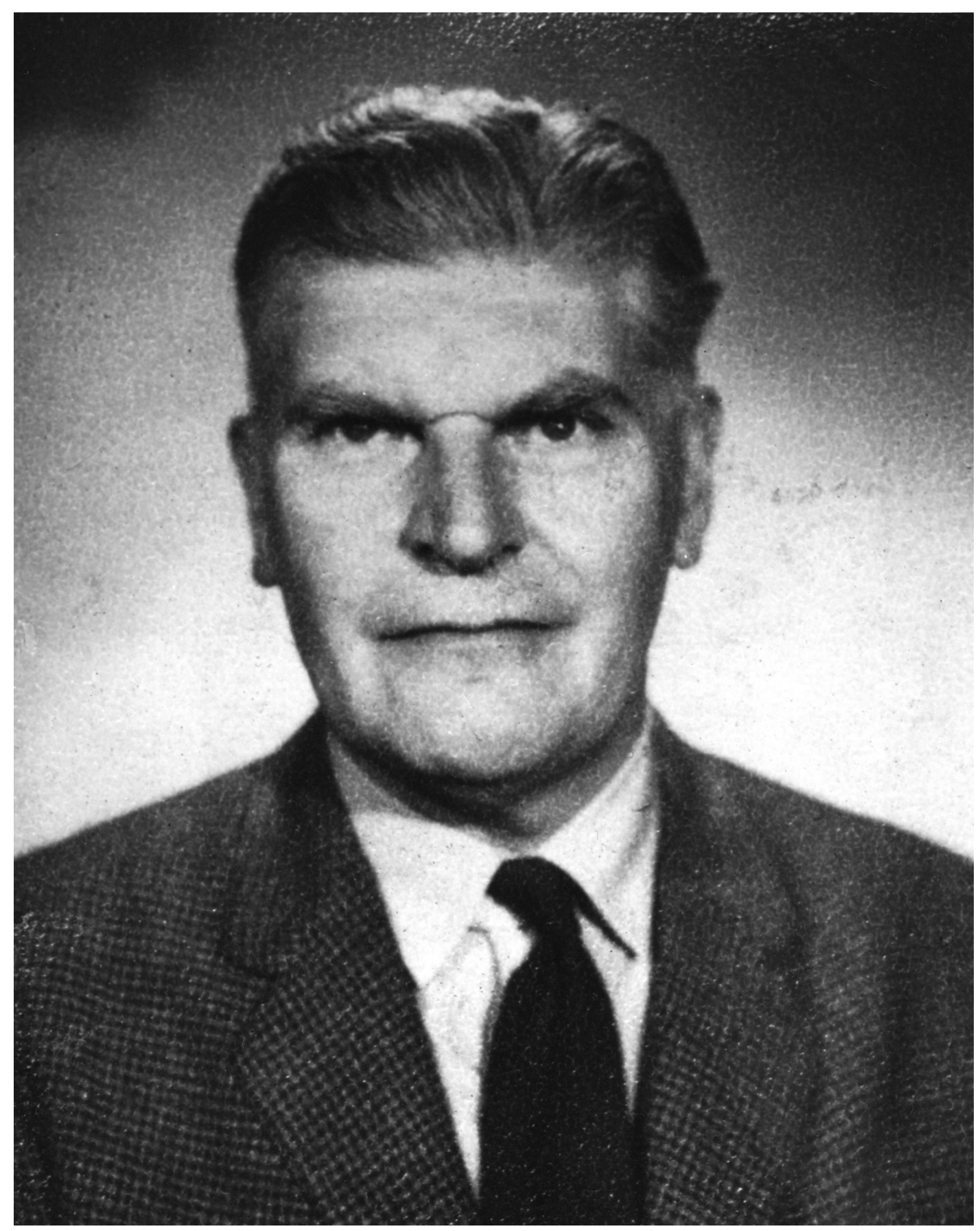

Fig. 6. Walter Douglas Hincks (1906-1961), Keeper of Entomology at the Manchester Museum (19471961), photographed in the late 1950s. 
Hincks began to focus on the study of Dermaptera in the early 1930s and was encouraged and supported by Dr. Malcolm Burr (1878-1954), a pioneer in the field who was originally a specialist in European Orthoptera but became an accepted authority on Dermaptera (Hincks 1954d). Burr trained as a geologist, but was also a journalist, author and in his later years, an English professor at Istanbul University (Harvey et al. 1996). The two men kept up a sustained correspondence. 34 letters from the period January 1933 to October 1951 are held in the Museum's entomological archive. In January 1933, Burr wrote, "I am so delighted that some one [sic], esp. an Englishman, has come forward to carry on the work [on Dermaptera] where I gave up" (MMUE, Hincks archive, item 52) and writing to Hincks from Istanbul in 1950, Burr wrote, "I should like to see you chuck the beetles and go all out on earwigs" (MMUE, Hincks archive, item 42). Hincks did make earwigs one of his special areas of study, becoming a major contributor to the development of the taxonomy of the order. Kloet said of him:

"... he realised that here lay the greatest scope for research and a task that would test to the utmost his skill as a systematist and taxonomist and exercise his talent for the investigation of nomenclature and synonymy. It is rare, indeed, to find a man capable of all these approaches, yet Hincks was undoubtedly master of them all' (Kloet 1961: 182).

One of the reasons Hincks gave for the lack of progress with Dermaptera taxonomy was that, until Burr, no-one had enough material to develop a sound classification of the order (Hincks 1951a). In February 1933, Burr exhorted Hincks, “... the chief thing is to get a representative set of as many genera as poss. You will find my coll. in the B.M. the completest in existence still" (MMUE, Hincks archive, item 54). This was backed up by James Rehn (1881-1965), Acting Curator at the Academy of Natural Sciences of Philadelphia, who wrote on 3 Mar. 1933:

"It may be possible for me in the not distant future to send material of a few species of earwigs which are included in our duplicate series, but my experience has been much the same as Dr. Burr's, in that earwigs rarely come to the student in sufficient series to more than supply his working representation" (MMUE, Hincks archive, item 56).

Hincks therefore worked assiduously to build up a comprehensive earwig collection and achieved this by several means. To widen the geographic range of the collection, he requested or encouraged others to request collectors to provide specimens from particular areas, and at the same time arranged the purchase of specimens directly from collectors. Correspondence in the Hincks archive, retained at the Manchester Museum, evidences his network of contacts with those at the forefront in the study of Dermaptera such as Friedrich Zacher (1884-1961), J.A. Rehn, Alfredo Borelli (1858-1943), Carlo Menozzi (1892-1943) (Gilbert 1977) and Grigory Bey-Bienko (1903-1971) (Rentz 2014; MMUE, Hincks archive, items 48, $72,56,58,85,90,387)$. Appendix I provides a list of Hincks' correspondence specifically relating to Dermaptera. In 1938 he advertised in the publication Insektenbörse to buy Dermaptera material. This resulted in the purchase of 370 earwigs from Java (supplied by H. Lucht and M.E. Walsh) and 270-80 from Paraguay (supplied by F.H. Schade) (MMUE, Hincks archive, items 45-7, 104-6, 124).

From all this material, he determined and described new species and this taxonomic work led to scholarly publications. His philosophy was that the department “... should carry out taxonomic and biogeographical research at the international level, based upon the collections of overseas museums as well as its own" (Johnson 1996: 205). In his first busy year as Assistant Keeper, the Annual Report records that in addition to all his other curatorial duties, "A monograph of the Earwigs of the world has been initiated and materials accumulated for the purpose." (Report 1947-48: 11).

Part I of his Systematic Monograph of the Dermaptera of the World based on material at BMNH was published in 1955 and the second (and last) of the intended six volumes was published in 1959. Fig. 7 shows an example of Hincks' original monograph illustrations and Fig. 8 shows a page from his 
'Typomap of Africa'. His contribution to the London Royal Entomological Society's Handbooks for the identification of British insects on Dermaptera and Orthoptera was published in 1956. By the time of his death, he had published more than three hundred papers and notes in international, national and regional journals (Johnson 1996), including more than 70 on Dermaptera.

Hincks' expertise led to increased requests for identification of material from Public Health Departments, amateur naturalists and other inquirers and, particularly, to specialised identification work for various British and overseas museums and institutions. The accepted custom of retaining part of the material for the Museum led to a rapid increase in the size and scope of the collection (Reports 1953-58). Some enquiries arose from the accidental importation and discovery of insects in timber and foodstuffs, and were numerous enough that Hincks saw the need to create a reference collection of such insects. This

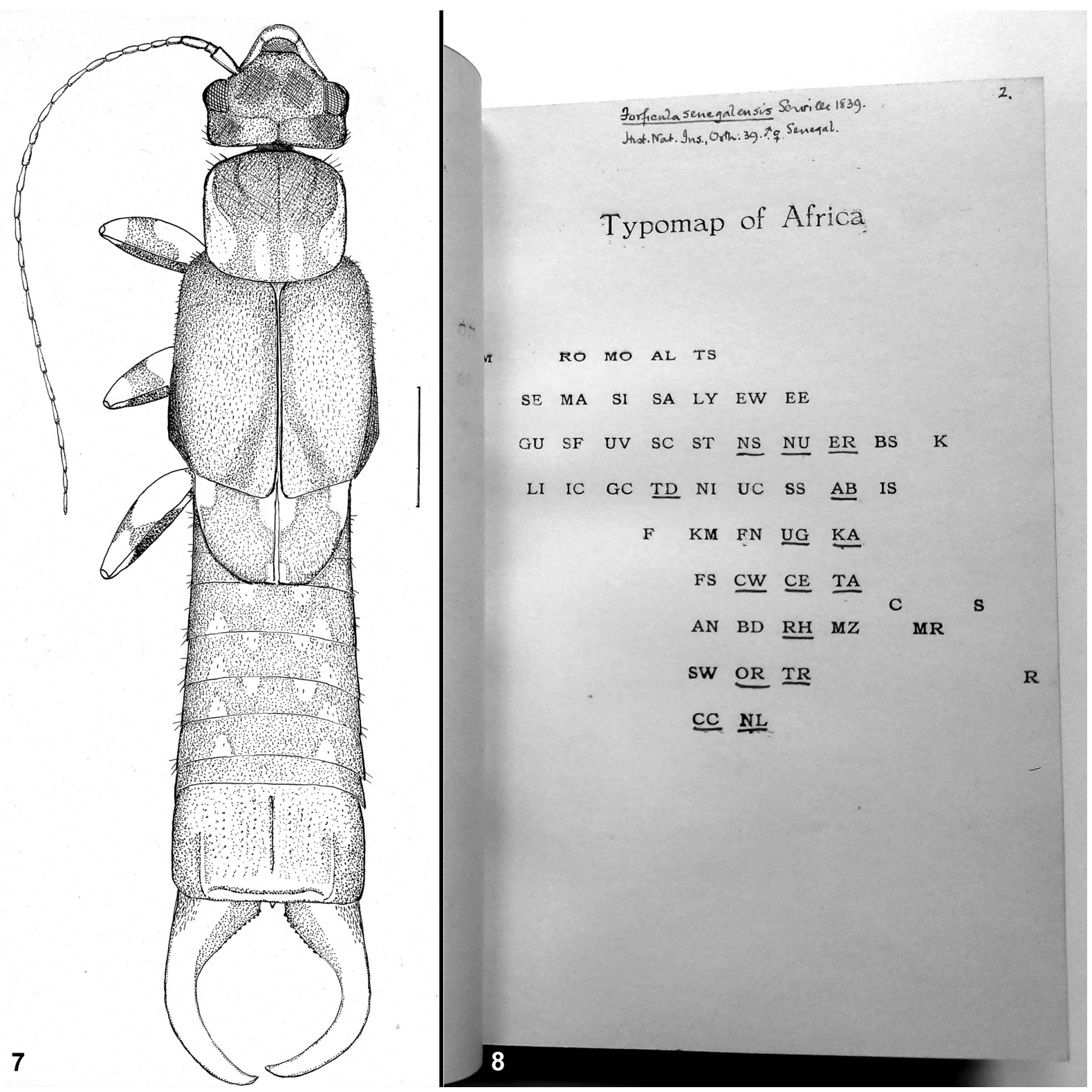

Figs 7-8. Two items from Hincks' archive retained at the Manchester Museum. 7. An illustration of Pyragra paraguayensis Borelli, 1904 (đ̋) by Hincks for his Dermaptera monograph (Hincks 1959: 195) (item 597). 8. A page from Hincks' 'Typomap of Africa' (item 15). 
developed from its inception in 1951 (Report 1950-51) to contain 463 identified insects in 7 orders in 2010 (Logunov 2010a); however, virtually all the Dermaptera material in this collection was collected between 1948 and 1955 and arranged by Hincks (See 'Description of the Dermaptera collection' above).

In addition, material was collected on the many field trips, surveys and courses directed by or attended by Hincks, Shaw, and later, Brindle, both in Britain and abroad, although there is a comment in the Annual Report for 1956-57 (p. 10) that “... collecting and other field activities have been purposely curtailed this year because of the vast number of unidentified specimens which have accumulated in the department". Fig. 9 shows Hincks and Brindle during one of the Insect Surveys at Freshfield, Lancashire. Six specimens in the British earwig collection resulted from these surveys. Shaw left the department in May 1956 and Alan Brindle, "an entomologist of considerable experience", joined Hincks as his assistant in 1958 (Report 1958-9: 8).

By the time of his death in 1961, Hincks had become a world authority on Dermaptera, Passalidae and Chrysomelidae and improved the Manchester collection such that it was "... now the most important collection in the country after the British Museum and the Hope Department, Oxford, its types and figured specimens running into many thousands" (Report 1960-61: 1). Hincks' personal collection of World Dermaptera was purchased by the Museum in 1961 (accession no. F2440) along with his Passalidae and Chrysomelidae collections. The Dermaptera collection was second only to the British Museum collections and at that time included some 200 'types and paratypes', i.e., $27 \%$ of the type material held now. It was housed in twenty cabinet drawers "already arranged", the collections being “... beautifully documented and of great importance for study" (Report 1961-2: 14, 8, 2).

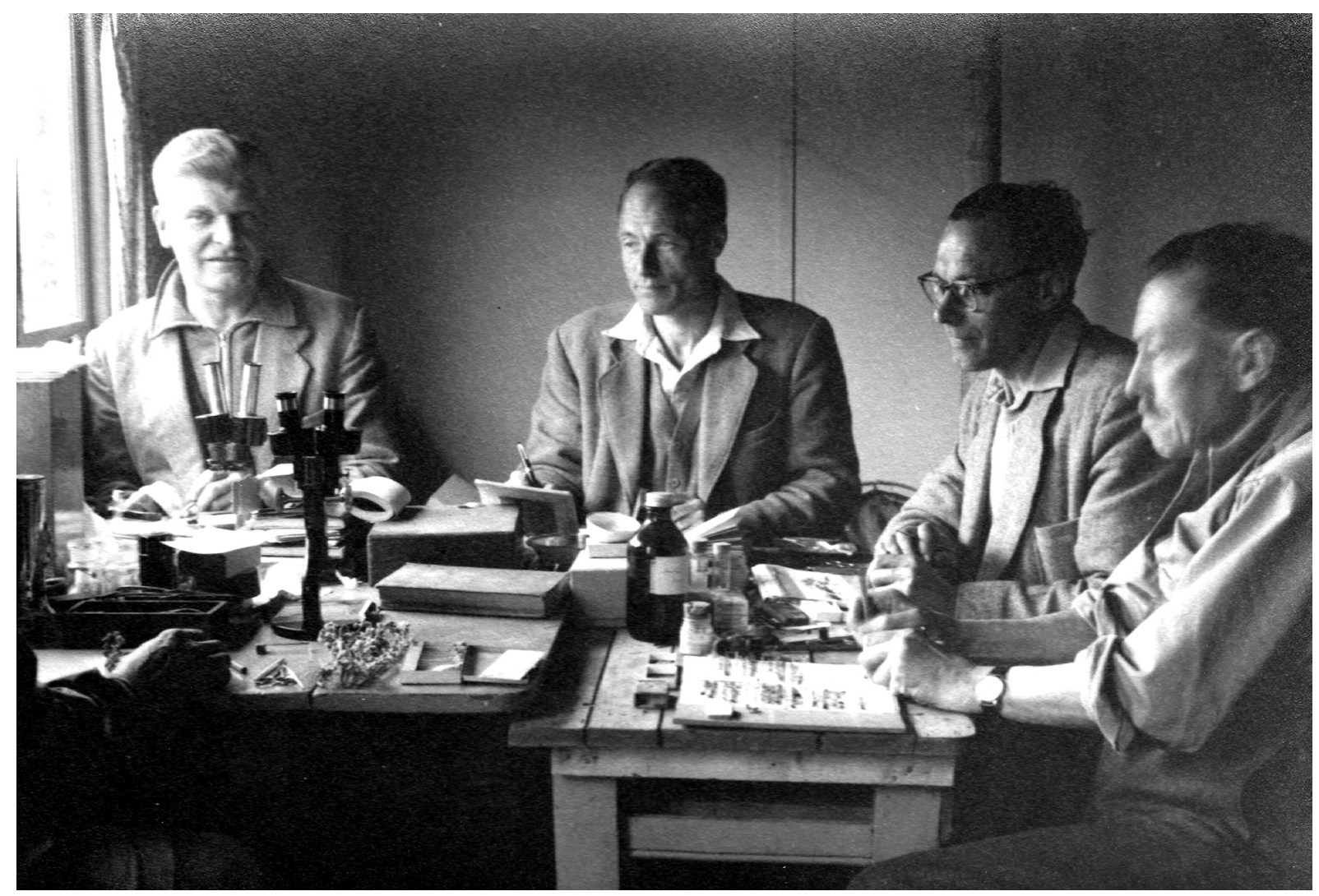

Fig. 9. Freshfield Insect Survey, June 1959. Left to right: W.D. Hincks, M.W.R. de Vere Graham, A. Brindle and C.H. Wallace Pugh (MMUE archive). 


\section{Alan Brindle (1915-2001)}

Following Hincks' death, Alan Brindle (Fig. 10) was promoted to Keeper of Entomology, a post he held until his retirement in 1982. He had started work in a Lancashire woollen mill and at the same time attended night school to learn German, French and Russian. During the war he worked in intelligence in Pakistan and Bangladesh and after the war taught secondary biology. He developed an interest in Lepidoptera in his teens, collecting around Pendle Hill, and also took the opportunity to collect snake skins, butterflies and other insects during his war postings (Johnson 2003). He was elected Fellow of the Royal Entomological Society in 1941 and awarded an MSc by the University of Manchester for his work on caddisfly biology in 1968 (Report 2000-01).

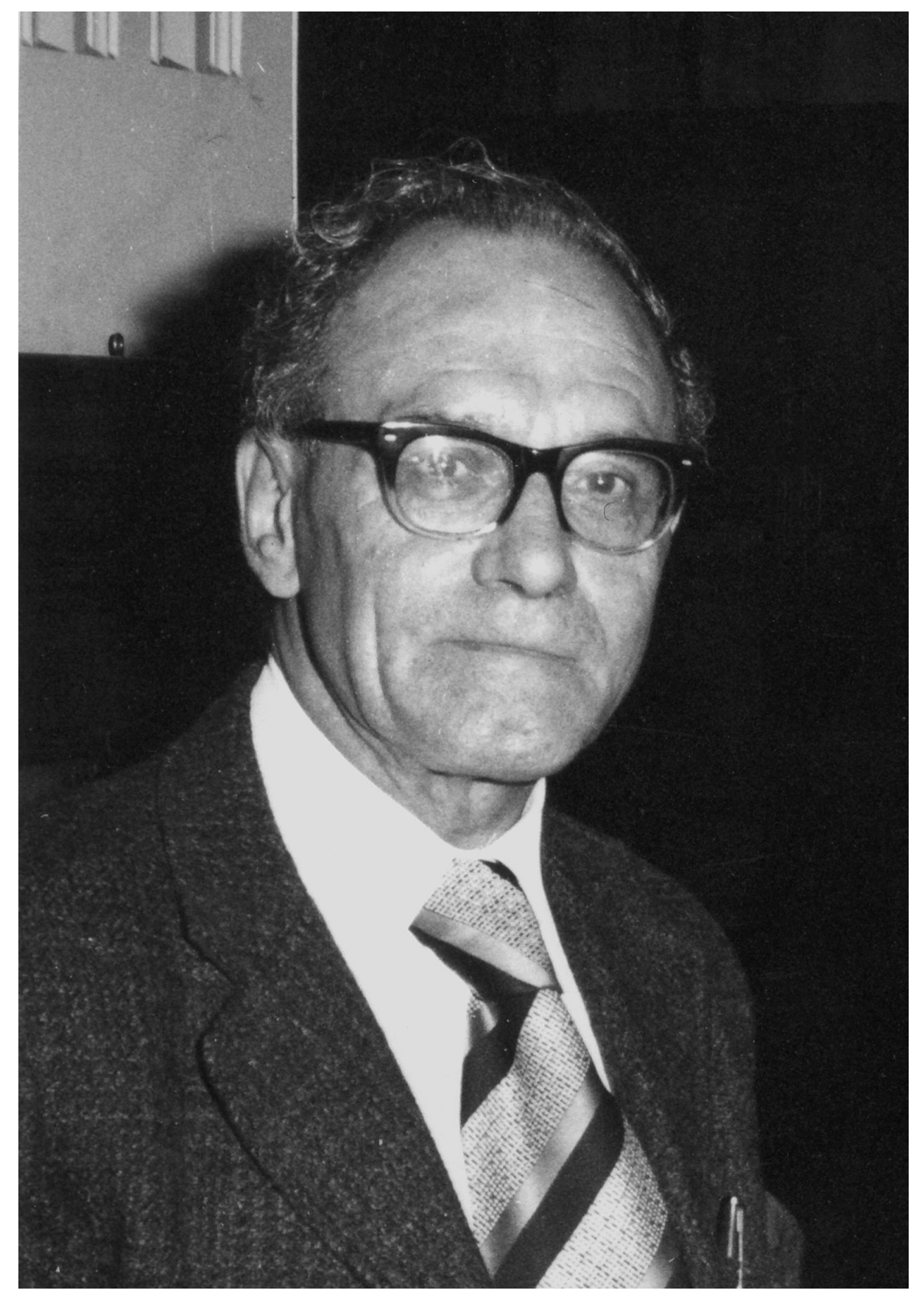

Fig. 10. Alan Brindle (1915-2001), Keeper of Entomology at the Manchester Museum (1961-1982), photographed in the mid 1980s. 
According to Johnson (1996), Brindle subscribed to Hincks' philosophy that entomologists should become taxonomic specialists in an order or family, within the international scientific community. After Hincks' death, Brindle took up and continued Hincks' work on Dermaptera, publishing his first paper in 1964 with G. Friese on Albanian earwigs. Prior to this he had already published over 60 papers, the majority of them on Trichoptera and the larvae of British Tipulinae and British Diptera. Dermaptera took over and between then and 1994 he published descriptions of a staggering "351 new taxa (321 species, 11 subspecies, 15 genera and 4 subfamilies) in 126 papers" (Johnson C. 2003: 57). His great work, "The Dermaptera of Africa', was published in two parts in 1973 and 1978 by the Musée Royal de l'Afrique Centrale, Tervuren, Belgium.

Large numbers of Dermaptera continued to be received for identification from museums and scientific institutions around the world. Each year hundreds of specimens were identified by Brindle and returned and, according to custom, it was allowed that some specimens be retained at MMUE. Most of these were not recorded in the accession register but this sort of work was a regular feature in the Annual Reports, a typical one from 1965-66 recounting that of the earwigs received from various institutions, most were new to the collection and "... a good proportion are species new to science" (Report 1965-66: 14). In 1973 the workload was so heavy that Brindle had to return some Cassidinae unidentified to Dr Richard zur Strassen of the Research Institute Senckenberg, Frankfurt as "I am working on Dermaptera and do not see any chance of working on the Cassidinae for some years to come" (MMUE, Brindle archive, item 174). As another example, Brindle was asked to examine approximately 5000 specimens from Panama, from a collection organised by the Smithsonian Tropical Research Institute. From this material he described 14 new species (Brindle 1981, 1982a, 1988). His notes state that 802 specimens of 31 species from this material were retained at MMUE (MMUE, Brindle archive, item 138). The only earwig in the collection mounted with wings open was part of this consignment (Fig. 11).

Like Hincks, Brindle also sought out specimens from specific areas. Correspondence between Brindle, P.S. Nathan and his daughter-in-law T.R.S. Nathan shows that material from South India was requested and received from before November 1964 to at least September 1972 (MMUE, Brindle Archive, items

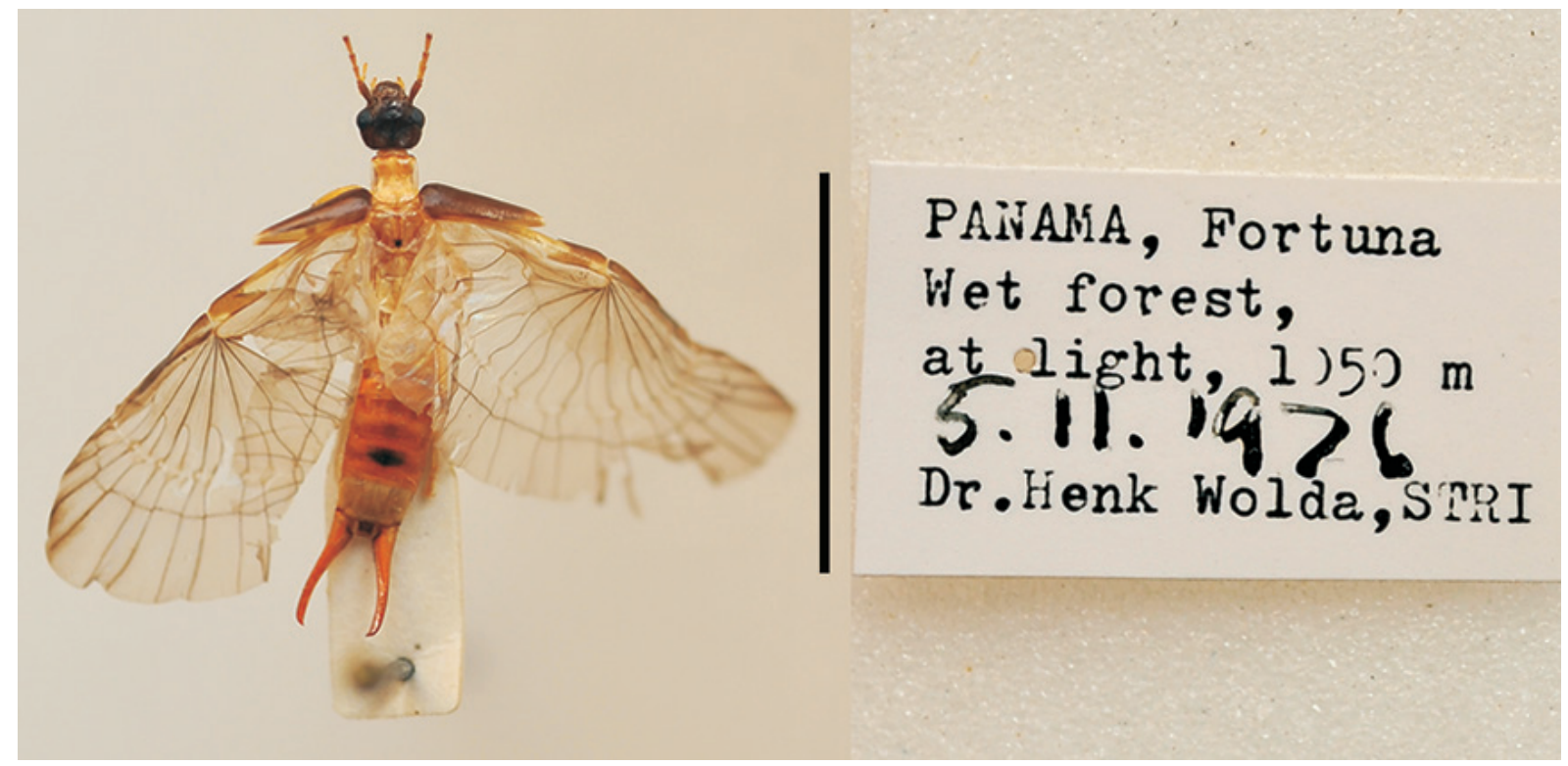

Fig. 11. The only specimen at MMUE mounted with wings unfurled, Purex remotus (Burr, 1899) from Panama, the Manchester Museum. Scale bar $=1 \mathrm{~cm}$. 
215-234). There are several hundreds of Nathan contributions mounted, determined and incorporated into the collection, with 1600 remaining as papered material. Brindle's handwritten notes on some packets identify genus and in some cases species, the majority of these packets containing mixed species or genera. Both Hincks and Brindle named species for Nathan - Diplatys nathani Hincks, 1960 and Gonolabidura nathani Brindle, 1965. Hincks acknowledged that Nathan's “... expert field work has provided many Museums with important insect materials from India" (Hincks 1960a: 15) and Brindle that his "... untiring efforts in collecting specimens have considerably increased our knowledge of the Dermaptera of India" (Brindle 1965: 594).

During 1979-80, Dr Seiroku Sakai (1924-2004) visited the Manchester collection on his travels around the world gathering original descriptions of new Dermaptera species and photographing existing type specimens for his encyclopaedic 'Dermapterorum Catalogus: A Basic Survey for Integrated Taxonomy of the Dermaptera of the World' (Masaki 2006). He met with Brindle and with Prof. E.J. Popham of Manchester University to discuss classification (Report 1979-80: 15). They kept in contact and the Brindle Archive holds Brindle's working copy of Sakai's 'A new proposed classification of the Dermaptera with special reference to the checklist of the Dermaptera of the world' (Sakai 1982), with further corrections and editions, as well as reprints of Sakai's papers which he sent to Brindle.

Johnson (1996) estimated that during Brindle's period as Keeper, the Dermaptera collection doubled in size to around 10,000 specimens. The great numbers of specimens coming in from such identification requests led to the re-curation of the Dermaptera in three cabinets with sixty drawers (Fig. 1). The first part of the newly reorganised collection was arranged according to Hincks' 'Systematic Monograph of the World Dermaptera' and the rest of the collection was arranged according to "... revisions of certain groups of the earwigs by the Keeper" (Report 1967-68: 10). The storage of the Dermaptera spirit collection today is virtually unchanged since Brindle re-curated it during 1972-3 (Report 1972-3).

Brindle retired in 1982, a much respected scientist who had overseen significant development of the Museum's entomological collections and gained a "... high reputation in the fields of classification of world Dermaptera (Earwigs) and taxonomy of Diptera (fly) larvae". He was remembered as a "... kindly, modest and thoughtful man, who wore his knowledge lightly and was always ready to help and encourage others" (Report 1981-82: 17).

Since his time, only around 30 specimens have been added to the collection, the most recent being three specimens collected by Dmitri Logunov in Peru in 2014.

\section{The Entomological Archive}

In addition to a library of specialist books and reprints, the Manchester Museum's Entomology department holds thousands of documents relating to the Keepers and associated collectors, organised into 34 collections. The following extracts summarise material relating to Brindle and Hincks (after Logunov 2010a: 40):

Brindle, Alan 599 [items]. In five boxes. Correspondence with H.G. Briggs, I.M. Robson, H. Robertson, T. Cekalovic, T.R.S. Nathan, G. Kerrich, and others (1933-84, 165 items); 'Insect records' (10 volumes, 1930-82); species records of aquatic insects in the museum collection, plus locality list; reprint collection (346 items); index records; typed and handwritten lists of insect species; Lepidoptera records; various keys; address books; visitor book; Diptera larvae catalogue; etc.

Hincks, Walter D. 611 [items]. In five boxes. Original figures for Dermaptera monograph (383 cards); published papers by Hincks (94 items); reprints Hymenoptera Parasitica J. Lancs. \& Chesh. Ent. Soc., $133 \& 134$ (2009 \& 2010) 41 and others; address and field notebooks (26 items); notes on Dermaptera 
and manuscripts on Passalidae; diaries (1948-49, 1956); correspondence with F. Spaeth, J.T. Salmon, M. Burr, W. Junk, and others (453 items).

Hincks' and Brindle's correspondence relating particularly to the Dermaptera collection is listed in Appendices I and II. Due to the particular interest of these two curators, the entomology department has a comprehensive library of their articles and reprints relating to Dermaptera as well as those of other authors.

\section{Notable earwig specimens in the MMUE collection}

\section{Giant Earwig, Labidura herculeana (Fabricius, 1798)}

The collection contains two specimens (one male, one female) of the iconic Giant Earwig Labidura herculeana (Fabricius, 1798) (accession nos. F3233.1871 (male) and F3233.1872 (female)) (Fig. 12).

The species is notable for being the largest known earwig, with one specimen in the Tervuren collections measuring $78 \mathrm{~mm}$ long and some forceps remains suggesting even larger specimens existed (Brindle 1970c). The MMUE specimens were amongst 40 collected during expeditions in 1965-66 and 1967 by the Musée Royal de l'Afrique Centrale (RMAC), Tervuren, Belgium. The species is also sadly notable for being recently assessed extinct. Endemic to the island of St. Helena in the South Atlantic Ocean, several unsuccessful searches have been made for them since and the species was assessed extinct in August 2014, the last living specimen having been observed in May 1967 (Pryce \& White 2014).

Downgraded to a subspecies of L. riparia by Kirby in 1904, in 1970 Brindle reinstated L. herculeana to a full species (Brindle 1970c) after examining the 40 RMAC specimens. At the time he suggested that "...the fact that this species is only known from this small area of St. Helena, and is not known from anywhere else in the world, must give rise to some concern about its future status" (Brindle 1970c: 215). Pryce \& White (2014) record that its decline may be due to the removal, for construction, of surface stones under which the earwigs would burrow, as well as increased predation by rodents and possibly invertebrates, including spiders and the centipede Scolopendra morsitans Linnaeus, 1758.

Brindle (1970c) also synonymised L. loveridgei Zeuner, 1962 with L. herculeana (Fabricius, 1798). $L$. loveridgei had been described from forceps remains and named for the zoologist Arthur Loveridge (1891-1980) who had moved to live on the island in 1957. Hincks had seen the forceps and also assigned

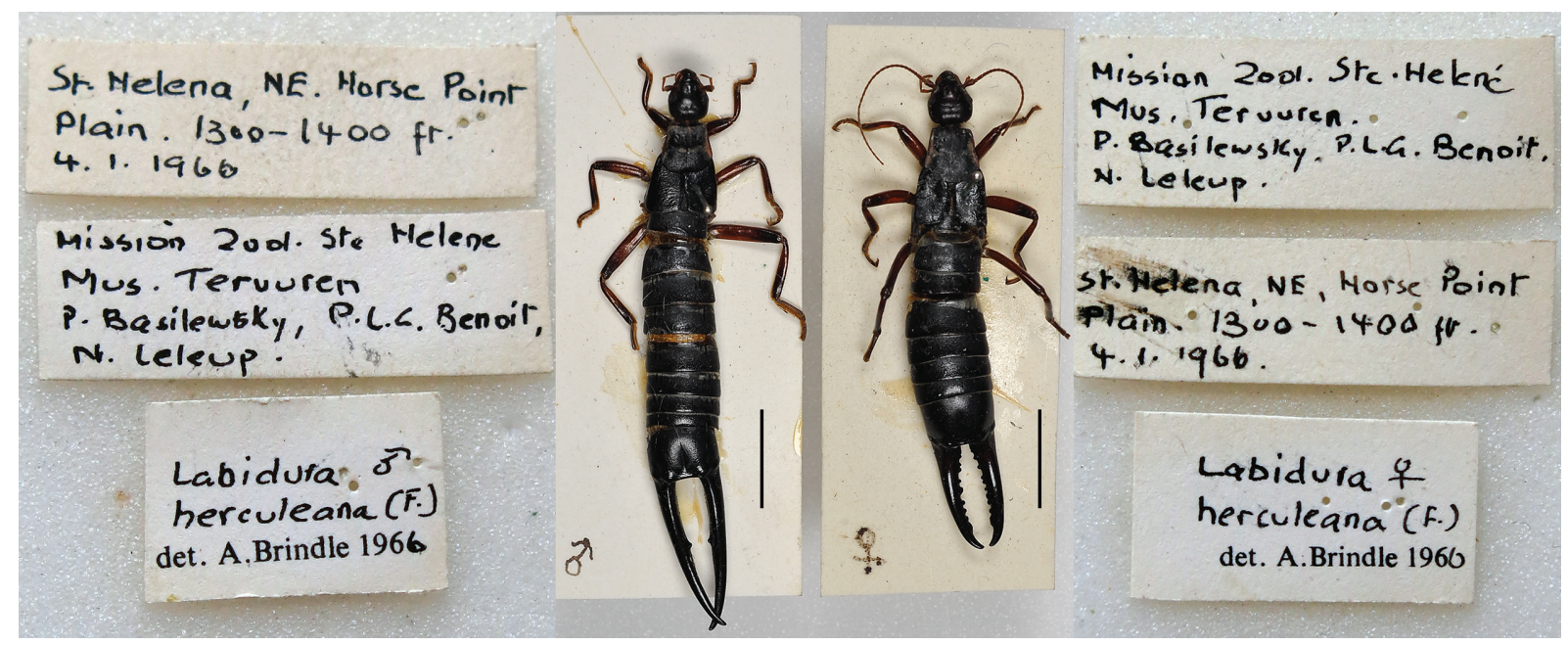

Fig. 12. Male and female specimens of Labidura herculeana (Fabricius, 1798), with corresponding data labels; MMUE, accession numbers F3233.1871 and F3233.1872. Scale bar $=1 \mathrm{~cm}$. 
them to L. loveridgei (Brindle 1970c), but was keen to see more material, according to his letter to Loveridge in June 1960 (MMUE, Hincks archive, item 360, Fig. 13). L. herculeana forceps were found with bird bones by Philip Ashmole and Douglas Dorward at Prosperous Bay in 1959. Other remains found by Philip and Myrtle Ashmole in 1995 suggest that some Giant Earwigs lived in seabird colonies in rocky places before the seabirds were eradicated by predators (Ashmole \& Ashmole 2004).

In 1960, Loveridge wrote to Hincks outlining his intention to search for further L. herculeana remains at the same spot where the forceps had been found, and wrote to him again six months later, recounting the unsuccessful results of his expedition (Fig. 14). Loveridge had worked at Manchester Museum in the time of J.R. Hardy. He said that if he had known Hincks was at MMUE, he would have asked for the forceps to be deposited there "...where I know the collections are well-cared for", rather than at the British Museum.

\section{Parasitic earwigs}

The collection also contains examples of parasitic earwig species. Two of the five species of the family Arixeniidae are present. There are 15 specimens of Arixenia esau Jordan, 1909 (11 in spirit, 4 dried and mounted) (Fig. 15) and 8 specimens of Xeniaria jacobsoni (Burr, 1912) (3 in spirit, 5 dried and mounted) (Fig. 16). Karl Jordan (1861-1959), who described the family Arixeniidae and also A. esau in 1909, sent 6 specimens of Arixenia (now Xeniaria) jacobsoni to Hincks, stating, "....the sender and collector are now in Paradise, where all good entomologists go" (MMUE, Brindle archive, item 55) (Fig. 17). Jordan's letter was sent from Walter Rothschild's natural history museum at the small market town of Tring in Hertfordshire, England. This museum, now part of the Natural History Museum, London, is still one of the largest private natural history collections ever assembled (Natural History Museum 2015c).

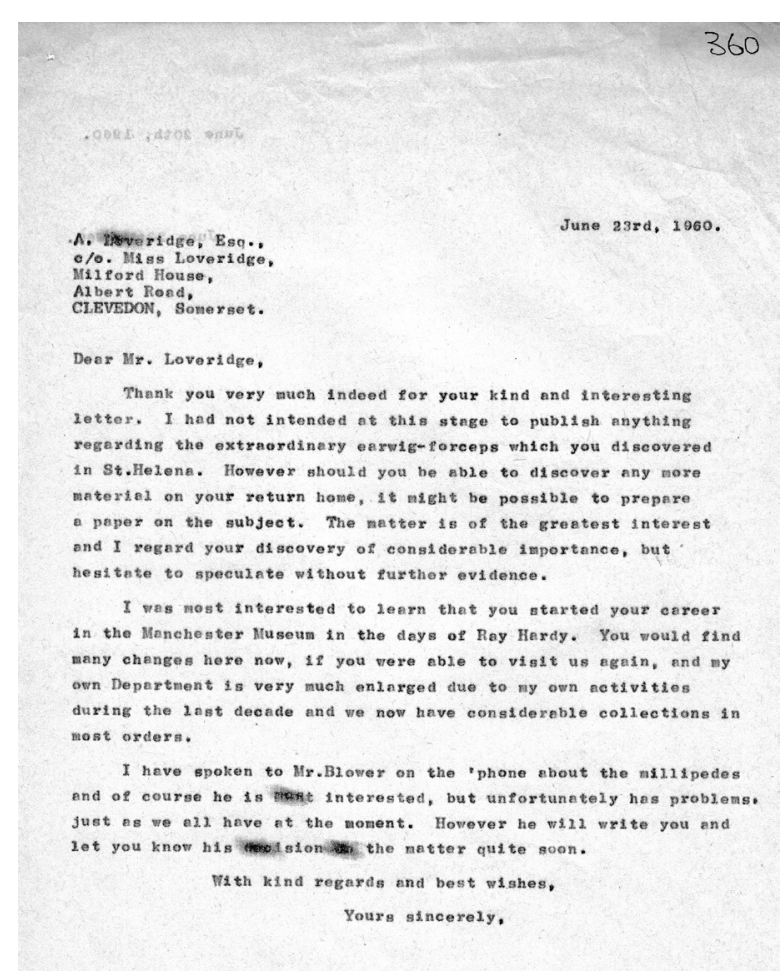

Fig. 13. Letter from Hincks to Arthur Loveridge, 23 Jun. 1960 (MMUE, Hincks archive, item $360)$.

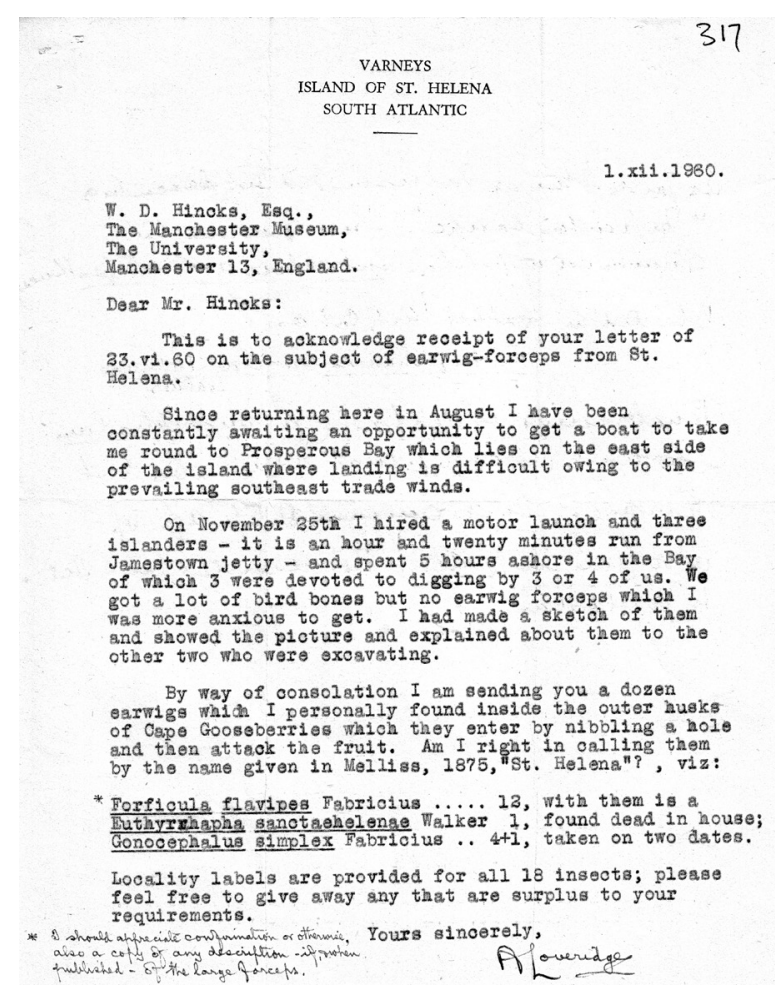

Fig. 14. Letter from Arthur Loveridge to Hincks relating his unsuccessful search for the Giant Earwig (MMUE, Hincks archive, item 317). 

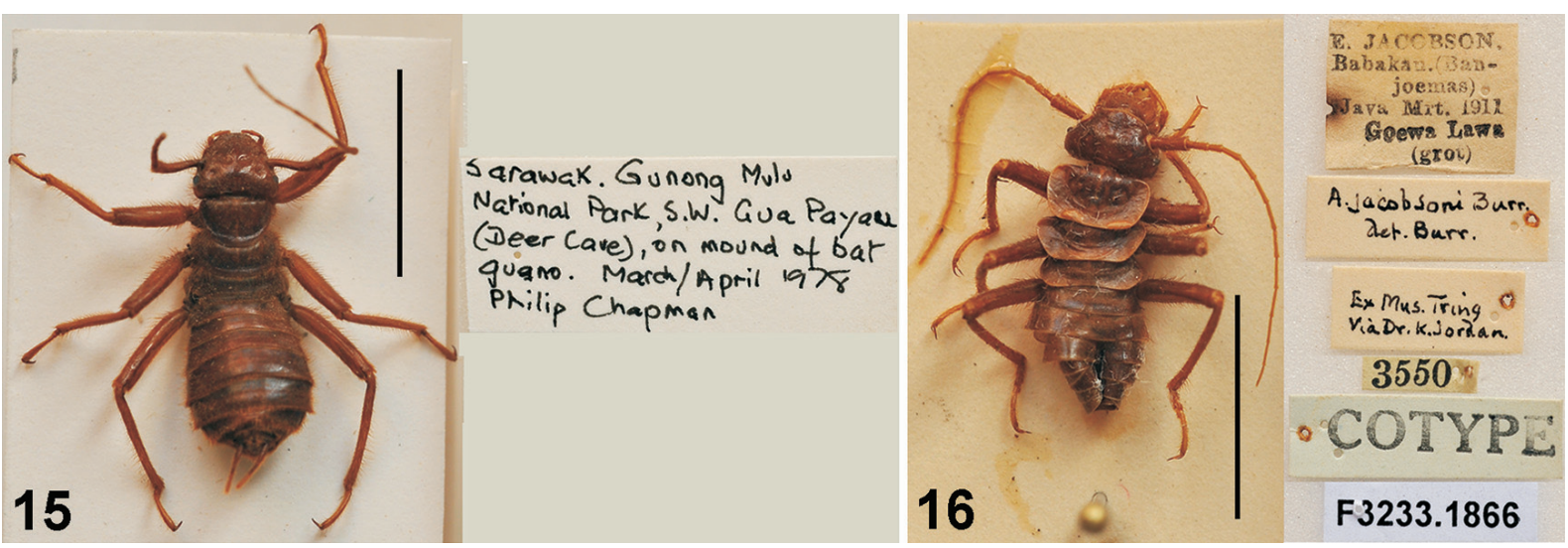

Figs 15-16. Parasitic earwigs from the collection of the Manchester Museum. 15. Arixenia esau Jordan, 1909. 16. A. jacobsoni Burr, 1912 (now Xeniaria jacobsoni), syntype. Scale bars $=1 \mathrm{~cm}$.

Jordan had been curator of insects there since 1893. At the date of the letter in 1947, Jordan was 86 years old, having continued as an 'unofficial worker' at Tring since 1939 (Johnson K.R. 2003: 314). All 5 specimens of $X$. jacobsoni now at MMUE are labelled 'Ex. Mus. Tring via Dr. K. Jordan'. Three are labelled 'Cotype' with Burr's determination labels and dated 1911. Another, collected in 1919 by a Dr Hacker, was found 'on bat' according to the specimen label.

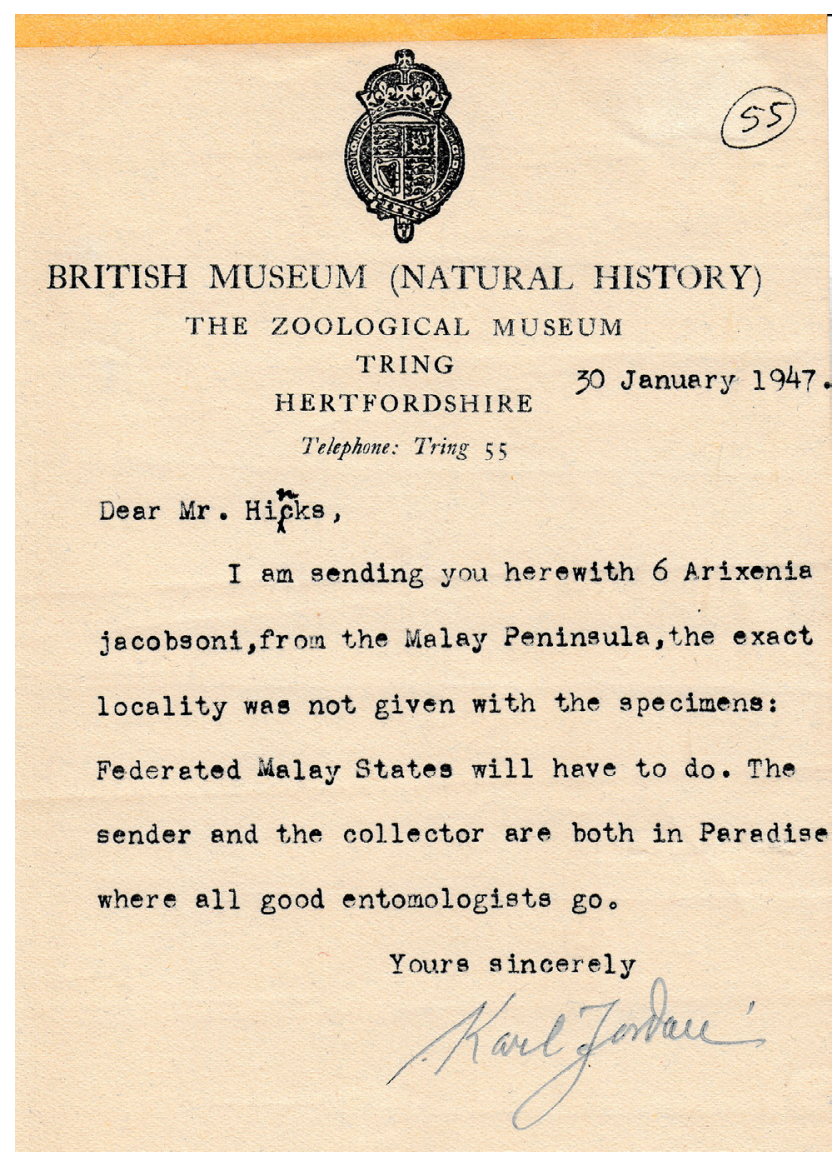

Fig. 17. Letter from Karl Jordan to Hincks enclosed with 6 'Arixenia jacobsoni' specimens (MMUE, Brindle archive, item 55). 
The Arixenia esau specimens come from Gua Payau (Deer Cave), Sarawak, the specimen label stating that they were found on a mound of bat guano. The wingless Arixeniidae occur in Indonesia, the Philippines and the Malay peninsula, and are associated with bats of the genus Cheiromeles, feeding on the bats' skin and gland secretions (Kocarek et al. 2013). They occur in large numbers on guano in caves (Brindle \& Oromi 1994). A. esau is not often found actually on flying bats, although it has been suggested this is how the earwigs transfer to new roosts (Marshall 1977).

The collection also contains 5 of the 11 species of the Hemimeridae (36 specimens, 15 in spirit), which are ectoparasites of Giant Rats. Hemimerus talpoides Walker, 1871 (Fig. 18) were the earliest recorded foreign Dermaptera in the Accession Register, in 1919. The family shows adaptations to a parasitic life with specialized grooves on the legs for close attachment to the host, loss of wings and eyes, and straight, narrow cerci (Kocarek et al. 2013). In a study by Mogbo \& Akunne (2014) in Awka, Nigeria, Hemimerus talpoides was found to be the most abundant parasite of Cricetomys gambianus rats, with up to 9 on those examined. These rats are economically important in the area as a source of protein and are sold for hide. Their keen sense of smell has been used to detect land mines in Tanzania and to diagnose tuberculosis (Mogbo \& Akunne 2014), so just how injurious the parasites are to their hosts may well be a topic for further research.

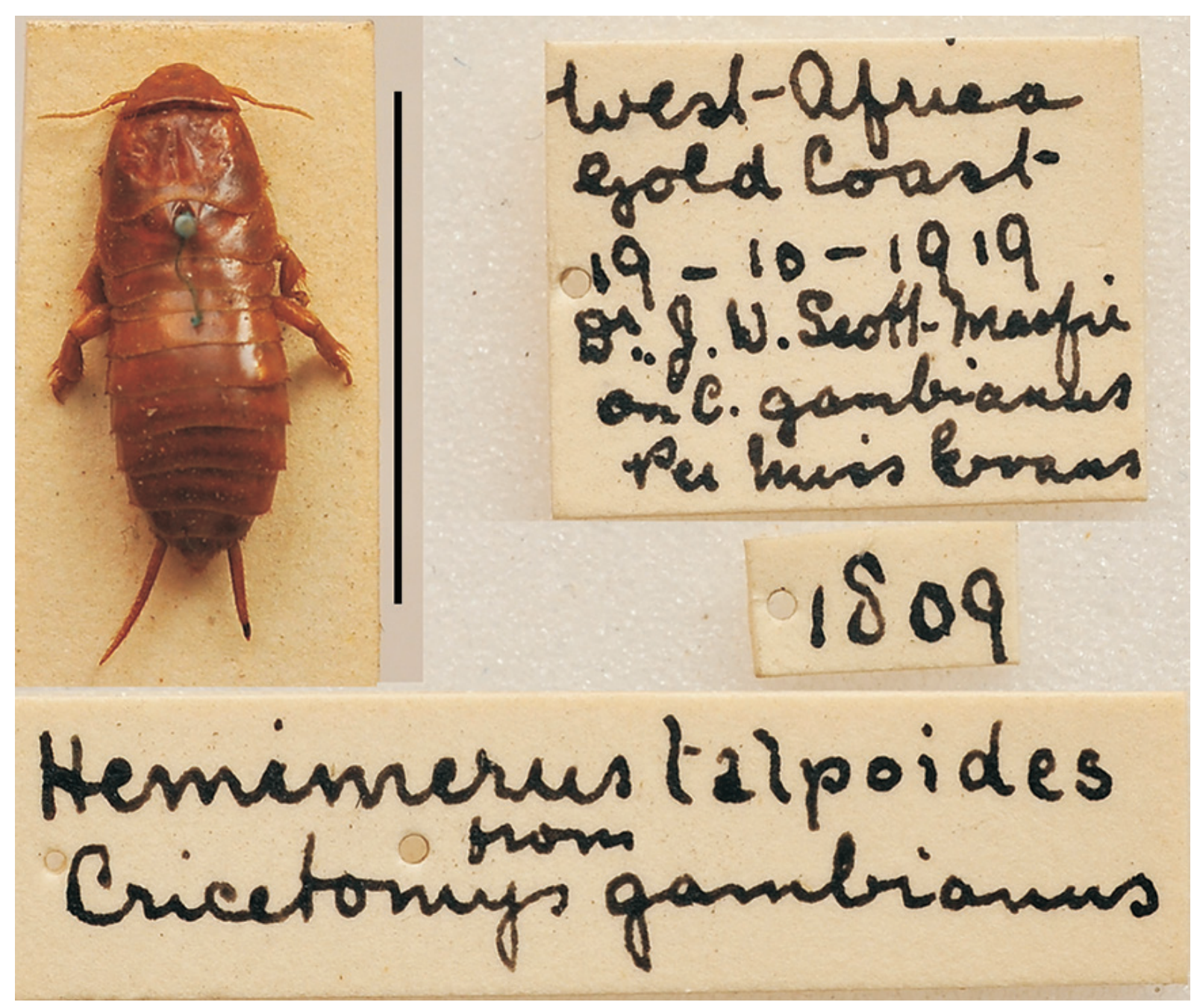

Fig. 18. The parasitic earwig Hemimerus talpoides Walker, 1871, the Manchester Museum. Scale bar = $1 \mathrm{~cm}$. 
Table 4. Number of Dermaptera species and subspecies represented by type specimens at the Manchester Museum, by family (nomenclature follows Steinmann 1989c, complemented by latest updates from Engel \& Haas 2007).

\begin{tabular}{lccccccc}
\hline \multirow{2}{*}{ Family } & \multicolumn{7}{c}{ Number of species and subspecies } \\
\cline { 2 - 6 } & $\begin{array}{c}\text { Holotype } \\
\text { only }\end{array}$ & $\begin{array}{c}\text { Holotype }+ \\
\text { paratype(s) }\end{array}$ & $\begin{array}{c}\text { Paratype(s) } \\
\text { only }\end{array}$ & $\begin{array}{c}\text { Syntype(s) } \\
\text { only }\end{array}$ & Total & $\begin{array}{c}\text { \% World } \\
\text { Fauna }\end{array}$ & $\begin{array}{c}\text { No. of } \\
\text { specimens }\end{array}$ \\
\hline Anisolabididae & 1 & 7 & 60 & 2 & 70 & 17.8 & 157 \\
Arixeniidae & 0 & 0 & 0 & 1 & 1 & 20.0 & 3 \\
Chelisochidae & 0 & 0 & 4 & 1 & 5 & 5.3 & 11 \\
Diplatyidae & 1 & 0 & 17 & 0 & 18 & 12.5 & 25 \\
Forficulidae & 1 & 5 & 43 & 1 & 50 & 10.6 & 129 \\
Karschiellidae & 0 & 0 & 2 & 0 & 2 & 16.7 & 6 \\
Labiduridae & 1 & 0 & 5 & 0 & 6 & 7.9 & 10 \\
Pygidicranidae & 2 & 3 & 25 & 1 & 31 & 16.8 & 81 \\
Spongiphoridae & 6 & 13 & 79 & 2 & 100 & 19.6 & 312 \\
Total & $\mathbf{1 2}$ & $\mathbf{2 8}$ & $\mathbf{2 3 5}$ & $\mathbf{8}$ & $\mathbf{2 8 3}$ & $\mathbf{1 5 . 0}$ & $\mathbf{7 3 4}$ \\
\hline
\end{tabular}

\section{Type catalogue of the Dermaptera collection at MMUE}

At the Manchester Museum, type material is kept within the main collection. In total there are 733 holotypes, allotypes, syntypes and paratypes, representing 283 species (Table 4). This includes 40 holotypes, of which 32 species were described by Brindle and 8 by Hincks.

The following catalogue provides a complete list of the holotypes, syntypes, allotypes and paratypes in the collection, arranged alphabetically by species name as originally described, in the following format:

\section{Species name, Author (Original genus)}

Literature citation for the original description.

\section{Type material}

Number, gender and type of specimens, accession number/s, mount.

Type locality

Transcription of holotype locality label.

\section{Locality label/s}

Transcription of locality label/s of other type specimens.

Taxonomy

Current nomenclature following Steinmann (1989c), unless otherwise stated, complemented with latest changes by Engel \& Haas (2007) and reference to the checklist of Hopkins et al. (2014).

\section{Remarks}

Including nomenclatural changes and any supplementary information from original descriptions and Brindle's Type Index Card system.

Also included in a separate section below is material labelled as type material, which is assumed to represent unpublished 'manuscript names' only.

The following notation is used: (/) - new line; (//) - separate specimen label; [ ] - editorial text. 
Abbreviations:

$\mathrm{BMNH}=$ British Museum (Natural History), UK

MMUE = The Manchester Museum, UK

STRI $=$ Smithsonian Tropical Research Institute

acutangula Hincks, 1955 (Dacnodes)

Dacnodes acutangula Hincks, 1955c: 807, figs 1-2.

\section{Type material}

1 , paratype, F3233.1318, dried, mounted on pin.

\section{Locality label}

Musée du Congo / Elizabethville / XII - 1932 / Mlle Kerkvoorde.

\section{Taxonomy}

Pygidicranidae, Pygidicraninae, Dacnodes acutangula Hincks, 1955.

\section{adjacens Hincks, 1955 (Diplatys)}

Diplatys adjacens Hincks, 1955a: 110, fig. 143.

\section{Type material}

1 Oे, paratype, F3233.1291, dried, mounted on card.

\section{Locality label}

W. Almora Divn / Kumaon U.P. / July 1917, HGC.

\section{Taxonomy}

Diplatyidae, Diplatyinae, Diplatys (Syndiplatys) adjacens Hincks, 1955.

\section{Remarks}

1. Transferred by Steinmann (1986a: 175).

2. The original description makes no reference to any paratype at MMUE.

3. The specimen carries Hincks' determination label, stating 'Paratype'.

4. The collector H.G. Champion is named in the description.

5. The locality Kumaun in the description, and Kumaon on the label, are variant spellings for a mountainous region in Uttar Pradesh, India.

6. Index card entry: UP = United Provinces, now Uttar Pradesh, India.

\section{affinis Hincks, 1947 (Diplatys)}

Diplatys affinis Hincks, 1947b: 12, fig. 6.

\section{Type material}

1 , paratype, F3233.1292, dried, mounted on card. 


\section{Locality label}

Burma ca 200 m. / Washaung, 20 km / East of Myitkyina / 14.VII.34, Malaise.

\section{Taxonomy}

Diplatyidae, Diplatyinae, Diplatys (Hypodiplatys) bormansi Burr, 1910.

\section{Remarks}

Diplatys affinis synonymised with Diplatys bormansi Burr, 1910 by Hincks (1955a: 124). Transferred by Steinmann (1986a: 175).

\section{africana Brindle, 1968 (Labia)}

(Fig. 50)

Labia africana Brindle, 1968e: 24, figs 13-15.

\section{Type material}

$1 \lesssim$, holotype, F3233.1590 (Fig. 50), dried, mounted on card.

\section{Type locality}

Nigeria: / Banchi Prov. / Gadan 25.6.57 / P. Blasdale.

\section{Taxonomy}

Spongiphoridae, Geracinae, Pseudovostox africanus (Brindle, 1968).

\section{Remarks}

Transferred by Brindle (1970g: 60). Hopkins et al. (2014) refer to this species as 'africana'. Steinmann (1989c: 424) states Brindle's use of $P$. africana in 1970g is a misprint for P. africanus. (See also below under P. africanus meridionalis and P. africanus sudanensis.)

\section{africanus Brindle, 1973 (Cordax)}

Cordax africanus Brindle, 1973f: 306, figs 417-420.

\section{Type material}

1 ऽ, paratype, F3233.1681, dried, mounted on card.

\section{Locality label}

S. Africa. / R. E. Turner. / Brit. Mus. / 1924-54. // Port St. John, / Pondoland. / Dec. 1923.

\section{Taxonomy}

Forficulidae, Opisthocosmiinae, Syntonus africanus (Brindle, 1973).

\section{Remark}

Transferred by Steinmann (1993: 250).

\section{africanus Brindle, 1978 (Osteulcus)}

Osteulcus africanus, Brindle, 1978: 194, fig. 349. 
Type material

1 , paratype, F3233.1745, dried, mounted on card.

\section{Locality label}

Cameroun / Yaoundé / Vadon! / IX.30.

\section{Taxonomy}

Forficulidae, Ancistrogastrinae, Osteulcus africanus Brindle, 1978.

\section{africanus meridionalis Brindle, 1970 (Pseudovostox)}

Pseudovostox africanus meridionalis Brindle, 1970e: 265.

\section{Type material}

1 , paratype, F3233.1591, dried, mounted on card.

\section{Locality label}

Coll. Mus. Congo / Tanganika: Musosa, 980m. / (à la lumiere) XI-1953 / H. Bomans.

\section{Taxonomy}

Spongiphoridae, Geracinae, Pseudovostox africanus meridionalis Brindle, 1970.

\section{Remarks}

1. Hopkins et al. (2014) refer to this species as 'africana'. Brindle named the subspecies P. africanus meridionalis in the original description and subsequently (Brindle 1973f: 136). Steinmann (1989c: 424) has $P$. africanus meridionalis.

2. Additional information on the index card: Tanzania, Musosa.

\section{africanus sudanensis Brindle, 1970 (Pseudovostox)}

Fig. 53

Pseudovostox africanus sudanensis Brindle, 1970e: 266.

\section{Type material}

1 ふै, holotype, F3233.1592 (Fig. 53), dried, mounted on card.

\section{Type locality}

Sudan: Yirol / 21.V.54 / E. T. M. Reid.

\section{Taxonomy}

Spongiphoridae, Geracinae, Pseudovostox africanus sudanensis Brindle, 1970.

\section{Remarks}

Hopkins et al. (2014) refer to this species as 'africana'. Brindle named the subspecies $P$. africanus sudanensis in the original description and subsequently (1973f: 138). Steinmann (1989c: 424) has $P$. africanus sudanensis. 


\section{albocincta Brindle, 1966 (Parablandex)}

Parablandex albocincta Brindle, 1966g: 232, figs 21-24.

\section{Type material}

1 ô, paratype, F3233.1339, dried, mounted on card.

\section{Locality label}

Madagascar / e Mus / Geneva.

\section{Taxonomy}

Pygidicranidae, Blandicinae, Parablandex albocincta Brindle, 1966.

\section{Remark}

The original description makes no reference to specimens at MMUE.

\section{apicalis Hincks, 1951 (Nesogaster)}

Nesogaster apicalis Hincks, 1951b: 568, figs 10-11.

\section{Type material}

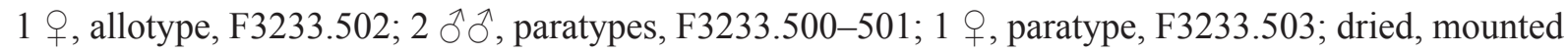
on card.

\section{Locality labels}

F3233.500: New Hebrides: / Malekula, / Ounua. / Feb. 1929 / Miss L. E. Cheesman. / B. M. 1929-280. F3233.501: New Hebrides: / Santo. / viii-ix. 1929 / L. E. Cheesman. / B. M. 1929-537.

F3233.502: New Hebrides: / N. E. Malekula / vii-1929 / L. E. Cheesman. / B. M. 1929-514.

F3233.503: New Hebrides: / Malekula, / Ounua. / Mar. \& Apl. 1929. / Miss L. E. Cheesman. / B. M. 1929-343.

\section{Taxonomy}

Spongiphoridae, Nesogastrinae, Nesogaster aculeatus (de Bormans, 1900).

\section{Remarks}

1. Nesogaster apicalis synonymised with N. aculeatus (de Bormans, 1900) by Steinmann (1989b: 23).

2. Locality Ounua given as 'Ounna' in the original description (possible mistranscription).

\section{aptera Brindle, 1978 (Geracodes)}

Geracodes aptera Brindle, 1978: 67, figs 81-83.

\section{Type material}

1 , , paratype, F3233.1444, dried, mounted on card.

\section{Locality label}

IFAN / Nimba (Guinée) / Lamotte et Roy / VII - XII 51. 


\section{Taxonomy}

Anisolabididae, Isolabidinae, Geracodes aptera Brindle, 1978.

\section{araguensis Brindle, 1974 (Geracides)}

Geracides araguensis Brindle, 1974b: 120, figs 11-12, 14, 21.

\section{Type material}

1 Oे, paratype, F3233.1531, dried, mounted on card.

\section{Locality label}

Venezuela - Aragua / Rancho Grande / 1100m. 14.VIII.73. // Col. J. L. Garcia.

\section{Taxonomy}

Spongiphoridae, Cosmogeracinae, Cosmogerax araguensis (Brindle, 1974).

\section{Remarks}

Taxonomy follows Steinmann 1989c. Transferred by Brindle (1982a: 36) as Cosmogerax araguensis. Steinmann (1989b: 100) has C. araguense. Hopkins et al. (2014) has C. araguensis.

\section{arcuatus Brindle, 1968 (Auchenomus)}

Auchenomus arcuatus Brindle, 1968h: 543, figs 5-6, 9.

\section{Type material}

1 Oे, paratype, F3233.1499, dried, mounted on card, without head.

\section{Locality label}

Ripang / N. Luzon.

\section{Taxonomy}

Spongiphoridae, Sparattinae, Auchenomini, Auchenomus arcuatus Brindle, 1968.

\section{Remark}

Index card entry: Philippine Islands.

\section{argentinus Hincks, 1959 (Esphalmenus)}

Fig. 36

Esphalmenus argentinus Hincks, 1959: 203, figs 202, 209.

\section{Type material}

$1 \hat{\jmath}$, holotype, F3233.1341 (Fig. 36); 1 क , allotype, F3233.1342; 4 $\widehat{\partial}$, paratypes, F3233.1343, F3233.1346-1348; 8 우, paratypes, F3233.1344-1345, F3233.1349 (2 specimens), F3233.1350 (2 specimens), F3233.1351 ( 2 specimens); 2 paratypes, gender not specified, F3233.1352; all dried, mounted on card.

\section{Type locality}

Argentine: / Laguna Cazmiril / Tucuman 4500m. / 10. XII.1947. / Coll. Sacriste. 


\section{Locality labels}

F3233.1342-1347, F3233.1349-1352: Argentina / Tucuman: Laguna / Cazmiril 4,500m. / 10. XII.1947 (Sacriste).

F3233.1348: Chile - Tarapaca / Arica / Tahapaca. 4800 / 22.2.48. // Coll. / Kuschel.

\section{Taxonomy}

Pygidicranidae, Esphalmeninae, Esphalmenus argentinus Hincks, 1959.

ashmolei Brindle, 1981 (Capralabis)

Capralabis ashmolei Brindle, 1981: 77, figs 1-2, 4, 7.

\section{Type material}

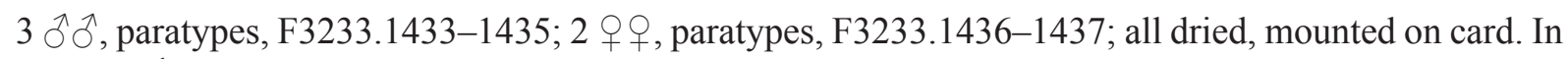

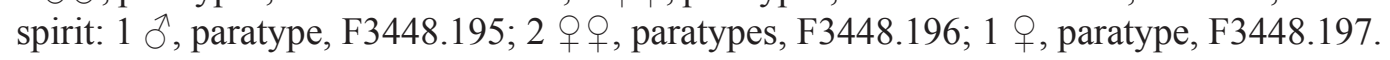

\section{Locality labels}

F3233.1433-1437: Ecuador / Los Tayos / Main cave / 12.7.1976 / N. P. Ashmole.

F3448.195: 25 Jul 76 / Los Tayos 815 78 12'W 306'S // Main cave. Bottom / of 2' [illegible]. Rotten wood / Threshold.

F3448.197: same data except number, 811.

F3448.196: 20 Jul 76 / $93078^{\circ} 12^{\prime} \mathrm{W} 3^{\circ} 06^{\prime} \mathrm{S} / /$ Bottom of 2' [illegible] shaft. / Under stones in / stream. Sta. 1.

\section{Taxonomy}

Anisolabididae, Anisolabidinae, Capralabis ashmolei Brindle, 1981.

\section{Remarks}

The original description refers to two male and four female paratypes at MMUE, rather than three males and two females; and in spirit, 1 , same data except 25 Jul. 1976, and 2 q $q$, in spirit, same data except 2 Jul. 1976. This last date may be a mistranscription of 20 for 2 . There is no reference to a male paratype in spirit.

\section{ater Brindle, 1988 (Pericomus)}

Fig. 52

Pericomus ater Brindle, 1988: 48, figs 1-2.

\section{Type material}

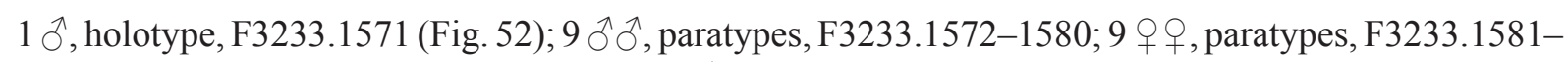

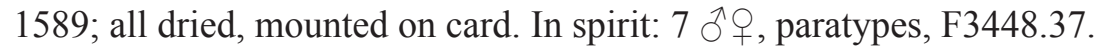

\section{Type locality}

Panama, Fortuna / Wet forest, / at light, 1050m / 28.6.1979 / Dr. Henk Wolda, STRI.

\section{Locality labels}

As for type, except for dates: F3233.1576, 28 Jul. 1977; F3233.1586, 1 Sep. 1977; F3233.1587, F3233.1589, 24 Sep. 1977; F3233.1580, 13 Oct. 1977; F3233.1582, 15 Nov. 1977; F3233.1584, 23 Jun. 1978; F3233.1583, 12 Jul. 1978; F3233.1573, 20 Jul. 1978; F3233.1574, 2 Aug. 1978; F3233.1578, 12 
Aug. 1978; F3233.1579, 4 Sep. 1978; F3233.1585, 5 Sep. 1978; F3233.1572, 20 Sep. 1978; F3233.1575, 6 May 1979; F3233.1577, F3233.1588, 15 Jun. 1979; F3233.1581, 25 Jun. 1979; F3448.37, 1977-1978.

\section{Taxonomy}

Spongiphoridae, Pericominae, Pericomus ater Brindle, 1988.

\section{Remarks}

1. The original description refers to 10 male and 10 female paratypes.

2. Dates of F3233.1576 (28 Jul. 1977) and F3233.1581 (25 Jun. 1979) do not match the date range in the description, 1 Aug. 1977-15 Jun. 1979.

\section{auchenomoides Hincks, 1954 (Chaetospania)}

Chaetospania auchenomoides Hincks, 1954e: 15, figs 5-7.

\section{Type material}

1 ڤึ, paratype, F3233.573; 1 \&, paratype, F3233.574; dried, mounted on card.

\section{Locality labels}

F3233.573-574: C. Sumba / Lokojengo / 22.9.49 / Bühler \& Sulter.

\section{Taxonomy}

Spongiphoridae, Sparattinae, Chaetospaniini, Chaetospania auchenomoides Hincks, 1954.

\section{Remark}

Index card entry: Lesser Sunda Islands.

\section{basidentatus Brindle, 1984 (Esphalmenus)}

Esphalmenus basidentatus Brindle, 1984: 287, figs 5, 13, 25.

\section{Type material}

1 ô, paratype, F3233.1353; 1 + , paratype, F3233.1354; dried, mounted on card.

\section{Locality labels}

F3233.1353-1354: Argentina, Chubut / SO. Esquel / La Hoya, 800-1350 m / 24.II.1979 Mision Cientifica Danesa.

\section{Taxonomy}

Pygidicranidae, Esphalmeninae, Esphalmenus basidentatus Brindle, 1984.

\section{basilewskyi Brindle, 1966 (Diplatys)}

Diplatys basilewskyi Brindle, 1966e: 30, figs 1, 4.

\section{Type material}

1 [đ̊?], paratype, F3233.1293, dried, mounted on card, without forceps. 


\section{Locality label}

Coll. Mus. Congo / Tanganyika Terr.: Bunduki / Uluguru Mts., moy. Mgeta / 1300 m. 30-IV/2-V-1957.

\section{Taxonomy}

Diplatyidae, Diplatyinae, Haplodiplatys basilewskyi (Brindle, 1966).

\section{Remarks}

1. Transferred by Steinmann (1986a: 171).

2. Gender is given as $\hat{\sigma}$ on the index card and in the original description, but unspecified on the specimen labels.

\section{beroni Brindle, 1982 (Diplatys)}

Diplatys beroni Brindle, 1982b: 165, figs 2-3.

\section{Type material}

1 ऽ, paratype, F3233.1313, dried, mounted on card.

\section{Locality label}

Nigeria / Jos Plateau / State. 5.X.1976 / P. Beron leg.

\section{Taxonomy}

Diplatyidae, Diplatyinae, Diplatys beroni Brindle, 1982.

\section{bhutanensis Brindle, 1975 (Diplatys)}

Diplatys bhutanensis Brindle, 1975c: 12, figs 7-8.

\section{Type material}

$1 \hat{\jmath}$, paratype, F3233.1294; 1 \&, paratype, F3233.1295; dried, mounted on card.

\section{Locality labels}

F3233.1294-1295: Chimakothi / 1900/2300 22/5 / Nat.-Hist. Museum / Basel - Bhutan / Expedition 1972.

\section{Taxonomy}

Diplatyidae, Diplatyinae, Haplodiplatys bhowmiki (Srivastava \& Saha, 1975).

\section{Remarks}

Diplatys bhutanensis synonymised with Diplatys bhowmiki (Srivastava \& Saha, 1975) by Srivastava (1982: 62). Transferred by Steinmann (1986a: 171).

\section{bhutanensis Brindle, 1975 (Forficula)}

Forficula bhutanensis Brindle, 1975c: 43, fig. 45.

\section{Type material}

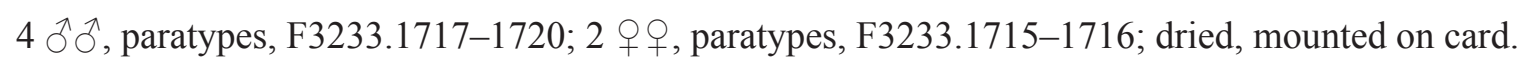




\section{Locality labels}

F3233.1715, F3233.1719: Gogona, 3100 m / 10-12.6 // Nat.-Hist Museum / Basel-Bhutan/Expedition 1972.

F3233.1716-1718, F3233.1720: Kotoka-Gogona / 2600-3400 m 10/6 // Nat.-Hist Museum / BaselBhutan / Expedition 1972.

\section{Taxonomy}

Forficulidae, Forficulinae, Forficula bhutanensis Brindle, 1975.

\section{Remark}

The original description states that three male paratypes from the first locality are at MMUE, not one male and one female; and two male and two female paratypes from the second locality, not one female and three males.

\section{bicarinata Hincks, 1947 (Guanchia)}

Guanchia bicarinata Hincks, 1947b: 28, fig. 14.

\section{Type material}

1 ô, paratype, F3233.1734; 1 \%, paratype, F3233.1735; dried, mounted on card.

\section{Locality labels}

F3233.1734: N. E. Burma / Kambaiti 7000 m / 12/5 1934 R. Malaise.

F3233.1735: As above except date 13 May 1934.

\section{Taxonomy}

Forficulidae, Forficulinae, Guanchia bicarinata Hincks, 1947.

\section{bicolor Brindle, 1973 (Archidux)}

Fig. 32

Archidux bicolor Brindle, 1973f: 296, fig. 402.

\section{Type material}

1 § , holotype, F3233.1697 (Fig. 32); 1 क, allotype, F3233.1698; 1 \&, paratype, F3233.1699; dried, mounted on card.

\section{Type locality}

Njombe / 6000-6500 ft. / Tanganyika / 20.2.1951 W. P.

\section{Locality labels}

As type except dates: F3233.1698, 22 Dec. 1951; F3233.1699, 18 Nov. 1951.

\section{Taxonomy}

Forficulidae, Skendylinae, Cosmiella bicolor (Brindle, 1973).

\section{Remarks}

1. Transferred by Steinmann (1989c: 669). 
2. The collection date for the allotype, F3233.1698, is given in the original description as 31.XII.1957. From inspection of the label it is probable that 1957 is a mistranscription of 1951.

3. The original description also refers to two paratypes, one male and one female, with the same data, dates 29 Oct. 1951 and 30 Nov. 1951, respectively.

\section{bicolor Brindle, 1975 (Brachylabis)}

Brachylabis bicolor Brindle, 1975b: 684, fig. 3.

\section{Type material}

1 , paratype, F3233.1466, dried, mounted on card.

\section{Locality label}

Tanzanie: Mts Uluguru / Chenzema, alt. 1700m. / 2-22/VII/71.

\section{Taxonomy}

Anisolabididae, Isolabidinae, Isolabis bicolor (Brindle, 1975).

\section{Remarks}

1. Transferred by Brindle (1978: 46).

2. The original description gives the date as Jun. 1971, not Jul. 1971 as on the specimen label.

\section{bidentata Brindle, 1971 (Strongylopsalis)}

Strongylopsalis bidentata Brindle, 1971b: 565, figs 90-92.

\section{Type material}

1 Oे, paratype, F3233.1616, forceps broken at tips; 1 q, paratype, F3233.1617; dried, mounted on card.

\section{Locality labels}

F3233.1616-1617: Colombia: D. E. Bogotá / El. 8,600 feet / 13-X-1964 / Susan Bates.

\section{Taxonomy}

Spongiphoridae, Strongylopsalinae, Strongylopsalis bidentata Brindle, 1971.

\section{Remark}

The original description gives the date as 23 Oct. 1964. Both labels are clearly amended to ' 13 ' over typed figures.

\section{bifurcatus Brindle, 1970 (Cordax)}

Cordax bifurcatus Brindle, 1970d: 23, figs 13-16.

\section{Type material}

1 , paratype, F3233.1682, dried, mounted on card.

\section{Locality label}

Belinga / Gabon. / 23.3.1963 / H. Coiffait. 


\section{Taxonomy}

Forficulidae, Opisthocosmiinae, Cordax bifurcatus Brindle, 1970.

\section{bilobatus Brindle, 1973 (Archidux)}

Archidux bilobatus Brindle, 1973f: 299, figs 407-410.

\section{Type material}

1 Oे, paratype, F3233.1700; 1 牛, paratype, F3233.1701; dried, mounted on pin.

\section{Locality labels}

F3233.1700-1701: 5 to 7 miles into / Kenia Forest. / Near Luchi R. / 9-10.2.1911.

\section{Taxonomy}

Forficulidae, Skendylinae, Cosmiella bilobata (Brindle, 1973).

\section{Remarks}

1. Transferred by Steinmann (1989c: 670).

2. Index card entry: Kenya.

\section{binodosa Brindle, 1967 (Gonolabina)}

Fig. 24

Gonolabina binodosa Brindle, 1967c: 9, figs 3, 5-6, 9, 10.

\section{Type material}

$1 \hat{\jmath}$, holotype, F3233.1451 (Fig. 24); 1 q, allotype, F3233.1452; 1 ㅇ, paratype, F3233.1453; dried, mounted on card.

\section{Type locality}

Zárate, Peru / Rio Rimac / 9.7.1953 / H. W. Koepcke.

\section{Locality labels}

As type locality.

\section{Taxonomy}

Anisolabididae, Gonolabininae, Gonolabina binodosa Brindle, 1967.

\section{bintumanensis Brindle, 1971 (Anisolabis)}

Anisolabis bintumanensis Brindle, 1971c: 272, figs 10-13.

\section{Type material}

1 Oे, paratype, F3233.1368; 1 \&, paratype, F3233.1369; dried, mounted on card.

\section{Locality labels}

F3233.1368: Savane 520 m / piste / [Keionaduga?] / 29.V.1963 Mission ENS-IFAN / aux Monts Loma / Sierra Leone. 
F3233.1369: Bintumane / $1870 \mathrm{~m}$ / 20.V.1963. // prairie à cyperacées / 20.V.1963. // Mission ENS-IFAN / aux Monts Loma / Sierra Leone.

\section{Taxonomy}

Anisolabididae, Anisolabidinae, Anisolabis bintumanensis Brindle, 1971.

\section{Remark}

The data label for F3233.1368 does not match any in the original description.

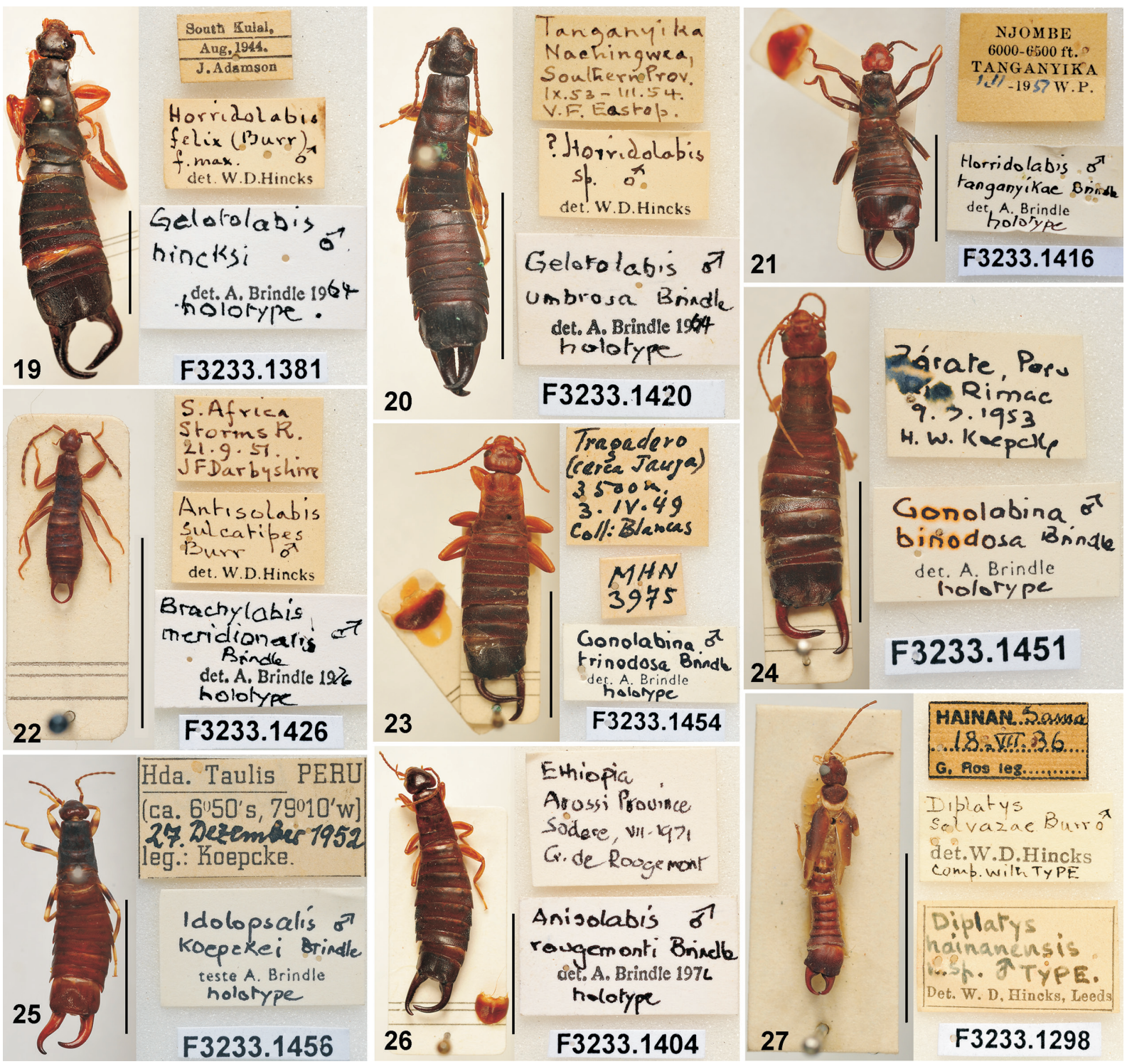

Figs 19-27. Holotypes $(\precsim)$ of Anisolabididae and Diplatyidae in the collection of the Manchester Museum. 19. Gelotolabis hincksi Brindle, 1964 (now Gonolabis hincksi). 20. G. umbrosa Brindle, 1964 (now Gonolabis umbrosa). 21. Gelotolabis tanganyikae Brindle, 1964 (now Gonolabis tanganyikae). 22. Brachylabis meridionalis Brindle, 1978 (now Antisolabis meridionalis). 23. Gonolabina trinodosa Brindle, 1967. 24. G. binodosa Brindle, 1967. 25. Idolopsalis koepckei Brindle, 1966. 26. Anisolabis rougemonti Brindle, 1978. 27. Diplatys hainanensis Hincks, 1940 (now Paradiplatys salvazae). Scale bars $=1 \mathrm{~cm}$. 


\section{bituberculata Brindle, 1966 (Anechura)}

Anechura bituberculata Brindle, 1966e: 43, figs 20-22.

\section{Type material}

$2 \widehat{\partial} \widehat{\jmath}$, paratypes, F3233.1721-1722; dried, mounted on card.

\section{Locality labels}

F3233.1721: Récolté dans / prairie sèche / avec buissons. // I.R.S.A.C.-Mus. Congo / Kivu: Terr. Mwenga, sud Lac / Lungwe, 2750 m. III-1953 / N. Leleup - G Marlier.

F3233.1722: I.R.S.A.C.-Mus. Congo / Kivu : Terr. Uvira, Ht Luvubu, 2750 m. (humus) / N. Leleup V-1954.

\section{Taxonomy}

Forficulidae, Forficulinae, Guanchia bituberculata (Brindle, 1966).

\section{Remarks}

1. Transferred by Steinmann (1993: 560).

2. In the original description, the locality of the specimen matching F3233.1721 is given as Lac Longwe, not Lac Lungwe and date as 1958, not 1953. For F3233.1722, locality given as Ht Lambo in original description, not Ht Luvubu as on specimen label.

\section{boliviana Brindle, 1971 (Strongylopsalis)}

Strongylopsalis boliviana Brindle, 1971f: 11, fig. 11.

\section{Type material}

1 ô, paratype, F3233.1618, dried, mounted on card.

\section{Locality label}

Quiaca // N. Holmgren.

\section{Taxonomy}

Spongiphoridae, Strongylopsalinae, Strongylopsalis excavata Brindle, 1973.

\section{Remarks}

1. S. excavata is a nomen novum provided by Brindle following the transfer of Carcinophora boliviana Bormans, 1903 to Strongylopsalis (Brindle 1973e: 14).

2. Index card entry: Bolivia.

\section{bolivianus Brindle, 1971 (Vostox)}

Fig. 40

Vostox bolivianus Brindle, 1971b: 541, fig. 46.

\section{Type material}

1 ऽ, holotype, F3233.1547 (Fig. 40), dried, mounted on card. 
Type locality

Bolivia 1100 m / Cochabamba / Alta Palmar / 3-1961 / leg. F. Walz.

\section{Taxonomy}

Spongiphoridae, Spongiphorinae, Vostox bolivianus Brindle, 1971.

\section{browni Hincks, 1954 (Labia)}

Labia browni Hincks, 1954c: 159, figs 1-2.

\section{Type material}

1 ऽ, paratype, F3233.1540, dried, mounted on card.

\section{Locality label}

Seychelles / Praslin: / Grande Anse / 26.V.52 / E.S. Brown.

\section{Taxonomy}

Spongiphoridae, Labiinae, Spirolabia browni (Hincks, 1954).

\section{Remark}

Transferred by Steinmann (1987: 180).

\section{brunneus Brindle, 1966 (Emboros)}

Emboros brunneus Brindle, 1966g: 255, figs 63, 67.

\section{Type material}

1 ऽ, paratype, F3233.1683; 1 + , paratype, F3233.1684; dried, mounted on card.

\section{Locality labels}

F3233.1683-1684: Périnet // Institut / Scientifique / Madagascar.

\section{Taxonomy}

Forficulidae, Skendylinae, Afrocosmia brunnea (Brindle, 1966).

\section{Remark}

Transferred by Steinmann (1975: 217).

\section{burri Hincks, 1955 (Cranopygia)}

Cranopygia burri Hincks, 1955c: 812, fig. 4.

\section{Type material}

1 ऽ, paratype, F3233.1316, dried, mounted on card.

\section{Locality label}

S. India / Madura Dis. / Shembaganur. / Astruc. 


\section{Taxonomy}

Pygidicranidae, Pygidicraninae, Cranopygia burri Hincks, 1955.

\section{burri Hincks, 1959 (Echinosoma)}

Echinosoma burri, Hincks, 1959: 158, figs 151-153.

\section{Type material}

1 ठิ, paratype, F3233.1320; 2 우, paratypes, F3233.1321-1322; dried, mounted on card.

\section{Locality labels}

F3233.1320-1321: Sumatra / Montagnes prés de / Pajakombo, mai 1905 // Coll. Gadeau de Kerville. F3233.1322: H. Lucht, Java / K. O Blawan / 2.3.39 / 900/1500 Mr. / Idjen Plateau.

\section{Taxonomy}

Pygidicranidae, Echinosomatinae, Echinosoma burri Hincks, 1959.

\section{Remark}

The original description refers to only one specimen at MMUE, corresponding to F3233.1322.

\section{burri Borelli, 1909 (Pseudisolabis)}

Pseudisolabis burri, Borelli, 1909: 1.

\section{Type material}

$1 \hat{\partial}$, syntype, F3233.1483, dried, mounted on card.

\section{Locality label}

Kaschmir.

\section{Taxonomy}

Spongiphoridae, Isolaboidinae, Isolaboides burri (Borelli, 1909).

\section{Remarks}

1. Transferred by Hincks (1958b: 132).

2. Additional information in the original description: “... raccolti lungo la strada che conduce da Sirinagur a Gilghit, N. E. del Kashmir; altitudine 2500 metri circa, maggio 1908."

\section{camposi Borelli, 1907 (Gonolabis)}

Gonolabis camposi Borelli, 1907a: 1, fig.

\section{Type material}

$1 \widehat{\jmath}$, syntype, F3233.1355, dried, mounted on pin.

\section{Locality label}

Chiquiboquio / Ecuador / Ex Coll. Borelli. 


\section{Taxonomy}

Pygidicranidae, Esphalmeninae, Esphalmenus camposi (Borelli, 1907).

\section{Remarks}

Transferred by Burr (1909: 252).

canaca Brindle, 1976 (Anisolabis)

Anisolabis canaca Brindle, 1976b: 70, fig. 3.

\section{Type material}

1 §ै, paratype, F3233.214; 1 + , paratype, F3233.215; dried, mounted on card.

\section{Locality labels}

F3233.214-215: New Caledonia: / P. D. Montague.

\section{Taxonomy}

Anisolabididae, Anisolabidinae, Anisolabis canaca Brindle, 1976.

\section{carinata Hincks, 1959 (Cranopygia)}

Cranopygia carinata Hincks, 1959: 65, fig. 50.

\section{Type material}

1 Oे, paratype, F3233.39; 1 \%, paratype, F3233.40; dried, mounted on pin.

\section{Locality labels}

F3233.39-40: Maffin Bay / Dutch New Guinea / IV-IX.44 / E. S. Ross Coll.

\section{Taxonomy}

Pygidicranidae, Pygidicraninae, Cranopygia carinata Hincks, 1959.

\section{carinata Brindle, 1978 (Isolabis)}

Isolabis carinata Brindle, 1978: 40, figs 33-34.

\section{Type material}

1 ô, paratype, F3233.1467; 1 q, paratype, F3233.1468; dried, mounted on card.

\section{Locality labels}

F3233.1467-1468: Récolté dans l'humus // I.R.S.A.C. / S. Rhodesia: Mt Selinda, / forêt de Chirinda, 1300 m. / VII.1960 N. Leleup.

\section{Taxonomy}

Anisolabididae, Brachylabidinae, Brachylabis carinata (Brindle, 1978).

\section{Remark}

Transferred by Steinmann (1989a: 303). 
carinatus Brindle, 1975 (Spongovostox)

Spongovostox carinatus Brindle, 1975b: 691, figs 10-11.

\section{Type material}

1 , paratype, F3233.1602, dried, mounted on card.

\section{Locality label}

Tanzanie: Mts Uluguru / Morogoro Campus Fac / Agric., piége lum. U.V. / alt. 600 m. V-VI/71. // Coll. Mus. Tervuren / Mission Mts Uluguru / L. Berger, N. Leleup / J. Debecker V/VIII/71.

\section{Taxonomy}

Spongiphoridae, Spongiphorinae, Spongovostox carinatus Brindle, 1975.

carinatus Brindle, 1977 (Vostox)

Vostox carinatus Brindle, 1977b: 122, figs 27-28.

\section{Type material}

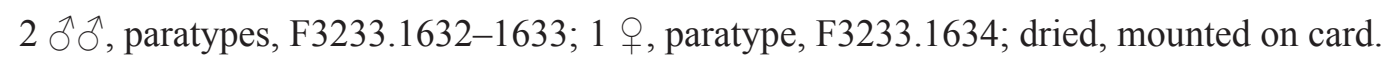

\section{Locality labels}

F3233.1632-1633: Venezuela, Aragua / Rancho Grande / La Trilla. Periquito / 11-I-1975. F3233.1634: Venezuela, Aragua / Rancho Grande / 1100m. 11-I-74.

\section{Taxonomy}

Spongiphoridae, Spongiphorinae, Vostox carinatus Brindle, 1977.

\section{Remark}

The original description states that one male and one female paratype at MMUE are from the La Trilla locality, not two males as in the collection.

\section{cavernicola Brindle, 1979 (Mixocosmia)}

Mixocosmia cavernicola Brindle, 1979: 153, fig. 6.

\section{Type material}

1 Oे, paratype, F3233.1778; 1 q, paratype, F3233.1779; dried, mounted on card.

\section{Locality labels}

F3233.1778-1779: Cuevas de la Puente / 3000 m. 20 km. S. / San Francisco, 40 km / E. San Luis Potosi / S. L. P. Mexico. 16-V-1972. // W. M. Elliott / R. Ralph / M. McEachern.

\section{Taxonomy}

Forficulidae, Skendylinae, Mixocosmia cavernicola Brindle, 1979.

\section{celisi Brindle, 1966 (Vandex)}

Vandex celisi Brindle, 1966c: 46, figs 3-4, 10. 


\section{Type material}

1 ऽ, paratype, F3233.1622; 1 + , paratype, F3233.1623; dried, mounted on card.

\section{Locality labels}

F3233.1622-1623: Dans bois mort. // Coll. Mus. Congo / Uganda: E. Ruwenzori, / Kalengire, 2000 m. / R. P. M. J. Celis 14-I-54.

\section{Taxonomy}

Spongiphoridae, Vandicinae, Vandex celisi Brindle, 1966.

\section{ceylonicus Brindle, 1977 (Nannisolabis)}

Nannisolabis ceylonicus Brindle, 1977a: 456, figs 1-3.

\section{Type material}

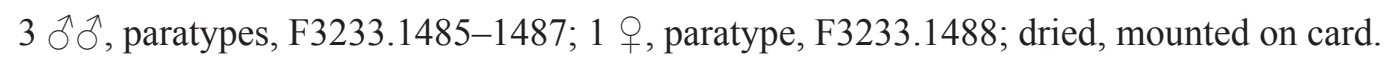

\section{Locality labels}

F3233.1485-1488: Ceylon, Central / Kandy: env. 600 m. / 19.I.1970 / C. Beaucheti I. Löbl.

\section{Taxonomy}

Anisolabididae, Antisolabidinae, Antisolabis ceylonica (Brindle, 1977).

\section{Remarks}

Transferred by Steinmann (1989a: 368). Steinmann (1989a) uses both 'Antisolabis ceylonicus' (p. 342) and 'Antisolabis ceylonica' (p. 368). Steinmann (1989a: 368, 1989c: 331) gives Brindle's original name for the species as Nannisolabis ceylonica, not Nannisolabis ceylonicus as in Brindle's original description (1977a: 456).

\section{chopardi Hincks, 1947 (Forficula)}

Forficula chopardi Hincks, 1947a: 201, fig. 1.

\section{Type material}

1 , paratype, F3233.1723, dried, mounted on card.

\section{Locality label}

Museum Paris / Mt. / Bintumani / 1800 m. // Museum Paris / Sierra Leone / P. Jaeger.

\section{Taxonomy}

Forficulidae, Forficulinae, Forficula chopardi Hincks, 1947.

\section{Remarks}

Steinmann (1993: 596), in the entry for F. chopardi, cites '1947 Anechura chopardi Hincks' as the original described taxon. This should read ' 1947 Forficula chopardi Hincks'. Hincks described Anechura chopardi in 1958 (Hincks 1958a: 185). Brindle (1973f: 270) transferred Anechura chopardi to Forficula with the new name $F$. borellii ( $F$. chopardi Hincks, 1947 being the senior homonym). In Steinmann 
1989c the two species, F. borellii Brindle, 1973 (p. 815) and F. chopardi Hincks, 1947 (p. 817) are clearly distinguished.

clavijoi Brindle, 1974 (Eugerax)

Eugerax clavijoi Brindle, 1974b: 119, fig. 20.

\section{Type material}

1 , paratype F3233.1532, dried, mounted on card.

\section{Locality label}

Venezuela - Aragua / Rancho Grande / 1100m. 17.V.73. // J. Salcedo / J. Clavijo.

\section{Taxonomy}

Spongiphoridae, Geracinae, Eugerax clavijoi Brindle, 1974.

\section{coalescens Borelli, 1927 (Chelisoches)}

Chelisoches coalescens Borelli, 1927: 75, figs 6-9.

\section{Type material}

3 우, syntypes, F3233.1676, dried, mounted on card.

\section{Locality label}

Fort de Kock / (Sumatra) 920 M. / 1925 / leg. E. Jacobson. // $q$ // Turin Mus. / ex spt.

\section{Taxonomy}

Chelisochidae, Chelisochinae, Proreus coalescens (Borelli, 1927).

\section{Remark}

Transferred by Hebard (1929: 338).

\section{coiffaiti Brindle, 1970 (Brachylabis)}

Brachylabis coiffaiti Brindle, 1970d: 14, figs 1-2, 5.

\section{Type material}

$1 \widehat{\partial}$, paratype, F3233.1469, dried, mounted on card.

\section{Locality label}

Belinga / 46 / H. Coiffait // Mission Biologique / Au Gabon / P P Grasse Directeur.

\section{Taxonomy}

Anisolabididae, Isolabidinae, Isolabis coiffaiti (Brindle, 1970).

\section{Remark}

Transferred by Brindle (1978: 52). 


\section{collinsi Brindle, 1980 (Brachylabis)}

Brachylabis collinsi Brindle, 1980: 172, figs 7, 9.

\section{Type material}

1 ô, paratype, F3233.104, dried, mounted on card.

\section{Locality label}

Sarawak / Gunong Mulu / National Park / 1978 N. M. Collins / Loc. D.

\section{Taxonomy}

Anisolabididae, Brachylabidinae, Brachylabis collinsi Brindle, 1980.

\section{Remark}

Additional information in the original description: 'Dipterocarp Forest 220 m February-March 1978'.

compressa Borelli, 1907 (Anisolabis)

Anisolabis compressa Borelli, 1907b: 3.

\section{Type material}

1 ${ }^{\lambda}$, syntype, F3233.1440, dried, mounted on card.

\section{Locality label}

1906. Bimbia / Viag. Ruwenzori, S.A.R.D. Abruzzi.

\section{Taxonomy}

Anisolabididae, Anisolabidinae, Euborellia compressa (Borelli, 1907).

\section{Remarks}

1. Transferred by Burr (1915: 544).

2. Index card entry: Uganda.

\section{confusus Hincks, 1955 (Diplatys)}

Diplatys confusus Hincks, 1955a: 103, figs 129-130.

\section{Type material}

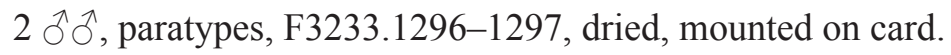

\section{Locality labels}

F3233.1296: S. India. / Madura Dis. / Shembaganur. / Astruc.

F3233.1297: S. India / Nilgiri Hills / Moyar Camp / 2,600' 10.4.54 / P.S. Nathan.

\section{Taxonomy}

Diplatyidae, Diplatyinae, Diplatys (Syndiplatys) confusus Hincks, 1955.

\section{Remark}

Transferred by Steinmann (1986a: 175). 


\section{convolutum Hincks, 1959 (Echinosoma)}

Echinosoma convolutum Hincks, 1959: 152, figs 140-141.

\section{Type material}

1 ô, paratype, F3233.1325, dried, mounted on card.

\section{Locality label}

Teinzo / Birmania / Fea, Maggio 1886.

\section{Taxonomy}

Pygidicranidae, Echinosomatinae, Echinosoma convolutum Hincks, 1959.

\section{cornutus Brindle, 1973 (Spongovostox)}

Spongovostox cornutus Brindle, 1973f: 207, figs 271, 277-278.

\section{Type material}

$2 \widehat{\jmath}$, paratypes, F3233.1603-1604; 1 + , paratype, F3233.1605; dried, mounted on card.

\section{Locality labels}

F3233.1603: Nigeria 10-IX 1966 / Univ. of Ife / Coll. J. L. Libby.

F3233.1604-1605: Dundo, Angola / 18-IX-1971 / A. De B. Machado. // From old termitory / of Macrotermes bellicosus.

\section{Taxonomy}

Spongiphoridae, Spongiphorinae, Spongovostox cornutus Brindle, 1973.

\section{cristata Hincks, 1954 (Apolabis)}

Apolabis cristata Hincks, 1954b: 104, figs 2-5.

\section{Type material}

$3 \hat{\partial} \widehat{\partial}$, paratypes, F3233.1370-1372; 2 우, paratypes, F3233.1373-1374; dried, mounted on card.

\section{Locality labels}

F3233.1370-1374: Mt To (1600 m) / Camp 1. / Museum Paris / Nimba (Guinée) / M. Lamotte II.VI.42.

\section{Taxonomy}

Anisolabididae, Anisolabidinae, Anisolabis cristata (Hincks, 1954).

\section{Remarks}

1. Transferred by Brindle (1978: 70).

2. In the original description, the date is given as Feb.-Apr. 1942, not Feb. Jun. 1942 as on the specimen labels.

\section{cristata Brindle, 1976 (Nesogaster)}

Nesogaster cristata Brindle, 1976b: 79, figs 24-25. 


\section{Type material}

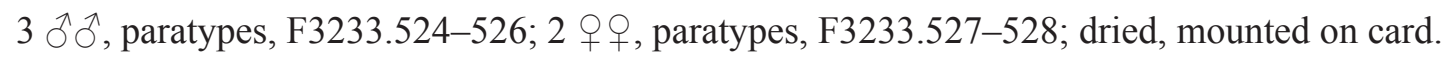

\section{Locality labels}

F3233.524: New Caledonia / Mt. Koghi / 27.I.1963. // C. Yoshimoto / \& N. Krauss / Light Trap. F3233.525: New Caledonia / Plateau de / Dogny. // 30. III.1968 // J. L. Gressitt / Collector / Bishop Museum.

F3233.526-527: New Caledonia / Plaine des Lacs / area, 6.XI.1958. // C.R. Joyce / Collector. F3233.528: New Caledonia / Foret de Thi to / Hamma, 700-800 m / 16.VII.1958. // Malkin \& J. Rageau / Collectors.

\section{Taxonomy}

Spongiphoridae, Nesogastrinae, Nesogaster cristatus Brindle, 1976.

\section{Remarks}

1. Both Steinmann (1989c: 404) and Hopkins et al. (2014) refer to this species as cristatus. Brindle gives cristata in the original description and on the 1973 determination labels.

2. In the original description, the data for F3233.524 only matches that for one of the specimens described under 'Other material examined' as being at MMUE, a larva.

\section{cunicula Brindle, 1978 (Anisolabis)}

Anisolabis cunicula Brindle, 1978: 113, figs 113-115, 239.

\section{Type material}

1 ऽึ, paratype, F3233.1375, dried, mounted on card.

\section{Locality label}

Nigeria Oluduce / S. E. State 1.III.1971 / Col. J. T. Medler.

\section{Taxonomy}

Anisolabididae, Anisolabidinae, Anisolabis cunicula Brindle, 1978.

\section{curta Brindle, 1975 (Tagalina)}

Fig. 38

Tagalina curta Brindle, 1975a: 151, fig. 5.

\section{Type material}

1 ô, holotype, F3233.60 (Fig. 38); 1 क, allotype, F3233.61; dried, mounted on pin.

\section{Type locality}

T.P.N.G. / 17.VI.56 / Tari / Dr. Peters.

\section{Locality label}

F3233.61: T.P.N.G. / 17.VI.56 / Tari / Dr. Peters.

\section{Taxonomy}

Pygidicranidae, Pygidicraninae, Tagalina curta Brindle, 1975. 


\section{Remarks}

1. Additional information in the original description: locality New Guinea.

2. The collection date is given in the description as 17 Jul. 1956, not 17 Jun. 1956.

\section{decellei Brindle, 1968 (Labia)}

Labia decellei Brindle, 1968d: 18, figs 8-10.

\section{Type material}

1 đ , paratype, F3233.1606; 1 ㅇ, paratype, F3233.1607; dried, mounted on card.

\section{Locality labels}

F3233.1606: Coll. Mus. Tervuren / Côte d'Ivoire : Bingerville / XI.1962 / J. Decelle.

F3233.1607: As above except date: Jun. 1961.

\section{Taxonomy}

Spongiphoridae, Spongiphorinae, Spongovostox decellei (Brindle, 1968).

\section{Remarks}

1. Transferred by Brindle (1973f: 195).

2. The original description does not refer to any female paratype examined other than the allotype, the collection date for which is Jul. 1961 (not Jun. 1961 as for $q$ paratype F3233.1607).

\section{delicatula Brindle, 1970 (Chaetospania)}

Chaetospania delicatula Brindle, 1970d: 19, figs 8-9.

\section{Type material}

$2 \hat{\jmath} \widehat{o}$, paratypes, F3233.1506-1507; 1 क , paratype, F3233.1508; dried, mounted on card.

\section{Locality labels}

F3233.1506: Belinga / 22.6.1963 / H. Coiffait // Mission Biologique / Au Gabon / PP Grasse Directeur. F3233.1507-1508: Same data except date, 22 Mar. 1963.

\section{Taxonomy}

Spongiphoridae, Labiinae, Chaetolabia delicatula (Brindle, 1970).

\section{Remark}

Transferred by Brindle (1973f: 164).

\section{delicatula Brindle, 1970 (Gressittolabis)}

Gressittolabis delicatula Brindle, 1970f: 687, figs 63-66.

\section{Type material}

$1 \widehat{\partial}$, paratype, F3233.929; 2 우, paratypes, F3233.930-931; dried, mounted on card. 


\section{Locality labels}

F3233.929-930: Solomon Is. / Guadacanal [sic] / Mt. Austen / Sept/Oct. 1965.

F3233.931: Bougainville, / Simba Mission / 2-VII-1956 / E. J. Ford.

\section{Taxonomy}

Chelisochidae, Chelisochinae, Gressittolabis delicatula Brindle, 1970.

\section{Remarks}

1. Steinmann (1989c) spells the genus 'Gressitolabis' and Hopkins et al. (2014) refer to Gressittolabis as a synonym of Gressitolabis. Brindle named the genus Gressittolabis for Dr J.L. Gressitt in the original description.

2. The original description gives the date for paratype F3233.931 as 2 Aug. 1965, not 2 Jul. 1956 as on the specimen label.

\section{dentatus Hincks, 1959 (Esphalmenus)}

Fig. 37

Esphalmenus dentatus Hincks, 1959: 205, fig. 210.

\section{Type material}

1 ô, holotype, F3233.1356 (Fig. 37); dried, mounted on card.

\section{Type locality}

Chile / Ex. S. / Kuschel.

\section{Taxonomy}

Pygidicranidae, Esphalmeninae, Esphalmenus dentatus Hincks, 1959.

\section{dentatus Brindle, 1988 (Vostox)}

Fig. 42

Vostox dentatus Brindle, 1988: 50, figs 7-8.

\section{Type material}

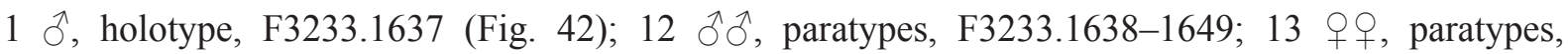
F3233.1650-1662; dried, mounted on card. In spirit: 18 $\widehat{\jmath}, 12$ 우, paratypes, F3448.46.

\section{Type locality}

Panama, Fortuna / Wet forest / at light, 1050m / 1.9.1976 / Dr. Henk Wolda, STRI.

\section{Locality labels}

As for type, except dates: F3233.1651, 28 Sep. 1976; F3233.1643, F3233.1648, 11 Oct. 1976; F3233.1646, F3233.1650, 17 Nov. 1976; F3233.1638, F3233.1652, F3233.1659, 21 Nov. 1976; F3233.1647, 23 May 1977; F3233.1661, 5 Aug. 1977; F3233.1660, 16 Aug. 1977; F3233.1644, 25 Spe. 1977; F3233.1658, 27 Sep. 1977; F3233.1642, 3 Jun. 1978; F3233.1655, 15 Jul. 1978; F3233.1649, 12 Sep. 1978; F3233.1662, 21 Sep. 1978; F3233.1640-1641, 25 Sep. 1978; F3233.1639, F3233.1656, 11 Dec. 1978; F3233.1657, 13 Nov. 1978; F3233.1653, 3 May 1979; F3233.1645, F3233.1654, 1 Jun. 1979. 
F3448.46: Panama : Chiriqui Province / Fortuna. 1050 m. at light / 1977-1978. Dr. Henk Wolda / Smithsonian Tropical Research Institute / Vostox dentatus Brindle / Paratypes 18 ô, 12 / det. A. Brindle 1980.

\section{Taxonomy}

Spongiphoridae, Spongiphorinae, Vostox dentatus Brindle, 1988.

\section{Remark}

The original description refers to 12 female paratypes at MMUE, not 13. Dates for all the MMUE specimens fall within the range given in the description (28.ix.1976-1.vi.1979) and the locality data match.

\section{denticulatum Hincks, 1959 (Echinosoma)}

Echinosoma denticulatum Hincks, 1959: 150, figs 136-137.

\section{Type material}

1 Oे, paratype, F3233.1323; 1 q, paratype, F3233.1324: dried, mounted on card.

\section{Locality labels}

F3233.1323-1324: Sumatra / Mont Ophir / Coll. Gadeau / de Kerville.

\section{Taxonomy}

Pygidicranidae, Echinosomatinae, Echinosoma denticulatum Hincks, 1959.

\section{Remark}

The original description has the spelling 'Mt. Opphir'. (Ophir (also known as Talakmau or Talamau) is a volcano in West Sumatra, Indonesia.)

\section{diagonalis Brindle, 1982 (Cosmogerax)}

Cosmogerax diagonalis Brindle, 1982a: 37, figs 3-6, 12.

\section{Type material}

2 q, , paratypes, F3233.1674-1675, dried, mounted on card.

\section{Locality labels}

F3233.1674-1675: Venezuela, Aragua / El Limon. 450 m. / 10-XII-1975.

\section{Taxonomy}

Spongiphoridae, Cosmogeracinae, Cosmogerax diagonalis Brindle, 1982.

\section{Remarks}

1. Brindle (1982a: 38) refers to only 1 , paratype at MMUE.

2. Taxonomy follows Steinmann 1989c. Steinmann (1989b: 100) has Cosmogerax diagonale. Hopkins et al. have C. diagonalis. 


\section{discalis Brindle, 1989 (Carcinophora)}

Carcinophora discalis Brindle, 1989: 14, fig. 17.

\section{Type material}

1 , paratype, F3233.1392, dried, mounted on card.

\section{Locality label}

Cerro Jele, Prov. Pana. / mà. enraiz de He- / lecho. Rep. Panama. / 12 Sept. 1985 / Col. Grupo Botanica.

\section{Taxonomy}

Anisolabididae, Anisolabidinae, Carcinophora discalis Brindle, 1989.

\section{Remark}

Not listed in Steinmann (1989c) or Hopkins et al. (2014).

\section{discedens Hincks, 1959 (Bormansia)}

Bormansia discedens Hincks, 1959: 170, fig. 166.

\section{Type material}

3 ठึ, paratypes, F3233.1334, F3233.1335 (2 specimens on one pin); 2 우, paratypes, F3233.1336; dried, mounted on card.

\section{Locality labels}

F3233.1334-1336: Mozambique / Luabo I.1957 / Miss P. J. Usher.

\section{Taxonomy}

Karschiellidae, Bormansia discedens Hincks, 1959.

\section{Remarks}

1. Listed in Steinmann (1989c: 115) and Hopkins et al. (2014) as discendens. Named 'discedens' in the original description and on Hincks' specimen determination labels.

2. The original description gives the locality as Luaba (possible misspelling of Luabo, Mozambique).

\section{distendens Brindle, 1975 (Forficula)}

Forficula distendens Brindle, 1975c: 47, figs 54-55.

\section{Type material}

1 , paratype, F3233.1733, dried, mounted on card.

\section{Locality label}

20 km S / Thimphu / 2,300 m 18/5 // Nat.-Hist Museum / Basel - Bhutan / Expedition 1972.

\section{Taxonomy}

Forficulidae, Forficulinae, Guanchia distendens (Brindle, 1975). 


\section{Remarks}

1. Transferred by Steinmann (1993: 585).

2. The original description gives the collection date as 31 May 1972, not 18 May 1972.

\section{distincta Brindle, 1975 (Chaetospania)}

Chaetospania distincta Brindle, 1975b: 688, fig. 8.

\section{Type material}

$1 \hat{\jmath}$, paratype, F3233.1509, dried, mounted on card.

\section{Locality label}

Tanzanie: Mts Uluguru / Kiroka, for. héliophile / alt. 725m 27-31/V/71.

\section{Taxonomy}

Spongiphoridae, Sparattinae, Chaetospaniini, Chaetospania distincta Brindle, 1975.

\section{diversus Hincks, 1953 (Emboros)}

Emboros diversus Hincks, 1953: 378, figs 21-22.

\section{Type material}

1 ô, paratype, F3233.1685; 1 + , paratype, F3233.1686; dried, mounted on card.

\section{Locality labels}

F3233.1685: Madagascar: / Mt. Tsaratanana / 1800 m. Forêt de / mousses. X.49 / R. Paulian. F3233.1686: Same data except altitude $1500 \mathrm{~m}$.

\section{Taxonomy}

Forficulidae, Skendylinae, Paracosmiella diversa (Hincks, 1953).

\section{Remark}

Transferred by Steinmann (1993: 167).

\section{doesburgi Brindle, 1982 (Cosmogerax)}

Cosmogerax doesburgi Brindle, 1982a: 38, fig. 2.

\section{Type material}

1 , paratype, F3233.1673; dried, mounted on card.

\section{Locality label}

Museum Leiden / Suriname Para Distr. / Onverdacht, on light / 7.VII.1962, 19-22h. / P.H.V. Doesburg jr.

\section{Taxonomy}

Spongiphoridae, Cosmogeracinae, Cosmogerax doesburgi Brindle, 1982. 


\section{dominicana Brindle, 1971 (Parasparatta)}

Parasparatta dominicana Brindle, 1971e: 14, figs 13-14.

\section{Type material}

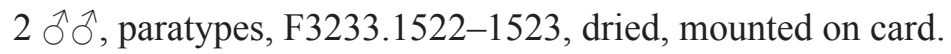

\section{Locality labels}

F3233.1522: Dominica / En Haut Jean / XII-7-1964 / P. J. Spangler.

F3233.1523: Dominica / Pont Casse. 1.7 / mi. e.12III65 / W. W. Wirth. // 65W66ex / decaying heart / Euterpe palm.

\section{Taxonomy}

Spongiphoridae, Sparattinae, Sparattini, Sparatta dominicana (Brindle, 1971).

\section{Remarks}

1. Transferred by Steinmann (1989b: 160).

2. Index card entry: West Indies.

3. Brindle (1971e: 16) states 'All the types are in the United States National Museum, except for one paratype male (Pont Casse) and one paratype female (En Haut Jean), in the Manchester Museum'; however, on the determination label for specimen F3233.1522, ' $O$ ' has been overwritten with ' $O$ ' so that both are males.

\section{dorsalis Brindle, 1969 (Acanthocordax)}

Fig. 30

Acanthocordax dorsalis Brindle, 1969e: 276, figs 1-2.

\section{Type material}

1 ठै, holotype, F3233.1279 (Fig. 30), dried, mounted on card;

\section{Type locality}

New Guinea / Aiyura / 6000 ft. / III.1945 / B. A. O’Connor // Imp. Inst. Ent. / Coll. No. 10635.

\section{Taxonomy}

Forficulidae, Opisthocosmiinae, Acanthocordax dorsalis Brindle, 1969.

\section{ecarinatus Brindle, 1973 (Esphalmenus)}

Esphalmenus ecarinatus Brindle, 1973f: 105, figs 133, 135-136.

\section{Type material}

1 J , paratype, F3233.1357; 1 \&, paratype, F3233.1358; dried, mounted on card.

\section{Locality labels}

F3233.1357-1358: S. Afr. Cape Prov. / Drakensbergen 8 miles / ENE Rhodes / 10.III.51 No. 223.

\section{Taxonomy}

Pygidicranidae, Esphalmeninae, Esphalmenus ecarinatus Brindle, 1973. 


\section{edentata Brindle, 1988 (Sarcinatrix)}

Fig. 33

Sarcinatrix edentata Brindle, 1988: 55, figs 3-5.

Type material

1 đ̆, holotype, F3233.1747 (Fig. 33); 1 , paratype, F3233.1748; dried, mounted on card.

\section{Type locality}

Panama, Fortuna / Wet forest, / at light, 1050m / 12 Sept. 1977 / Dr. Henk Wolda, STRI.

\section{Locality label}

F3233.1748: As type locality except date, 28 Nov. 1978.

\section{Taxonomy}

Forficulidae, Ancistrogastrinae, Sarcinatrix edentata Brindle, 1988.

\section{elongata Brindle, 1978 (Anisolabis)}

Anisolabis elongata Brindle, 1978: 103, figs 205-207, 278.
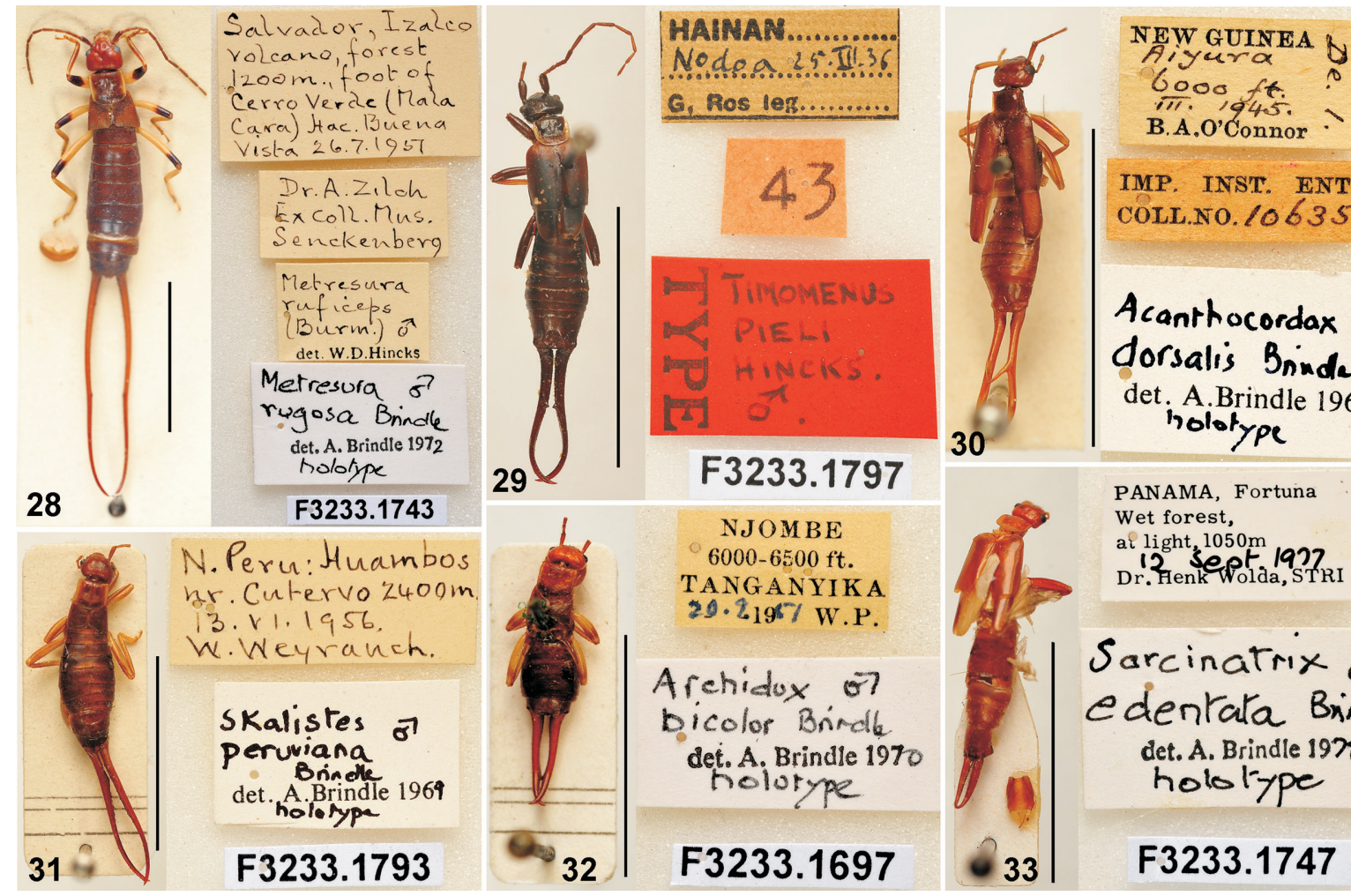

IMP. INST. ENT. COLL.NO. 10635

Acanthocordax of dorsalis Brinde det. A.Brindle 1969 holotype

PANAMA, Fortuna Wet forest,

ai light, $1050 \mathrm{~m}$ 19?7,

Sarcinatrix of edentata Bindle det. A. Brindle 1978 holotype

F3233.1747

Figs 28-33. Holotypes ( $\overbrace{}^{)}$of Forficulidae in the collection of the Manchester Museum. 28. Metresura rugosa Brindle, 1975. 29. Timomenus pieli Hincks, 1940. 30. Acanthocordax dorsalis Brindle, 1969 (F3233.1279). 31. Skalistes peruviana Brindle, 1970. 32. Archidux bicolor Brindle, 1973 (now Cosmiella bicolor). 33. Sarcinatrix edentata Brindle, 1988. Scale bars $=1 \mathrm{~cm}$. 


\section{Type material}

1 Oे, paratype, F3233.1376, dried, mounted on card. 1 \& , paratype, F3233.1377, dried, mounted on pin.

\section{Locality labels}

F3233.1376: Br. Cameroons / Sappo. nr. Buea / Mar. 1951; S. Tita.

F3233.1377: Br. Cameroons / Sasse-Sappo-Buea / Mar. 1952; S. Tita.

\section{Taxonomy}

Anisolabididae, Anisolabidinae, Anisolabis elongata Brindle, 1978.

\section{emarginata Brindle, 1977 (Marava)}

Marava emarginata Brindle, 1977b: 124, figs 30-32.

\section{Type material}

$1 \overbrace{}^{\lambda}$, paratype, F3233.1548, dried, mounted on card.

\section{Locality label}

Venezuela - Aragua / Rancho Grande / 1100 m 20-V-75.

\section{Taxonomy}

Spongiphoridae, Spongiphorinae, Marava emarginata Brindle, 1977.

\section{Remark}

The original description gives the date of the MMUE specimen as 29 Jan. 1967, not 20 May 1975 as on the specimen label. This date does not match any in the description.

\section{erythrocephala Brindle, 1964 (Gelotolabis)}

Gelotolabis erythrocephala Brindle, 1964: 753, figs 7, 14.

\section{Type material}

$1 \hat{\jmath}$, paratype, F3233.1378; dried, mounted on pin.

\section{Locality label}

Coll. Mus. Congo / Tanganyika Terr. : Kili- / manjaro, Vers. Sud. 4200- / 4600 m. 18/20-VII-1957.

\section{Taxonomy}

Anisolabididae, Anisolabidinae, Anisolabis felix felix Burr, 1907.

\section{Remark}

Gelotolabis erythrocephala was synonymised with Anisolabis felix felix Burr, 1907 by Brindle (1978: 127).

\section{excavatus Hincks, 1954 (Spongovostox)}

Spongovostox excavatus Hincks, 1954b: 119, figs 21-22. 
Type material

1 J, paratype, F3233.1608, dried, mounted on card.

\section{Locality label}

IFAN / Nimba (Guinée) / Lamotte et Roy / VII XII 51.

\section{Taxonomy}

Spongiphoridae, Spongiphorinae, Spongovostox excavatus Hincks, 1954.

\section{Remark}

Index card entry: Guinea, Nimba Mts.

\section{felix elgonensis Brindle, 1978 (Anisolabis)}

Anisolabis felix elgonensis Brindle, 1978: 128.

\section{Type material}

1 đે, paratype, F3233.1379, dried, mounted on card.

\section{Locality label}

Mount Elgon / in rotten tree / 16.I.1955 / leg. M. W. \& A. T.

\section{Taxonomy}

Anisolabididae, Anisolabidinae, Anisolabis felix elgonensis Brindle, 1978.

\section{flaviceps Brindle, 1975 (Metresura)}

Metresura flaviceps Brindle, 1975d: 85.

\section{Type material}

1 Sิ, paratype, F3233.1739, dried, mounted on pin.

\section{Locality label}

Costa Rica. / Aug. 1935. / EQ. A32140.

\section{Taxonomy}

Forficulidae, Neolobophorinae, Metresura flaviceps Brindle, 1975.

\section{flavicornis Hincks, 1947 (Cosmicula)}

Cosmicula flavicornis Hincks, 1947b: 40, figs 22-23.

\section{Type material}

1 ठे, paratype, F3233.1712, dried, mounted on card.

\section{Locality label}

N. E. Burma / Kambaiti, 1800 m / 11/6.1934 Malaise. 


\section{Taxonomy}

Forficulidae, Skendylinae, Cosmiella flavicornis (Hincks, 1947).

\section{Remark}

Transferred by Steinmann (1989c: 671).

\section{flavipes Brindle, 1975 (Metresura)}

Metresura flavipes Brindle, 1975d: 85.

\section{Type material}

1 S , paratype, F3233.1740, dried, mounted on pin.

\section{Locality label}

San Pedro de / Montes de Oca / C. R. 9-4-33 / C. H. Ballou.

\section{Taxonomy}

Forficulidae, Neolobophorinae, Metresura flavipes Brindle, 1975.

\section{Remarks}

1. Not listed in Hopkins et al. (2014).

2. The locality is given as Costa Rica in the original description.

\section{flavocincta Brindle, 1982 (Spongovostox)}

Spongovostox flavocincta Brindle, 1982a: 40, figs 16-17.

\section{Type material}

2 우, paratypes, F3233.1614-1615, dried, mounted on card.

\section{Locality labels}

F3233.1614: Panama, Fortuna / Wet forest, / at light, 1050m / 17 Sept. 1977 / Dr. Henk Wolda, STRI. F3233.1615: Same data except date 2 Aug. 1977.

\section{Taxonomy}

Spongiphoridae, Spongiphorinae, Spongovostox flavocinctus Brindle, 1982.

\section{Remark}

Changed to 'flavocinctus' by Steinmann (1989b: 347).

\section{flavohumeralis Brindle, 1988 (Marava)}

Fig. 45

Marava flavohumeralis Brindle, 1988: 52, fig. 16.

\section{Type material}

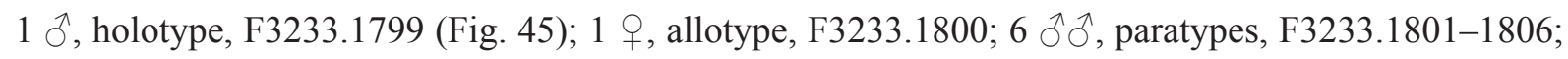

6 우, paratypes, F3233.1807-1812; dried, mounted on card. 
Type locality

Panama, Fortuna / Wet forest / at light, 1050m / 24.6.1978 / Dr. Henk Wolda, STRI.

\section{Locality labels}

As type locality except dates: F3233.1806, 11 Oct. 1976; F3233.1812, 11 May 1977; F3233.1810, 8 Jul. 1977; F3233.1805, 21 Aug. 1977; F3233.1808, F3233.1809, 24 Sep. 1977; F3233.1800-1802, 17 May 1978; F3233.1804, 10 Jun. 1978; F3233.1811, 9 Jul. 1978; F3233.1807, 25 Sep. 1978; F3233.1803, 14 Oct. 1978.

\section{Taxonomy}

Spongiphoridae, Spongiphorinae, Marava flavohumeralis Brindle, 1988.

\section{Remark}

The number of paratypes does not match that given in the original description, which states "Paratypes (all same locality): 8 §, 8 + , 11.x.1976 - 21.xi.1978 (MM)".

\section{formosanum Hincks, 1959 (Echinosoma)}

Echinosoma formosanum Hincks, 1959: 151, figs 138-139.

\section{Type material}

1 ऽે, paratype, F3233.1326: dried, mounted on card.

\section{Locality labels}

Tainan, / Formosa IV. // Echinosoma / formosanum / Hincks / Paratype $\widehat{o}$.

\section{Taxonomy}

Pygidicranidae, Echinosomatinae, Echinosoma formosanum Hincks, 1959.

\section{fulvescens Hincks, 1953 (Chaetospania)}

Chaetospania fulvescens Hincks, 1953: 373, figs 15-16.

\section{Type material}

2 우, paratypes, F3233.1510-1511, dried, mounted on card.

\section{Locality labels}

F3233.1510-1511: Madagascar CE / Perinet (parapluie) / XI.1946 J. Millot.

\section{Taxonomy}

Spongiphoridae, Sparattinae, Chaetospaniini, Chaetospania fulvescens Hincks, 1953.

\section{fusca Brindle, 1966 (Brachylabis)}

Brachylabis fusca Brindle, 1966e: 39, fig. 13.

\section{Type material}

1 ô, paratype, F3233.1470; 1 , paratype, F3233.1471; dried, mounted on card. 


\section{Locality labels}

F3233.1470: Forêt transition // Col. Mus. Congo / Tanganyika Terr.: Uluguru / Mts., vall Ululu-Ndogo, / 1500 m. 8-V-1957.

F3233.1471: Forêt de montagne // Col. Mus. Congo / Tanganyika Terr.: Uluguru / Mts., sommet du Kidunda / 1800-1950 m. 3-V-1957.

\section{Taxonomy}

Anisolabididae, Antisolabidinae, Antisolabis fusca (Brindle, 1966).

\section{Remarks}

1. Transferred by Steinmann (1989a: 344).

2. In the original description only one set of locality data is given, as for F3233.1470.

\section{fuscata clavata Brindle, 1972 (Chaetospania)}

Chaetospania fuscata clavata Brindle, 1972c: 148, figs 13e-13f, 13j.

\section{Type material}

$2 \widehat{\jmath}$, paratypes, F3233.602-603; 1 q, paratype, F3233.604: dried, mounted on card.

\section{Locality labels}

F3233.602-604: Pilgo River / Guam / 26 May 1945 // G. E. Bohart / J. C. Gressit.

\section{Taxonomy}

Spongiphoridae, Sparattinae, Chaetospaniini, Chaetospania fuscata clavata Brindle, 1972.

\section{fuscata fuscata Brindle, 1972 (Chaetospania)}

Chaetospania fuscata fuscata Brindle, 1972c: 146, figs 13c-13d, 13k.

\section{Type material}

1 ô, paratype, F3233.599; 1 +, paratype, F3233.600; dried, mounted on card.

\section{Locality labels}

F3233.599-600: Kusaie. 580m / Mt. Matante / 11 Feb. 1953 / J. F. G. Clarke.

\section{Taxonomy}

Spongiphoridae, Sparattinae, Chaetospaniini, Chaetospania fuscata fuscata Brindle, 1972.

\section{Remark}

Index card entry: Indonesia.

\section{fuscula Brindle, 1970 (Brachylabis)}

Brachylabis fuscula Brindle, 1970d: 15, figs 3-4, 6.

\section{Type material}

1 , paratype, F3233.1472, dried, mounted on card. 


\section{Locality label}

Belinga / 109T / H. Coiffait // Mission Biologique / Au Gabon / P.P. Grasse Directeur.

\section{Taxonomy}

Anisolabididae, Brachylabidinae, Brachylabis fuscula Brindle, 1970.

\section{gariazzoi var. tanganyikae Hincks, 1957 (Forcipula)}

Forcipula gariazzoi var. tanganyikae Hincks, 1957a: 42.

\section{Type material}

1 ô, paratype, F3233.1492; 1 q, paratype, F3233.1493; dried, mounted on pin.

\section{Locality labels}

F3233.1492: Baie de Kasoje / Riv. Lubulungu / 15.XII.46 / Miss. Tanganika 18.

F3233.1493: Same data except date 15 Dec. 1946.

\section{Taxonomy}

Labiduridae, Labidurinae, Forcipula tanganyikae Hincks, 1957.

\section{Remarks}

1. Transferred by Steinmann (1980: 235).

2. The original description has 'gariazzoi', not 'gariazzi' as in Borelli's original description of Forcipula gariazzi (Borelli, 1900: 1).

3. Index card entry: Tanzania.

\section{gnathonica Brindle, 1970 (Chaetospania)}

Chaetospania gnathonica Brindle, 1970f: 673, figs 28-29.

\section{Type material}

1 Oे, paratype, F3233.597, dried, mounted on card.

\section{Locality label}

Forest litter / Solomon Is. / Kolombangara / Nr. Kuzi / 3-9.ix.1968 / P.N. Lawrence / Roy. Soc. Exp. / B. M. 166-1.

\section{Taxonomy}

Spongiphoridae, Sparattinae, Chaetospaniini, Chaetospania gnathonica Brindle, 1970.

\section{Remark}

In the original description, the paratype at MMUE is stated to be a female. Brindle's determination label states $\hat{\jmath}$.

\section{gracilis Brindle, 1966 (Allostethus)}

Allostethus gracilis Brindle, 1966h: 442, fig. 6. 


\section{Type material}

1 ô, paratype, F3233.298, dried, mounted on card.

\section{Locality label}

Momungan / Mindanao.

\section{Taxonomy}

Labiduridae, Allostethinae, Allostethus gracilis Brindle, 1966.

\section{Remarks}

1. The original description gives the locality as Philippine Islands.

2. Steinmann (1989a: 412) has the taxon as Allostethus gracile.

\section{gracilis Brindle, 1988 (Marava)}

Fig. 47

Marava gracilis Brindle, 1988: 52, figs 20-21.

\section{Type material}

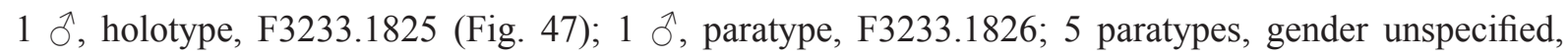
F3233.1827-1831; dried, mounted on card.

\section{Type locality}

Panama, Barro / Colorado Is., S-M / light trap, ground / level 8.11.1978 / Dr. Henk Wolda, STRI.

\section{Locality labels}

As for type except date: F3233.1827, 4 Nov. 1978; F3233.1828, 7 Nov. 1978; F3233.1829, 9 Nov. 1978; F3233.1830, 13 Nov. 1978; F3233.1826, 20 Nov. 1978; F3233.1831, 4 Dec. 1978.

\section{Taxonomy}

Spongiphoridae, Spongiphorinae, Marava gracilis Brindle, 1988.

\section{Remarks}

1. Not listed in Hopkins et al. (2014).

2. The original description refers to three male and four female paratypes at MMUE (i.e., one more than listed here) with the date range, 15 Jul. 1978-4 Dec. 1978.

\section{grandicollis Hincks, 1954 (Antisolabis)}

Antisolabis grandicollis Hincks, 1954b: 117, figs 19-20.

\section{Type material}

1 \&, paratype, F3233.1473, dried, mounted on card.

\section{Locality label}

IFAN / Nimba (Guinée) / Lamotte et Roy / VII. XII. 51.

\section{Taxonomy}

Anisolabididae, Isolabidinae, Isolabis grandicollis (Hincks, 1954). 


\section{Remarks}

1. Transferred by Brindle (1978: 46).

2. Index card entry: Nimba Mts.

greensladei Brindle, 1970 (Brachylabis)

Brachylabis greensladei Brindle, 1970f: 659, figs 17-18.

\section{Type material}

1 Oे, paratype, F3233.112; 1 क, paratype, F3233.105; dried, mounted on card.

\section{Locality labels}

F3233.112, F3233.105: Solomon Is. / Guadacanal [sic] / Mt. Austen / 24.VIII.1965 / P. Greenslade.

\section{Taxonomy}

Anisolabididae, Antisolabidinae, Antisolabis greensladei (Brindle, 1970).

\section{Remarks}

1. Transferred by Steinmann (1989a: 359).

2. The original description states that only 1 male paratype was passed to MMUE. F3233.112 bears Brindle's 1970 determination label stating 'Paratype'.

3. The locality, spelled 'Guadacanal' on the locality label, is given as 'Guadalcanal' in the description.

\section{griveaudi Brindle, 1966 (Marava)}

Marava griveaudi Brindle, 1966g: 248, figs 47-48, 55.

\section{Type material}

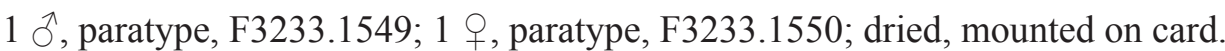

\section{Locality labels}

F3233.1549: Sakarana / Lambomakandro / A R.

F3233.1550: Sakarana / Lambomakandro / P. Griveaud.

\section{Taxonomy}

Spongiphoridae, Spongiphorinae, Marava griveaudi Brindle, 1966.

\section{guatemalensis Brindle, 1973 (Geracides)}

Geracides guatemalensis Brindle, 1973a: 154, figs 1-4.

\section{Type material}

1 Oे, paratype, F3233.1530, dried, mounted on card.

\section{Locality label}

Guatemala / 394347 / Primavera logs / N. York / Mar. 291939.

\section{Taxonomy}

Spongiphoridae, Cosmogeracinae, Cosmogerax guatemalensis (Brindle, 1973). 


\section{Remarks}

Taxonomy follows Steinmann 1989c. Transferred by Brindle (1982a: 37) as Cosmogerax guatemalensis. Steinmann (1989b: 104) has C. guatemalense. Hopkins et al. (2014) have C. guatemalensis.

\section{gurneyi Brindle, 1970 (Setocordax)}

Setocordax gurneyi Brindle, 1970a: 99, fig. 1.

\section{Type material}

1 O , paratype, F3233.1754, dried, mounted on pin.

\section{Locality label}

Colombia / with orchids / at Miami, Fla / Aug. 7, 1963 / 6318995.

\section{Taxonomy}

Forficulidae, Neolobophorinae, Setocordax gurneyi Brindle, 1970.

\section{hainanensis Hincks, 1940 (Diplatys)}

Fig. 27

Diplatys hainanensis Hincks, 1940: 30, figs 1-3.

\section{Type material}

1 ठ , holotype F3233.1298 (Fig. 27), dried, mounted on card.

\section{Type locality}

Hainan, Sama / 18.VII.36 / G. Ros. leg.

\section{Taxonomy}

Diplatyidae, Diplatyinae, Paradiplatys salvazae (Burr, 1917).

\section{Remarks}

Hincks (1955a: 94) synonymised Diplatys hainanensis Hincks, 1940 with D. salvazae Burr, 1917. Transferred to Lobodiplatys (Paradiplatys) salvazae (Burr, 1917) by Steinmann (1986a: 178). The combination Paradiplatys (Paradiplatys) salvazae (Burr) was reinstated by Engel \& Haas (2007: 20).

\section{halli Hincks, 1949 (Nesogaster)}

Nesogaster halli Hincks, 1949b: 202, figs 1-3.

\section{Type material}

1 ô, paratype, F3233.541; 1 q, paratype, F3233.542; dried, mounted on card.

\section{Locality labels}

F3233.541: In house. / Titirangi / March 1944 / M. W. Carter.

F3233.542: Sieved. / Mt. Albert. / Auckland. / 24th Sep. 1941. / D. Spiller. Coll.

\section{Taxonomy}

Spongiphoridae, Nesogastrinae, Nesogaster halli Hincks, 1949. 


\section{Remarks}

1. Index card entry: New Zealand.

2. Data in the original description which matches that for specimen F3233.542 has the date 29 Sep. 1941, not 24 Sep. 1941.

\section{hamata Brindle, 1978 (Anisolabis)}

Anisolabis hamata Brindle, 1978: 132, figs 201-204, 272.

\section{Type material}

1 q, paratype, F3233.1380, dried, mounted on card.

\section{Locality label}

Dinsha, Bale / Ethiopia / 1971 / G. De Rougemont.

\section{Taxonomy}

Anisolabididae, Anisolabidinae, Flexiolabis hamata (Brindle, 1978).

\section{Remark}

Transferred by Steinmann (1989a: 36).

\section{hamatus Brindle, 1972 (Diplatys)}

Diplatys hamatus Brindle, 1972a: 281, figs 1-2.

\section{Type material}

$1 \hat{\jmath}$, paratype, F3233.1301, dried, mounted on card.

\section{Locality label}

Costa Rica / Turrialba / VII-15-19-65 / P. J. Spangler.

\section{Taxonomy}

Diplatyidae, Diplatyinae, Haplodiplatys hamatus (Brindle, 1972).

\section{Remark}

Transferred by Steinmann (1974: 201).

\section{hebardi Hincks, 1957 (Echinosoma)}

Echinosoma hebardi Hincks, 1957b: 55, figs 17-18.

\section{Type material}

1 ô, paratype, F3233.1327, dried, mounted on card.

\section{Locality label}

Tanganyika / Southern Prov. / Nachingwea / 3.XI.53 - III.54 / V. F. Eastop.

\section{Taxonomy}

Pygidicranidae, Echinosomatinae, Echinosoma hebardi Hincks, 1957. 


\section{heliconia Brindle, 1985 (Labia)}

Labia heliconia Brindle, 1985: 79, figs 10, 15, 22.

\section{Type material}

1 đ , paratype, F3233.1545; 1 + , paratype, F3233.1546; dried, mounted on card.

\section{Locality labels}

F3233.1545: Ecuador: Prov. Napo / Lago Agrio. in / Heliconia 26.9.1975 / Andrea Langley.

F3233.1546: Same data except date 23 Aug. 1975.

\section{Taxonomy}

Spongiphoridae, Labiinae, Paralabella heliconia (Brindle, 1985).

\section{Remark}

Transferred by Steinmann (1989c: 583).

\section{hincksi Ramamurthi, 1960 (Auchenomus)}

Auchenomus hincksi Ramamurthi, 1960: 35, figs 2-3.

\section{Type material}

$1 \widehat{\partial}, 1$, paratypes, F3233.1500, dried, mounted on card.

\section{Locality label}

India: Madras / B. N. Ramamurthi / (ô gen. Removed)

\section{Taxonomy}

Spongiphoridae, Sparattinae, Auchenomini, Auchenomus hincksi Ramamurthi, 1960.

\section{hincksi Brindle, 1964 (Gelotolabis)}

Fig. 19

Gelotolabis hincksi Brindle, 1964: 753, fig. 10.

\section{Type material}

1 Oे, holotype, F3233.1381 (Fig. 19); 1 ○, allotype, F3233.1382; dried, mounted on pin.

\section{Type locality}

South Kulal, / Aug. 1944 / J. Adamson.

\section{Locality label}

F3233.1382: As type locality.

\section{Taxonomy}

Anisolabididae, Anisolabidinae, Gonolabis hincksi (Brindle, 1964).

\section{Remark}

Transferred by Steinmann (1978: 196). 


\section{hincksi Brindle, 1970 (Pseudovostox)}

Fig. 54

Pseudovostox hincksi Brindle, 1970e: 259, figs 1-2, 10.

\section{Type material}

1 Oे, holotype, F3233.1593 (Fig. 54), dried, mounted on card.

\section{Type locality}

V. Falls Rd. 148 / S. Rhodesia m. // XI. 1952 / Nat. Museum / S. Rhodesia.

\section{Taxonomy}

Spongiphoridae, Geracinae, Pseudovostox hincksi Brindle, 1970.

\section{hincksi Brindle, 1966 (Vandex)}

Vandex hincksi Brindle, 1966c: 44, figs 5-6, 9.

\section{Type material}

$3 \widehat{\partial} \widehat{\jmath}$, paratypes, F3233.1624-1626; 2 우, paratypes, F3233.1627-1628; dried, mounted on card.

\section{Locality labels}

F3233.1624: Coll. Mus. Congo / Ruanda : Rutovu, for. du / Rugege, 2350 m. / P. Basilewsky 20/23-I-53. F3233.1625-1627: Récolté dans / l'humus / (forêt Hagenia). // I.R.S.A.C.-Mus. Congo / Kivu : T. Kabare, contr. / S. E. du Kahuzi, / 13-VIII-1951 / N. Leleup.

F3233.1628: Récolté dans / l'humus. // I.R.S.A.C.- Mus. Congo / Kivu : Terr. Lubero / 2.200 m. 27-XI1951 / N. Leleup / (for. mont. avec bamb.).

\section{Taxonomy}

Spongiphoridae, Vandicinae, Vandex hincksi Brindle, 1966.

\section{Remarks}

1. Index card entry: Africa, Zaire, Ruanda, Burundi borders, Kivu Province.

2. With regard to the locality of F3233.1624, Brindle (1966c: 46) states 'This species was incorrectly recorded by the late Dr. W.D. Hincks in 1955 as V. schubotzi, on a series from the Rugege Forest, Ruanda. Only a pair from this series is now available, but these clearly belong to the present species.'

\section{humicola Brindle, 1966 (Brachylabis)}

Brachylabis humicola Brindle, 1966e: 37, figs 11, 19.

\section{Type material}

1 §, paratype, F3233.1366; 1 + , paratype, F3233.1367; dried, mounted on card.

\section{Locality labels}

F3233.1366-1367: I.R.S.A.C.-Mus. Congo / Kivu: Terr. Kabare, S. E. / Kahuzi, 2080/2200 m. / N. Leleup VIII-1951. 


\section{Taxonomy}

Anisolabididae, Isolabidinae, Africolabis humicola (Brindle, 1966).

\section{Remark}

Transferred by Brindle (1978: 25).

\section{inca Brindle, 1970 (Neolobophora)}

Neolobophora inca Brindle, 1970a: 102, fig. 11.

\section{Type material}

1 §, paratype, F3233.1752; 1 \%, paratype, F3233.1753; dried, mounted on card.

\section{Locality labels}

F3233.1752: Torentoy Canyon / Base Machu. Picchu / 2000-2200m. Peru / VI-VII. 1964. Malkin. F3233.1753: Machu-Picchu (sobre / ruinas, 2600-2800m) / Peru - 1-2.VII.1964 / B. Malkin.

\section{Taxonomy}

Forficulidae, Neolobophorinae, Neolobophora inca Brindle, 1970.

\section{incisa Brindle, 1970 (Adiathella)}

Adiathella incisa Brindle, 1970f: 690, figs 59-60.

\section{Type material}

1 Oे, paratype, F3233.924; 1 +, paratype, F3233.925; dried, mounted on card.

\section{Locality labels}

F3233.924: Guadacanal [sic] / Papamanaisiu / 5700' / 8-XI-1965 / R. S. Exp.

F3233.925: Forest litter / Solomon Is. / Guadalcanal / Popamanisiu / c 7000'. // 6 - 8. XI. 1965 / P. N. Lawrence / Roy. Soc. Exped. / Brit. Mus. 1966-I.

\section{Taxonomy}

Chelisochidae, Chelisochinae, Adiathella incisa Brindle, 1970.

\section{Remarks}

1. It is unclear whether F3233.925 is the allotype referred to in the original description, which only refers to one male paratype at MMUE. The allotype locality data in the description matches F3233.925.

2. The locality 'Guadacanal' on the label for F3233.925 is given as 'Guadalcanal' in the description.

\section{incisiodes Brindle, 1978 (Anisolabis)}

Anisolabis incisiodes Brindle, 1978: 94, figs 136-138, 247.

\section{Type material}

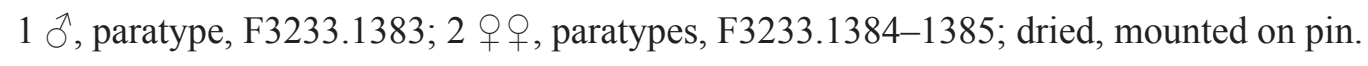




\section{Locality labels}

F3233.1383: Museum Paris / Congo Belge / Eala III.1935 / J. Ghesquière.

F3233.1384: Museum Paris / Congo Belge / Eala I.1935 / J. Ghesquière.

F3233.1385: Musée du Congo / Eala / - VIII -1935 / J. Ghesquière.

\section{Taxonomy}

Anisolabididae, Anisolabidinae, Anisolabis incisiodes Brindle, 1978.

\section{Remark}

The spelling 'incisiodes' is used consistently in the original description. Steinmann (1989c: 217) and Hopkins et al. (2014) refer to this species as incisoides.

\section{indica Brindle, 1966 (Forcipula)}

Forcipula indica Brindle, 1966d: 265, figs 26, 36.

\section{Type material}

1 ô, paratype, F3233.1494, dried, mounted on pin.

\section{Locality label}

India: U.P. / Song River / Dehra Dun. // Under stone / in water / 17.6.1945.

\section{Taxonomy}

Labiduridae, Labidurinae, Forcipula indica Brindle, 1966.

\section{Remark}

The date given in the original description is 1948 , not 1945 as on the specimen label.

\section{insulana Brindle, 1972 (Gonolabis)}

Gonolabis insulana Brindle, 1972c: 119, fig. 5a-e.

\section{Type material}

$2 \widehat{\jmath}$, paratypes, F3233.188, F3233.190; 1 ค , paratype, F3233.189; dried, mounted on pin.

\section{Locality labels}

F3233.188: S. / MapId. Yap Group / Jul-Au 50 / R J Goss. Pac. S. Bd. F3233.189: Pac. S. Bd. Yap Group / Jul-Au 50 / R J Goss. Gagil Dist. F3233.190: N. Yap Is. / Micronesia / July-Aug. 1950 / R. J. Goss

\section{Taxonomy}

Anisolabididae, Anisolabidinae, Gonolabis insulana Brindle, 1972.

\section{insularis Brindle, 1976 (Auchenomus)}

Auchenomus insularis Brindle, 1976c: 228, figs 27-28.

\section{Type material}

1 , paratype, F3233.561, dried, mounted on card. 


\section{Locality label}

New Hebrides / Espiritu Santo I. / (SW): Above / Namatasopa, 400m. / Aug. 30, 1957.

\section{Taxonomy}

Spongiphoridae, Sparattinae, Auchenomini, Auchenomus insularis Brindle, 1976.

\section{intermedius Brindle, 1966 (Archidux)}

Archidux intermedius Brindle, 1966c: 54, figs 13, 17, 21, 24.

\section{Type material}

$1 \widehat{\jmath}$, paratype, F3233.1702; 1 \&, paratype, F3233.1703; dried, mounted on card.

\section{Locality labels}

F3233.1702: I.R.S.A.C.-Mus.-Congo / Kivu: Mont Muhi, ruiss. / Isale, 3025 m. (humus) / N. Leleup VII-1955.

F3233.1703: Forêt transition // I.R.S.A.C.-Mus. Congo / Kivu : Riv. Nyakagera, terr. / Kabare 1600/1700 m. / N. Leleup XI-1955.

\section{Taxonomy}

Forficulidae, Skendylinae, Cosmiella intermedia (Brindle, 1966).

\section{Remarks}

1. Transferred by Steinmann (1989c: 672).

2. Index card entry: Rwanda, Lake Kivu.

3. In the original description the specimen with data which matches F3233.1702 is a female paratype, not a male.

\section{jacobsoni Burr, 1912 (Arixenia)}

Fig. 16

Arixenia jacobsoni Burr, 1912: 105, fig.

\section{Type material}

2 syntypes, gender not specified, F3233.1866-67 (Fig. 16); 1 ふૈ, syntype, F3233.1868.

\section{Locality labels}

F3233.1866: E. Jacobson / Babakan. (Ban- / joemas) / Java / Mrt 1911 / Gouwa Lawa (grot) // Cotype // 3550 // A. jacobsoni Burr / det. Burr // Ex Mus. Tring / via Dr. K. Jordan.

F3233.1867: As F3233.1866 except number 3551.

F3233.1868: As F3233.1866 except number 3547 and det. label: Arixenia jacobsoni / Burr $\widehat{O}$ / det. Burr.

\section{Taxonomy}

Arixeniidae, Xeniaria jacobsoni (Burr, 1912).

\section{Remarks}

1. Transferred by Maa (Nakata \& Maa 1974: 319). 
2. In the original description, the author refers to the 'nomotype' being in his collection and to 'numerous specimens: Edw. Jacobson.'

3. In the original description, the locality is given as Banjoumas Residency, Gouwa Lawa cave near the sea-shore at Babakan, Java.

jacobsoni Borelli, 1927 (Timomenus)

Timomenus jacobsoni Borelli, 1927: 78, fig. 10.

\section{Type material}

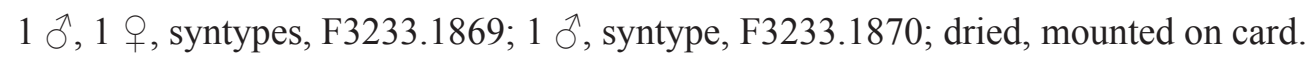

\section{Locality labels}

F3233.1869: Fort de Kock / (Sumatra) 920. M. / 1925 / leg. E. Jacobson.

F3233.1870: Anei Kloof / (Sumatra's West / Kust) 500 M 1925 / leg. E. Jacobson.

\section{Taxonomy}

Forficulidae, Opisthocosmiinae, Timomenus jacobsoni Borelli, 1927.

\section{Remarks}

Brindle (1968f: 246) considered T. jacobsoni to be a lighter form of T. bicuspis (Stål, 1860); however, Steinmann (1982b) presents T. jacobsoni as a species in its own right. In the original description, Borelli referred to 4 specimens examined: $1 \delta$ with data as F3233.1870, and $2 \lambda \hat{\delta}$ and $1 \%$ with data as F3233.1869 except the date 1924. He did not designate a holotype and it appears therefore that all four specimens should be considered syntypes. According to Steinmann (1982b: 365), 1 ते was held at Museo ed Istituto di Zoologia Sistematoca, Torino, which Steinmann appears to have designated the holotype ('without penis', although the male genitalia are figured in Steinmann's description).

\section{javana Hincks, 1955 (Cranopygia)}

Cranopygia javana Hincks, 1955c: 810, fig. 3.

\section{Type material}

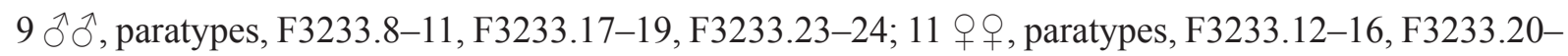
22, F3233.25-27; dried, mounted on pin.

\section{Locality labels}

F3233.18-21: E. Java / Soember / Brantas / Mt. Ardjuno / 6000' 2.37 / M. E. Walsh. F3233.15, F3233.17, F3233.22-24, F3233.27: Java: / Soember / Brantas / II.37 M. E. Walsh. F3233.8-10, F3233.13-14, F3233.16, F3233.25: Kangean I. / (E. of Madura) / Ardjasa / M. E. Walsh. F3233.11-12, F3233.26: Kangean I. / (E. of Madura)/ Ardjasa 50' / 5.36 M. E. Walsh.

\section{Taxonomy}

Pygidicranidae, Pygidicraninae, Cranopygia javana Hincks, 1955.

\section{javanicus Hincks, 1951 (Nesogaster)}

Fig. 58

Nesogaster javanicus Hincks, 1951b: 570, figs 12-14. 


\section{Type material}

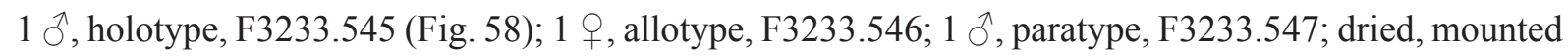
on card.

\section{Type locality}

East Java: / Soember Brantas / Feb. 1937 / M. E. Walsh.

\section{Locality labels}

F3233.546-547: As for type locality.

\section{Taxonomy}

Spongiphoridae, Nesogastrinae, Nesogaster aculeatus (de Bormans, 1900).

\section{Remark}

Nesogaster javanicus Hincks, 1951 synonymised with N. aculeatus (de Bormans, 1900) by Steinmann (1989b: 23).

\section{kambaitiensis Hincks, 1947 (Forficula)}

Forficula kambaitiensis Hincks, 1947b: 29, figs 15-16.

\section{Type material}

1 Oे, paratype, F3233.1729; 1 + , paratype, F3233.1730; dried, mounted on card.

\section{Locality labels}

F3233.1729: N. E. Burma / Kambaiti, 7000 ft / 19/4 R. Malaise.

F3233.1730: N. E. Burma / Kambaiti, 2000 m / 6/4. 1934 Malaise.

\section{Taxonomy}

Forficulidae, Forficulinae, Forficula kambaitiensis Hincks, 1947.

\section{kelangi Brindle, 1969 (Brachylabis)}

Brachylabis kelangi Brindle, 1969b: 199, figs 1-3.

\section{Type material}

1 ऽ, paratype, F3233.1423, dried, mounted on card.

\section{Locality label}

Kapar Mangrove / Forest Reserve / Kelang, West / Malaysia. 7.2.1969 / A. Sase Kumar.

\section{Taxonomy}

Anisolabididae, Antisolabidinae, Antisolabis kelangi (Brindle, 1969).

\section{Remarks}

1. Transferred by Steinmann (1989a: 370).

2. Index card entry: Port Swettenham. 


\section{kermadecensis Giles, 1973 (Labia)}

Labia kermadecensis Giles, 1973: 306, figs 1-4.

\section{Type material}

1 ô, paratype, F3233.670, dried, mounted on card.

\section{Locality label}

Bells Flat, Raoul Isl, / Kermadec Isls. / 3 January 1967, J. C. Watt leg. / litter sample 67/57.

\section{Taxonomy}

Spongiphoridae, Labiinae, Spirolabia kermadecensis (Giles, 1973).

\section{Remarks}

1. Transferred by Steinmann (1987: 181).

2. Additional locality data in the original description: '... litter under Metrosideros kermadecensis, Myoporum laetum and Rhopalostylus, dry litter on 'Oneraki black sandy silt', sieved, sample U ...' (Giles 1973: 308).

\section{kivuensis Brindle, 1973 (Chaetospania)}

Chaetospania kivuensis Brindle, 1973f: 155, figs 191-194.

\section{Type material}

1 , paratype, F3233.1512, dried, mounted on card.

\section{Locality label}

Récolté sous / écorce d'arbre mort // I.R.S.A.C. - Mus. Congo / Kivu: Terr. Mwenga, / S.0. Itombwe, Luiko / 2050 m (for. mont.) I-1952 / N. Leleup.

\section{Taxonomy}

Spongiphoridae, Sparattinae, Chaetospaniini, Chaetospania kivuensis Brindle, 1973.

\section{Remark}

Index card entry: Africa, Zaire, Kivu.

\section{kjellanderi Brindle, 1967 (Diplatys)}

Diplatys kjellanderi Brindle, 1967b: 148, figs 3, 6, 8 .

\section{Type material}

1 ठ , paratype, F3233.1302, dried, mounted on card.

\section{Locality label}

Bulbalan, Luzon.

\section{Taxonomy}

Diplatyidae, Diplatyinae, Circodiplatys kjellanderi (Brindle, 1967). 


\section{Remarks}

Transferred by Steinmann (1986b: 109). Steinmann (1989c: 31) and Hopkins et al. (2014) have the spelling 'kjellenderi'. Brindle dedicated this species to Dr. E. Kjellander (Brindle 1967b: 150).

\section{koepckei Brindle, 1966 (Idolopsalis)}

Fig. 25

Idolopsalis koepckei Brindle, 1966a: 145, figs 3, 7, 14.

\section{Type material}

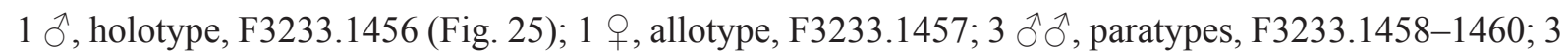

우, paratypes, F3233.1461-1463; dried, mounted on card.

\section{Type locality}

Hda. Taulis Peru / (ca. 6 50’ S, 79¹0’ W) / 27 Dezember 1952 / leg.: Koepcke.

\section{Locality labels}

F3233.1457: As type locality.

F3233.1458: N. Peru, w. slopes /Andes, Hacienda / Taulis ca 6' 50" / 79' 10" S. Ca 2700 m. / bergurwald. / 27.12.52. Koepcke.

F3233.1459-1462: Same data except 1700m. / montane forest / rotten wood / leaves on ground / H W Koepcke 1.52. // *Kp. 403j.

F3233.1463: As type locality except mid. Jan. 1952.

\section{Taxonomy}

Anisolabididae, Idolopsalidinae, Idolopsalis koepckei Brindle, 1966.

\section{Remark}

The original description gives the date for the holotype and the specimen matching F3233.1458 as 24 Dec. 1952, not 27 Dec. 1952.

\section{koepckei Brindle, 1968 (Strongylopsalis)}

Fig. 55

Strongylopsalis koepckei Brindle, 1968c: 279, figs 1, 4.

\section{Type material}

1 đે, holotype, F3233.1619 (Fig. 55); 1 + , allotype, F3233.1620; dried, mounted on card.

\section{Type locality}

Peru / Bei Kampañillayo / (bei Tarma) 6 August 1953 / leg. Koepcke.

\section{Locality label}

F3233.1620: bei Campanillay / Peru (bei Tarna) / 6.viii.1953 / H. W. Koepcke.

\section{Taxonomy}

Spongiphoridae, Strongylopsalinae, Strongylopsalis koepckei Brindle, 1968. 


\section{Remark}

The original description gives the locality as Campanillayo (bei Tarna). The index card has Bei Campanillayo. (Tarma is a city in Peru.)

\section{kuscheli Hincks, 1959 (Esphalmenus)}

Fig. 35

Esphalmenus kuscheli Hincks, 1959: 201, fig. 204.

\section{Type material}

1 Oे, holotype, F3233.1359 (Fig. 35); 1 q, allotype, F3233.1360; dried, mounted on card.

\section{Type locality}

Chile. Bio-Bio / Laguna Laja / Los Barros - 2100 m / 18.I.48. // Coll. / Kuschel // Esphalmenus / Kuscheli / Hincks / Holotype $\widehat{\sigma} /$ det. W. D. Hincks.

\section{Locality label}

F3233.1360: Chile. Bio-Bio / Laguna Laja / Los Barros - 1500 m / 23.I.48. // Esphalmenus / Kuscheli / Hincks / Allotype $q$ / det. W. D. Hincks.

\section{Taxonomy}

Pygidicranidae, Esphalmeninae, Esphalmenus kuscheli Hincks, 1959.

\section{labioides Hincks, 1952 (Geracodes)}

Geracodes labioides Hincks, 1952b: 23, figs 7-9.

\section{Type material}

1 ภे, paratype, F3233.1445; 5 q $\odot$, paratypes, F3233.1446-1450; dried, mounted on card.

\section{Locality labels}

F3233.1445-1448: Sierra Leone / Njala / 15.ix - 8.xii 1930 / E. Hargreaves / At light. F3233.1449: Sierra Leone / Njala / 30.X.32;

F3233.1450: Same data except date 21 Nov. 1923.

\section{Taxonomy}

Anisolabididae, Isolabidinae, Geracodes labioides Hincks, 1952.

\section{laevigata Brindle, 1978 (Anisolabis)}

Anisolabis laevigata Brindle, 1978: 100, figs 142, 145, 251.

\section{Type material}

1 , paratype, F3233.1386, dried, mounted on card.

\section{Locality label}

Nimba (Guinée) / Lamotte, Amiet, / Vanderplaetsen / XII 56 - V 57. 


\section{Taxonomy}

Anisolabididae, Anisolabidinae, Gonolabis laevigata (Brindle, 1978).

\section{Remark}

Transferred by Steinman (1989a: 197).

\section{laevis Brindle, 1975 (Archidux)}

Archidux laevis Brindle, 1975b: 694, fig. 4.

\section{Type material}

2 ふึ, paratypes, F3233.1704-1705, dried, mounted on card.

\section{Locality labels}

F3233.1704-1705: Tanzanie: Mts Uluguru / Chenzema, alt. 1700m. / 2-22/VII/71 // Coll. Mus. Tervuren / Mission Mts Uluguru / L. Berger, N. Leleup / J. Debecker V/VIII/71.

\section{Taxonomy}

Forficulidae, Skendylinae, Cosmiella laevis (Brindle, 1975).

\section{Remarks}

1. Transferred by Steinmann (1989c: 672).

2. The original description refers to only one male paratype at MMUE.

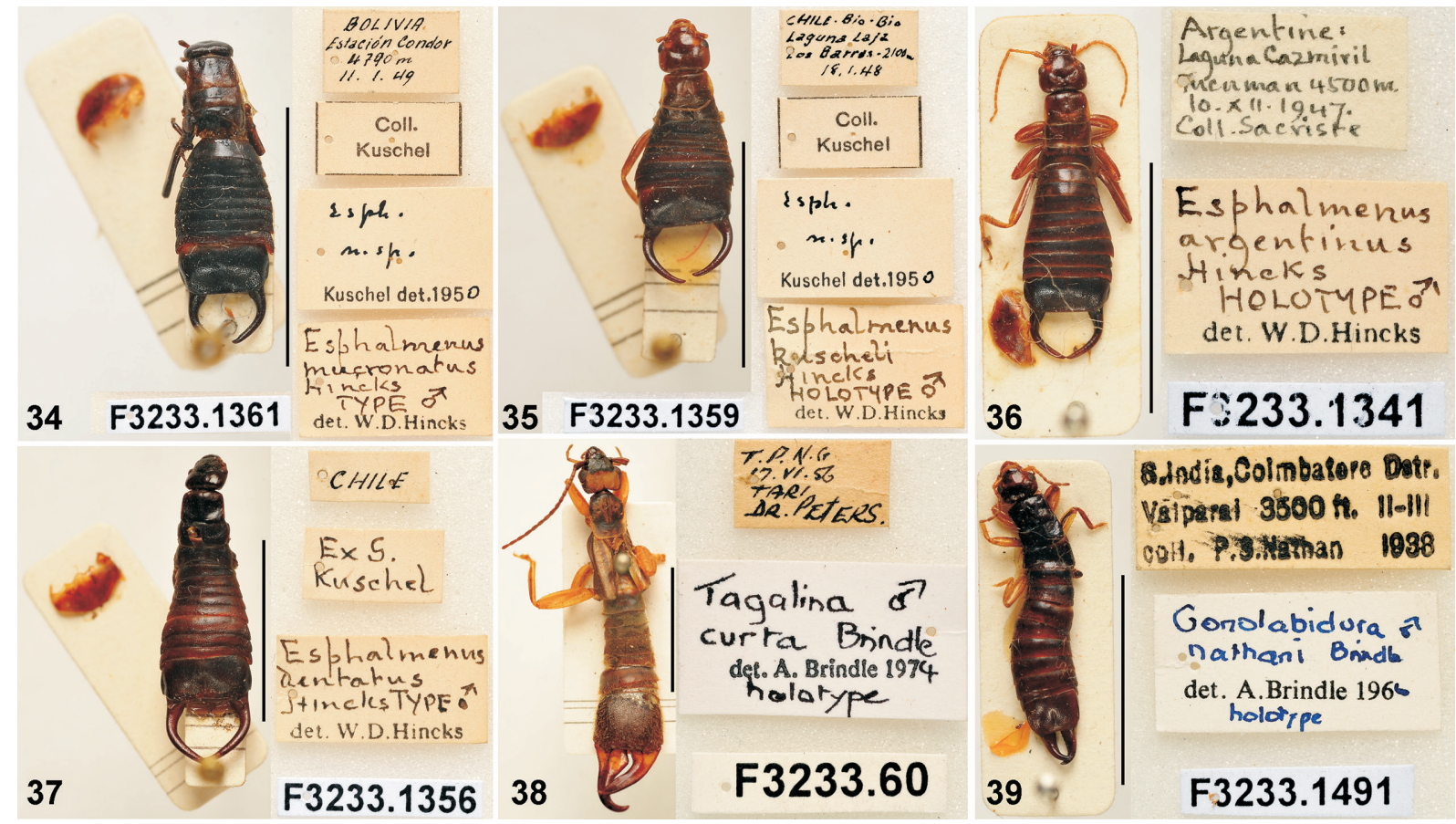

Figs 34-39. Holotypes $(ð)$ of Pygidicranidae and Labiduridae in the collection of the Manchester Museum. 34. Esphalmenus mucronatus Hincks, 1959. 35. E. kuscheli Hincks, 1959. 36. E. argentinus Hincks, 1959. 37. E. dentatus Hincks, 1959. 38. Tagalina curta Brindle, 1975. 39. Gonolabidura nathani Brindle, 1965. Scale bars $=1 \mathrm{~cm}$. 
laminata Brindle, 1973 (Strongylopsalis)

Strongylopsalis laminata Brindle, 1973e: 11, figs 1, 6.

\section{Type material}

1 J , paratype, F3233.1621, dried, mounted on card.

\section{Locality label}

Suri / Bolivia / W. M. Mann. // Mulford / Bio. Exp. / 1921-22.

\section{Taxonomy}

Spongiphoridae, Strongylopsalinae, Strongylopsalis laminata Brindle, 1973.

\section{Remark}

Index card entry: Bolivia, Cajuata.

\section{lamottei Brindle, 1971 (Anisolabis)}

Anisolabis lamottei Brindle, 1971c: 270, figs 5-7, 9.

\section{Type material}

3 ठึ

\section{Locality labels}

F3233.1387-1388: Savane / Sommet 1550 m / 25.V.1963. // Mission ENS-IFAN / Aux Monts Loma / Sierra Leone.

F3233.1389: Savane / 2 metres ou vegetation. Piste de / Bandankoro / 29.V.1963 // Mission ENS-IFAN / Aux Monts Loma / Sierra Leone.

F3233.1390: Savane / 1580 m / 18-V-1963. // Mission ENS-IFAN / Aux Monts Loma / Sierra Leone. F3233.1391: Prairie à / cyperacées / 20.V.1963. // Mission ENS-IFAN / Aux Monts Loma / Sierra Leone.

\section{Taxonomy}

Anisolabididae, Anisolabidinae, Anisolabis lamottei Brindle, 1971.

\section{Remark}

For the specimen most probably corresponding to F3233.1390, the original description gives the altidtude as $1630 \mathrm{~m}$, not $1580 \mathrm{~m}$.

\section{lamottei Hincks, 1954 (Diplatys)}

Diplatys lamottei Hincks, 1954b: 115, figs 17-18.

\section{Type material}

1 , paratype, F3233.1303, dried, mounted on card.

\section{Locality label}

IFAN / Nimba (Guinée) / Lamotte et Roy / VII - XII 51. 


\section{Taxonomy}

Diplatyidae, Diplatyinae, Paradiplatys lamottei (Hincks, 1954).

\section{Remarks}

Transferred to Lobodiplatys lamottei by Steinmann (1974: 199) and to the subgenus Paradiplatys by Steinmann (1989c: 82). The combination Paradiplatys (Paradiplatys) lamottei was reinstated by Engel \& Haas (2007: 19).

\section{lateralis Brindle, 1989 (Carcinophora)}

Carcinophora lateralis Brindle, 1989: 14, fig. 11.

\section{Type material}

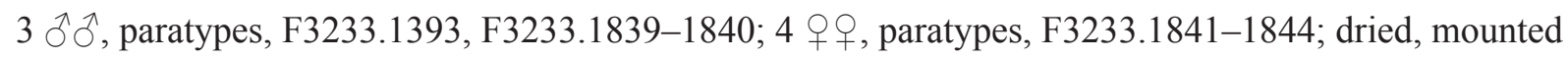
on card.

\section{Locality labels}

F3233.1393: Panama, Fortuna / Wet forest / at light, 1050 m. / 11 Oct. 1976 / Dr. Henk Wolda, STRI. Same data except date: F3233.1840, 1 Oct. 1976; F3233.1843, 7 Oct. 1976; F3233.1844, 15 Oct. 1976; F3233.1841, 24 May 1977; F3233.1839, 6 Sep. 1978; F3233.1842, 24 Sep. 1978.

\section{Taxonomy}

Anisolabididae, Anisolabidinae, Carcinophora lateralis Brindle, 1989.

\section{Remarks}

1. Not listed in Steinmann (1989c) or Hopkins et al. (2014).

2. The date range of the specimens does not match exactly the date range in the original description: 27.iii.1976-2.viii.1977.

\section{leleupi Brindle, 1968 (Anophthalmolabis)}

Anophthalmolabis leleupi Brindle, 1968b: 172, figs 1-2.

\section{Type material}

1 ô, paratype, F3233.101; 1 +, paratype, F3233.100; dried, mounted on card.

\section{Locality labels}

F3233.100-101: Galapagos Isles / Santa Cruz / 27.XII.1964 / N. Leleup.

\section{Taxonomy}

Anisolabididae, Anophthalmolabidinae, Anophthalmolabis leleupi Brindle, 1968.

\section{lenkoi Brindle, 1968 (Brachylabis)}

Brachylabis lenkoi Brindle, 1968a: 32, figs 1-2.

\section{Type material}

$2 \hat{\jmath}$, paratypes, F3233.1424-1425, dried, mounted on card. 


\section{Locality labels}

F3233.1424-1425: Barueri / SP, Brazil / 20.VIII.1967 / K. Lenko col.

\section{Taxonomy}

Anisolabididae, Brachylabidinae, Brachylabis lenkoi Brindle, 1968.

\section{Remark}

Index card entry: Sao Paolo.

\section{lingua Brindle, 1970 (Adiathella)}

Adiathella lingua Brindle, 1970f: 689, fig. 57.

\section{Type material}

1 §ै, paratype, F3233.926, dried, mounted on card.

\section{Locality label}

J. L. Gressit / Collector // Solomon Is. / Guadalcanal : Suta / 500-1200 m. / IV-27-1956.

\section{Taxonomy}

Chelisochidae, Chelisochinae, Adiathella lingua Brindle,1970.

\section{linsleyi Brindle, 1970 (Skalistes)}

Skalistes linsleyi Brindle, 1970b: 226, fig. 4.

\section{Type material}

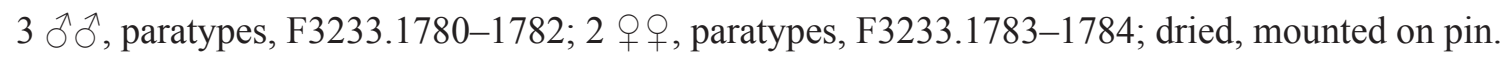

\section{Locality labels}

F3233.1780-1784: Michoacan / Mex. V-13-38 // Pres. by E. G. Linsley

\section{Taxonomy}

Forficulidae, Forficulinae, Skalistes linsleyi Brindle, 1970.

\section{longicollis Hincks, 1957 (Nesolabia)}

Nesolabia longicollis Hincks, 1957c: 54, figs 9-11.

\section{Type material}

1 ô, paratype, F3233.1832; 1 \%, paratype, F3233.1833; dried, mounted on card.

\section{Locality labels}

F3233.1832-1833: La Reunion / Plaine des Cafres / Piton Manuel / 27 I 1955 // Institut / Scientifique / Madagascar.

\section{Taxonomy}

Spongiphoridae, Geracinae, Nesolabia longicollis Hincks, 1957. 


\section{Remark}

The original description makes no reference to a female paratype with data matching that for F3233.1833.

\section{lucasi Brindle, 1974 (Skalistes)}

Skalistes lucasi Brindle, 1974a: 5, figs 1-4.

\section{Type material}

$4 \widehat{\partial}$, paratypes, F3233.1785-1788; 4 Q + , paratypes, F3233.1789-1792; dried, mounted on pin.

\section{Locality labels}

F3233.1785, F3233.1789: Mexico: Chiapas-Oaxaca / border, $21 \mathrm{~km} \mathrm{W.} \mathrm{Rizo} \mathrm{/} \mathrm{de} \mathrm{Oro} \mathrm{along} \mathrm{ridge} \mathrm{SE}$ Cerro Baul, 1615 m. / 8-IX-1972, K. E. Lucas / within Tillandisa sp.

F3233.1791: Same data except 'within Tillandsia guatamalensis / specimen \#: B3-'.

F3233.1786: Same data except 'within Vriesia sp'.

F3233.1792 : Same data except 'within epiphytic Vriesia sp. / specimen \#: B3-'.

F3233.1787-1788, F3233.1790: Same data as F233.1792 except date 8 Jan. 1973.

\section{Taxonomy}

Forficulidae, Forficulinae, Skalistes lucasi Brindle, 1974.

\section{lucens Brindle, 1975 (Forficula)}

Forficula lucens Brindle, 1975c: 48, figs 56-57.

\section{Type material}

1 ô, paratype, F3233.1731; 1 + , paratype, F3233.1732; dried, mounted on card.

\section{Locality labels}

F3233.1731-1732: Tongsa 2150 m / 24/6 // Nat.-Hist. Museum / Basel - Bhutan / Expedition 1972.

\section{Taxonomy}

Forficulidae, Forficulinae, Guanchia lucens (Brindle, 1975).

\section{Remark}

Transferred by Steinmann (1993: 584).

\section{lundbladi Hincks, 1938 (Perirrhytus)}

Perirrhytus lundbladi Hincks, 1938: 5, figs 5-6.

\section{Type material}

3 paratypes, gender unspecified, F3233.1845-1847; dried, mounted on card.

\section{Locality labels}

F3233.1845: Madeira: / Caramujo. / 12.8.35 / "Erica - [illegible]" / Dr. O. Lundblad. / Stockholm Mus. F3233.1846-1847: Madeira: / Feiteiras / 15.8.35 / Dr. O. Lundblad. 


\section{Taxonomy}

Forficulidae, Anechurinae, Perirrhytus lundbladi Hincks, 1938.

\section{Remarks}

Index card entry: $2 \hat{\partial} \hat{\partial}, 1$ + This agrees with the original description, which states that 15 paratypes were examined, that all paratypes with the same locality information as F3233.1845 were males and that the only two described from Feiteiras (as F3233.1846-1847) were $1 \delta$ and $1 q$.

\section{machu-picchuensis Brindle, 1971 (Marava)}

Marava machu-picchuensis Brindle, 1971d: 250, figs 1, 5, 7.

\section{Type material}

1 ऽ, paratype, F3233.1551, dried, mounted on card.

\section{Locality label}

Torentoy Canyon (Ba- / se Machu-Picchu) / 2000-2000m - Peru / VI-VII.964 B. Malkin.

\section{Taxonomy}

Spongiphoridae, Spongiphorinae, Marava machupicchuensis Brindle, 1971.

\section{magnus Brindle, 1988 (Vostox)}

Fig. 41

Vostox magnus Brindle, 1988: 50, figs 9-10.

\section{Type material}

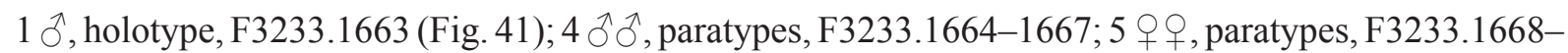
1672; dried, mounted on card.

\section{Type locality}

Panama, 1300M / Alto Lino, near / Boguete, Chiriqui / 25/31.VIII.1976 / Dr. Henk Wolda, STRI.

\section{Locality labels}

F3233.1664: Panama, 1300M / Alto Lino, near / Boguete, Chiriqui / 12-VI-1978 / Dr. Henk Wolda, STRI.

Same data except date: F3233.1665, 6 Sep. 1977; F3233.1668, 3-4 Jun. 1978.

F3233.1666: Panama, Fortuna / Wet forest / at light, 1050m / 23 Nov. 1976 / Dr. Henk Wolda, STRI.

Same data except date: F3233.1667, 29 Nov. 1976; F3233.1671, 12 Apr. 1978; F3233.1670, 15 May 1978; F3233.1672, 14 Aug. 1978; F3233.1669, 25 Aug. 1978.

\section{Taxonomy}

Spongiphoridae, Spongiphorinae, Vostox magnus Brindle, 1988.

\section{malaisei Hincks, 1947 (Chaetospania)}

Chaetospania malaisei Hincks, 1947b: 19, figs 9-11. 


\section{Type material}

1 ऽ, paratype, F3233.1513, dried, mounted on card.

\section{Locality label}

N. E. Burma / Kambaiti 7000 ft / 30/5 1934 / R. Malaise.

\section{Taxonomy}

Spongiphoridae, Sparattinae, Chaetospaniini, Chaetospania malaisei Hincks, 1947.

\section{malgacha Brindle, 1966 (Euborellia)}

Euborellia malgacha Brindle, 1966g: 236, fig. 28.

\section{Type material}

1 ऽ, paratype, F3233.1441, dried, mounted on card.

\section{Locality label}

Sahafanjana / Manambato / (Anove). Institut / Scientifique / Madagascar.

\section{Taxonomy}

Anisolabididae, Anisolabidinae, Euborellia malgacha Brindle, 1966.

\section{malgacha Brindle, 1966 (Labia)}

Labia malgacha Brindle, 1966g: 245, figs 33-35.

\section{Type material}

2 우, paratypes, F3233.1541-1542, dried, mounted on card.

\section{Locality labels}

F3233.1541-1542: Ampitameloka / 840 m / sud Moramanga / VIII/56 P. Griv. // Institut Scientifique / Madagascar.

\section{Taxonomy}

Spongiphoridae, Labiinae, Paralabella malgacha (Brindle, 1966).

\section{Remark}

Transferred by Steinmann (1989b: 486).

\section{mameti Hincks, 1950 (Labia)}

Labia mameti Hincks, 1950b: 354, figs 1-3.

\section{Type material}

1 ऽ, paratype, F3233.1594, dried, mounted on card.

\section{Locality label}

Mauritius / Le Pouce Mt. / 2. XI. 1948 / R. Mamet.26. 


\section{Taxonomy}

Spongiphoridae, Geracinae, Pseudovostox mameti (Hincks, 1950).

\section{Remarks}

1. Transferred by Brindle (1973d: 142).

2. The original description has the collection date as 1949, not 1948 .

\section{martensi Brindle, 1987 (Aborolabis)}

Aborolabis martensi Brindle, 1987: 336, figs 12, 17.

\section{Type material}

1 §, paratype, F3233.1837; 1 \%, paratype, F3233.1838; dried, mounted on card.

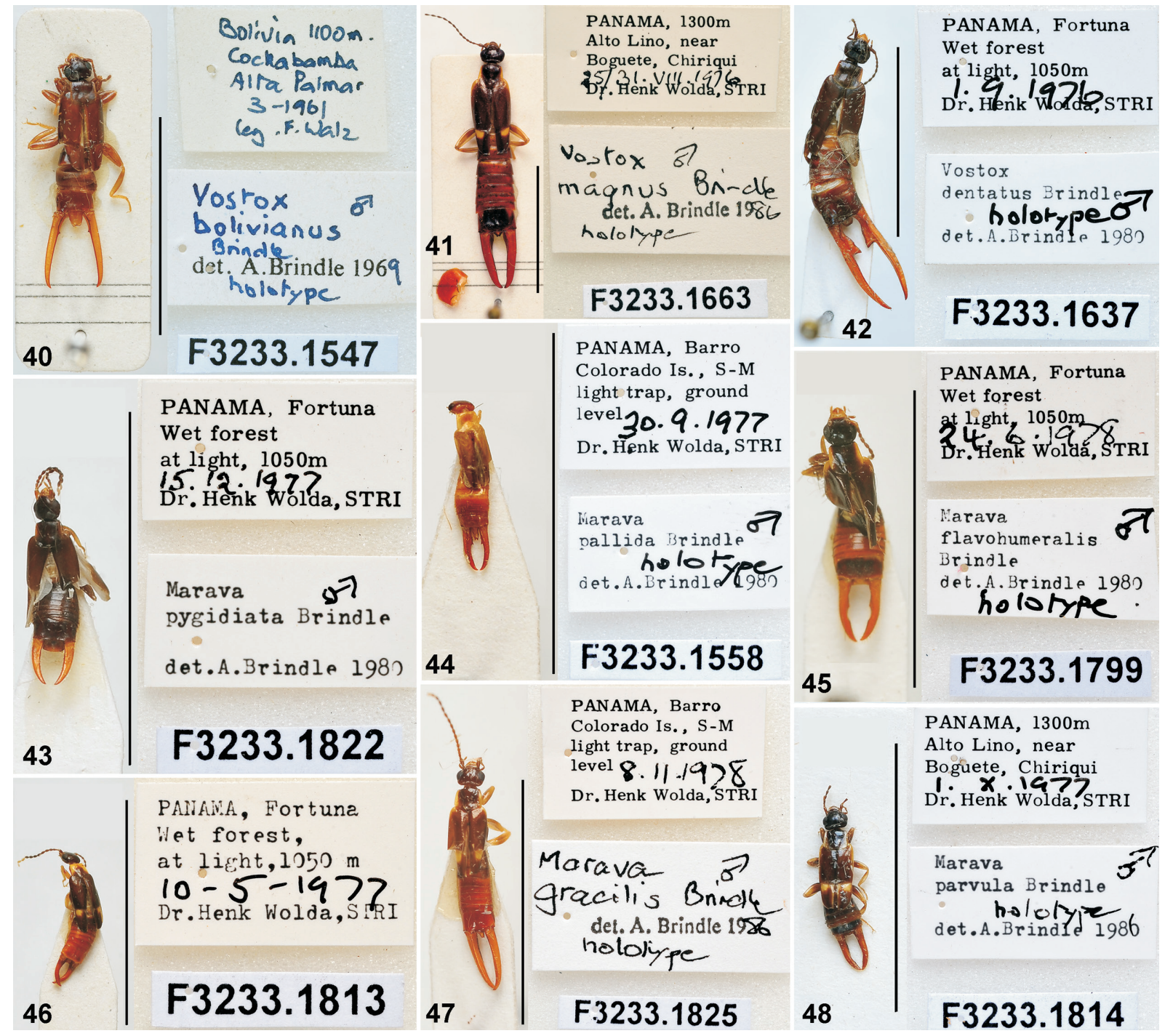

Figs 40-48. Holotypes $\left({ }^{\Uparrow}\right)$ of Spongiphoridae from the collection of the Manchester Museum. 40. Vostox bolivianus Brindle, 1971. 41. V. magnus Brindle, 1988. 42. V. dentatus Brindle, 1988. 43. Marava pygidiata Brindle, 1988. 44. M. pallida Brindle, 1988. 45. M. flavohumeralis Brindle, 1988. 46. M. nigrocincta Brindle, 1988 (no determination label). 47. M. gracilis Brindle, 1988. 48. M. parvula Brindle, 1988. Scale bars $=1 \mathrm{~cm}$. 


\section{Locality labels}

F3233.1837-1838: Nepal, Gorkha / Dist. Buri Gandaki / 29 July 1983 / 700-800 m. 219.

\section{Taxonomy}

Anisolabididae, Anisolabidinae, Aborolabis martensi Brindle, 1987.

\section{Remark}

The original description gives the locality as between Suteo and Labubesi, Shorea.

\section{martensi Brindle, 1974 (Allodahlia)}

Allodahlia martensi Brindle, 1974c: 154, figs 13-16, 18.

\section{Type material}

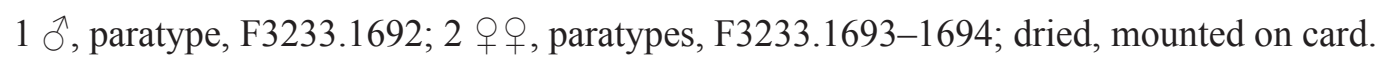

\section{Locality labels}

F3233.1692: Nepal. Thaksang / above Tukche / 3100-3400m. / 6/7.VII.1973 / J. Martens. F3233.1693, same data except: 3150-3200m. / 2/4.VII.1973.

F3233.1694: Nepal 2600m. / Gosainkund / 23.IV.1973 / J. Martens.

\section{Taxonomy}

Forficulidae, Allodahliinae, Allodahlia martensi Brindle, 1974.

\section{Remarks}

The original description states, ' 1 male, 1 female paratypes (32) and 1 male, 1 female paratypes (35) have been retained in the Manchester Museum.' [32 and 35 refer to data sets.] F3233.1692 matches data set 32 in the description except the date 5-10 Aug. 1970. The female with this data appears to be missing from MMUE. F3233.1693 matches data set 34 in the description exactly but not 32 or 35 . The data for F3233.1694 does not match any in the description, except the collector.

\section{masai Hincks, 1949 (Afrolabia)}

Afrolabia masai Hincks, 1949a: 76, figs 1-3.

\section{Type material}

1 ๙ै, paratype, F3233.1609; 1 \&, paratype, F3233.1610; dried, mounted on card.

\section{Locality labels}

F3233.1609-1610: Van Someren / Meru, 7/43.

\section{Taxonomy}

Spongiphoridae, Spongiphorinae, Spongovostox masai (Hincks, 1949).

\section{Remarks}

1. Transferred by Brindle (1973f: 216).

2. Index card entry: Africa, Tanzania, Mt. Meru. 
meridionalis Brindle, 1978 (Brachylabis)

Fig. 22

Brachylabis meridionalis Brindle, 1978: 29, figs 11-13.

Type material

1 ठ , holotype, F3233.1426 (Fig. 22), dried, mounted on card.

\section{Type locality}

S. Africa / Storms R. / 21.9.51 / J F Darbyshire.

\section{Taxonomy}

Anisolabididae, Antisolabidinae, Antisolabis meridionalis (Brindle, 1978).

\section{Remark}

Transferred by Steinmann (1989a: 343).

\section{meridionalis Brindle, 1973 (Chaetospania)}

Fig. 57

Chaetospania meridionalis Brindle, 1973f: 151, fig. 187.

\section{Type material}

1 ô, holotype, F3233.1514 (Fig. 57); 1 क, allotype, F3233.1515; 1 ô paratype, F3233.1516; dried, mounted on card.

\section{Type locality}

Vumba Mountains / S. Rhodesia / Under bark on dead / tree 21.3.1959 / C. N. Smithers.

\section{Locality labels}

F3233.1515-1516: As type locality.

\section{Taxonomy}

Spongiphoridae, Sparattinae, Chaetospaniini, Chaetospania meridionalis Brindle, 1973.

\section{Remark}

The original description refers to two male paratypes, not one.

\section{milloti Hincks, 1953 (Antisolabis)}

Antisolabis milloti Hincks, 1953: 365, figs 4-5.

\section{Type material}

$1 \hat{\jmath}$, paratype, F3233.1484, dried, mounted on card.

\section{Locality label}

17-10-51 / (A.R.) // Institut / Scientifique / Madagascar. 


\section{Taxonomy}

Anisolabididae, Brachylabidinae, Metisolabis milloti (Hincks, 1953).

\section{Remark}

Transferred by Brindle (1966g: 234).

\section{minima Brindle, 1966 (Brachylabis)}

Brachylabis minima Brindle, 1966e: 40, figs 6, 15.

\section{Type material}

1 §, paratype, F3233.1474; 1 \%, paratype, F3233.1475; dried, mounted on card.

\section{Locality labels}

F3233.1474: Forêt transition // Coll. Mus. Congo / Tanganyika Terr.: Uluguru / Mts., Ululu-Ndogo, / 1500 m. 8-V-1957.

F3233.1475: Forêt transition // Coll. Mus. Congo / Tanganyika Terr.: Uluguru / Mts., sommet du Kidunda / $1800-1950$ m. 3-V-1957.

\section{Taxonomy}

Anisolabididae, Antisolabidinae, Antisolabis minima (Brindle, 1966).

\section{Remark}

Transferred by Steinmann (1989a: 348).

\section{minimus Brindle, 1966 (Archidux)}

Archidux minimus Brindle, 1966c: 56, figs 14-16, 20, 23.

\section{Type material}

1 §ै, paratype, F3233.1706; 1 \%, paratype, F3233.1707; dried, mounted on card.

\section{Locality labels}

F3233.1706-1707: Récolté dans / 1'humus // I.R.S.A.C.-Mus. Congo / Kivu : Terr. Lubero / 2.200 m. 27-XI-1951 / N. Leleup / (for. mont. avec bamb.)

\section{Taxonomy}

Forficulidae, Skendylinae, Cosmiella minima (Brindle, 1966).

\section{Remarks}

1. Transferred by Steinmann (1989c: 673).

2. Index card entry: Africa, Zaire.

\section{minuscula Brindle, 1977 (Marava)}

Marava minuscula Brindle, 1977b: 125, figs 23-24.

\section{Type material}

1 ऽ, paratype, F3233.1552; 1 + , paratype, F3233.1553; dried, mounted on card. 


\section{Locality labels}

F3233.1552-1553: Venezuela - Bolivar / El Hormiguero Me- / seta de Nuria. / 500m. 13-17.XII.74.

\section{Taxonomy}

Spongiphoridae, Spongiphorinae, Marava furia Steinmann, 1989.

\section{Remark}

New name by Steinmann (1989c: 502): 'Marava furia Steinmann (new name for Marava minuscula Brindle, 1977 as a junior homonym of Forficula minuscula Latreille, 1817).’

\section{minutissima Brindle, 1972 (Anisolabis)}

Anisolabis minutissima Brindle, 1972c: 128, figs 6a-c, 7b.

\section{Type material}

1 ऽ, paratype, F3233.1394, dried, mounted on card.

\section{Locality label}

Garakayo I. / Pelew Islands / VIII : 9 : 45. // Coll. \& pres. by / Henry S. Dybas.

\section{Taxonomy}

Anisolabididae, Anisolabidinae, Anisolabis minutissima Brindle, 1972.

\section{Remarks}

1. Index card entry: Pelew Islands $=$ Palau.

2. The original description refers only to the holotype and allotype. There is no reference to paratypes.

\section{mucronatus Hincks, 1959 (Esphalmenus)}

Fig. 34

Esphalmenus mucronatus Hincks, 1959: 202, fig. 208.

\section{Type material}

$1 \lesssim$, holotype, F3233.1361 (Fig. 34), dried, mounted on card.

\section{Type locality}

Bolivia / Estacion Condor / 4790 m / 11.I.49. Coll. / Kuschel.

\section{Taxonomy}

Pygidicranidae, Esphalmeninae, Esphalmenus mucronatus Hincks, 1959.

\section{nathani Brindle, 1965 (Gonolabidura)}

Fig. 39

Gonolabidura nathani Brindle, 1965: 594, fig. 21.

\section{Type material}

1 ô, holotype, F3233.1491 (Fig. 39), dried, mounted on card. 


\section{Type locality}

S. India, Coimbatore Dstr. / Valparal 3500 ft. II-III / coll. P. S. Nathan 1938.

\section{Taxonomy}

Labiduridae, Allostethinae, Gonolabidura nathani Brindle, 1965.

\section{nepalensis Brindle, 1974 (Anisolabis)}

Anisolabis nepalensis Brindle, 1974c: 148, figs 4-5.

\section{Type material}

1 §, paratype, F3233.1395; 1 \%, paratype, F3233.1396; dried, mounted on card.

\section{Locality labels}

F3233.1395: Nepal / Myangdi - Khola / Muri 2100 m. / IV.1970 / J. Martens. F3233.1396: Nepal: Sitha - Gorapani / $2300-2700 \mathrm{~m} / \mathrm{J}$. Martens leg. 11.Jul.73.

\section{Taxonomy}

Anisolabididae, Anisolabidinae, Aborolabis nepalensis (Brindle, 1974).

\section{Remarks}

1. Transferred by Steinmann (1979: 215).

2. The original description gives the altitude for F3233.1395 as $2300 \mathrm{~m}$, not $2100 \mathrm{~m}$. The locality of F3233.1396 is given in the description as between Sika (not Sitha as on the label) and Gorapani.

\section{nevermanni Brindle, 1966 (Vara)}

Vara nevermanni Brindle, 1966f: 138, figs 1-4.

\section{Type material}

1 đ̊, paratype, F3233.1749; 2 + 9 , paratypes, F3233.1750-1751; dried, mounted on card.

\section{Locality labels}

F3233.1749: Costa Rica / Farm Hamburg / am Reventazon / 3.XII.1932. // Urwald welkes Laub / F. Nevermann leg. / Eing. Nr.104, 1932.

F3233.1750: Costa Rica / Farm Hamburg / am Reventazon / 29.3.31. // F. Nevermann leg. / Eing. Nr.164, 1931.

F3233.1751: Costa Rica / Turrialba / 29.VIII.32 // An Carica papaya / F. Nevermann leg. / Eing. Nr. 42 1934.

\section{Taxonomy}

Forficulidae, Ancistrogastrinae, Sarcinatrix anomalia (Rehn, 1903).

\section{Remarks}

1. Vara nevermanni Brindle, 1966 synonymised with Sarcinatrix anomalia (Rehn, 1903) by Brindle (1967a: 189).

2. The original description gives the date for the specimen which matches F3233.1751 as 29 Jul. 1932, not 29 Aug. 1932. 
3. In the original description, no reference to a specimen matching the date of F3233.1750 (i.e., 29 Mar. 1931) was made.

\section{nigerrima Brindle, 1966 (Forficula)}

Forficula nigerrima Brindle, 1966b: 148, figs 1-3.

\section{Type material}

1 ऽ, paratype, F3233.1724, dried, mounted on card.

\section{Locality label}

Nyasaland / Mt Mlange / 8.8.1956 / AWR McCrae. // Ridge 7500' / 1⁄2 m ESE of / Little Rus Camp / Little Rus Plat. // Under stones / on peaty / grass mat.

\section{Taxonomy}

Forficulidae, Forficulinae, Afroforficula nigerrima (Brindle, 1966).

\section{Remarks}

1. Transferred by Steinmann (1993: 544).

2. Index card entry: Africa, Malawi.

3. Mt Mlange (locality label) is a variant spelling of Mt. Mlanje or Mt. Mulanje, Malawi.

\section{nigrescens Brindle, 1987 (Aborolabis)}

Aborolabis nigrescens Brindle, 1987: 337, figs 9, 11.

\section{Type material}

1 ऽ, paratype, F3233.1835; 1 \%, paratype, F3233.1836; dried, mounted on card.

\section{Locality labels}

F3233.1835-1836: Nepal, Nuwakot / District, Trisuli / 21/22 July 1983 / 600-650 m 907.

\section{Taxonomy}

Anisolabididae, Anisolabidinae, Aborolabis nigrescens Brindle, 1987.

\section{nigrescens Brindle, 1977 (Pyragra)}

Pyragra nigrescens Brindle, 1977b: 113, figs 6, 8.

\section{Type material}

1 , paratype, F3233.1340, dried, mounted on pin.

\section{Locality label}

Venezuela, Bolivar / El Bochinche Res. / Forestal Imataca / 200m. 6-13.XII.74.

\section{Taxonomy}

Pygidicranidae, Pyragrinae, Pyragra nigrescens Brindle, 1977. 


\section{nigrinus Brindle, 1984 (Esphalmenus)}

Esphalmenus nigrinus Brindle, 1984: 286, fig. 24.

\section{Type material}

1 , paratype, F3233.1362, dried, mounted on card.

\section{Locality label}

Ecuador / N. of Azogues / 3000 m.

\section{Taxonomy}

Pygidicranidae, Esphalmeninae, Esphalmenus nigrinus Brindle, 1984.

\section{Remark}

The original description refers to one male paratype at MMUE. The MMUE specimen is labelled + .

\section{nigrita Brindle, 1971 (Idolopsalis)}

Idolopsalis nigrita Brindle, 1971d: 255, fig. 9.

\section{Type material}

1 đ̊, paratype, F3233.1464, dried, mounted on card. 1 \&, paratype, F3233.1465, dried, mounted on pin.

\section{Locality labels}

F3233.1464: Bromélia / 10m // Est. Biol. Boraceia /Salesópolis, SP / 26.XI.1968 / E. X. Rabello col. F3233.1465: Est. Biol. Boraceia /Salesópolis, SP / 18.VI.1966 / C. Costa col.

\section{Taxonomy}

Anisolabididae, Idolopsalidinae, Idolopsalis nigrita Brindle, 1971.

\section{Remark}

The original description gives the locality San Paulo.

\section{nigritum Brindle, 1974 (Eugerax)}

Eugerax nigritum Brindle, 1974b: 118.

\section{Type material}

1 o, paratype, F3233.1533, dried, mounted on card.

\section{Locality label}

Venezuela - Aragua / Rancho Grande / 1100m. 12.V.73. // J. A. Clavijo / G. Equis.

\section{Taxonomy}

Spongiphoridae, Geracinae, Eugerax nigritum Brindle, 1974. 


\section{nigrocincta Brindle, 1988 (Marava)}

Fig. 46

Marava nigrocincta Brindle, 1988: 52, figs 14, 19.

\section{Type material}

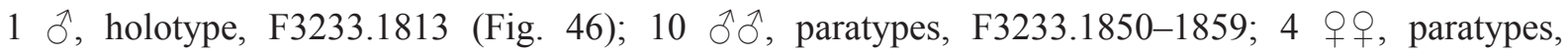
F3233.1860-1863; dried, mounted on card.

\section{Type locality}

Panama, Fortuna / Wet forest / at light, 1050m / 10.5.1977 / Dr. Henk Wolda, STRI.

\section{Locality labels}

As type locality except dates: F3233.1862, 13 Nov. 1976; F3233.1860, 28 Jul. 1977; F3233.1861, 15 Aug. 1977; F3233.1863, 11 Sep. 1977; F3233.1850-1854, 24 Sep. 1977, F3233.1855-1858, 25 Sep. 1977; F3233.1859, 24 Oct. 1977.

\section{Taxonomy}

Spongiphoridae, Spongiphorinae, Marava nigrocincta Brindle, 1988.

\section{Remark}

The original description gives the collection date of the holotype as 21 Jul. 1977, not 10 May 1977 as on the locality label.

\section{nilgiriensis Hincks, 1955 (Diplatys)}

Diplatys nilgiriensis Hincks, 1955a: 111, fig. 144.

\section{Type material}

1 ऽ, paratype, F3233.1304, dried, mounted on card.

\section{Locality label}

S. India / Nilgiri Hills / Cherangode / 3,500' X.50 / P.S. Nathan.

\section{Taxonomy}

Diplatyidae, Diplatyinae, Diplatys (Syndiplatys) nilgiriensis Hincks, 1955.

\section{Remarks}

1. Transferred by Steinmann (1986a: 175).

2. The original description gives the collection date as Nov. 1950, not Oct. 1950 as on the locality label.

\section{nimbaensis Hincks, 1954 (Anisolabis)}

Anisolabis nimbaensis Hincks, 1954b: 103, fig. 1.

\section{Type material}

1 J , paratype, F3233.1397; 1 \%, paratype, F3233.1398; dried, mounted on card. 


\section{Locality labels}

F3233.1397-1398: Mt To (1600 m) / Camp 1. Muséum Paris / Nimba (Guinée) / M. Lamotte II. VI. 42.

\section{Taxonomy}

Anisolabididae, Anisolabidinae, Anisolabis nimbaensis Hincks, 1954.

\section{Remarks}

1. Index card entry: Africa, Guinea, Nimba Mts.

2. The original description refers to 4 paratypes in the Hincks collection.

\section{nodulosa Brindle, 1973 (Labia)}

Labia nodulosa Brindle, 1973c: 395, figs 1-2.

\section{Type material}

1 ऽ̄, paratype, F3233.1543, dried, mounted on card.

\section{Locality label}

Costa Rica / Coronado / 15.II.1931 / F. Nevermann.

\section{Taxonomy}

Spongiphoridae, Labiinae, Paralabella conspicua (Borelli, 1906).

\section{Remark}

Synonymised with L. conspicua (Borelli, 1906) by Brindle (1985: 74). L. conspicua transferred to Paralabella by Steinmann (1989b: 473).

\section{notonoma Hincks, 1952 (Antisolabis)}

Antisolabis notonoma Hincks, 1952a: 214, fig. 1.

\section{Type material}

$1 \widehat{\jmath}$, paratype, F3233.1489, dried, mounted on card.

\section{Locality label}

Mt. Keira / New South Wales / 4 : $12: 1949$ / C. E. Chadwick // Under rotting log.

\section{Taxonomy}

Anisolabididae, Antisolabidinae, Antisolabis notonoma Hincks, 1952.

\section{ocellatus Brindle, 1971 (Vostox)}

Vostox ocellatus Brindle, 1971b: 539, figs 63-65.

\section{Type material}

1 ऽ, paratype, F3233.1635, dried, mounted on card.

\section{Locality label}

Honduras / 3913999 / Primavera / logs. n. y. / Aug. 19, 1939. 


\section{Taxonomy}

Spongiphoridae, Spongiphorinae, Vostox ocellatus Brindle, 1971.

\section{ochracea Brindle, 1966 (Chaetospania)}

Chaetospania ochracea Brindle, 1966g: 241, figs 45-46.

\section{Type material}

1 ऽ, paratype, F3233.1517, dried, mounted on card.

\section{Locality label}

Manambato / (Anove) // Institut Scientifique / Madagascar.

\section{Taxonomy}

Spongiphoridae, Sparattinae, Chaetospaniini, Chaetospania ochracea Brindle, 1966.

\section{ochroptera Brindle, 1972 (Allodahlia)}

Allodahlia ochroptera Brindle, 1972b: 27, fig. 9.

\section{Type material}

1 q, paratype, F3233.1695, dried, mounted on card.

\section{Locality label}

Bhamo / Birmania / Fea IX 1886.

\section{Taxonomy}

Forficulidae, Allodahliinae, Allodahlia ochroptera Brindle, 1972.

\section{Remarks}

1. Bhamo is a city in northern Myanmar.

2. The original description gives the collection date as Oct. 1886, not Sep. 1886 as on the locality label.

\section{pallida Brindle, 1988 (Marava)}

Fig. 44

Marava pallida Brindle, 1988: 51, figs 13, 18.

\section{Type material}

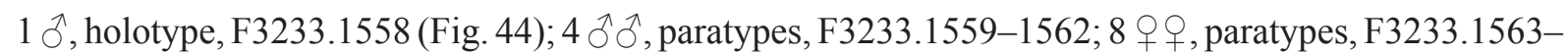
1570 ; dried, mounted on card.

\section{Type locality}

Panama, Barro / Colorado Is., S-M / light trap, ground / level / 30.9.1977 / Dr. Henk Wolda, STRI.

\section{Locality labels}

As type locality except dates: F3233.1562, 22 Nov. 1977; F3233.1560, 1 May 1978; F3233.1559, 17 Jun. 1978; F3233.1561, 8 Sep. 1978; F3233.1563, 27 Sep. 1978; F3233.1568, 13 Oct. 1978; F3233.1567, 
21 Oct. 1978; F3233.1565, 26 Oct. 1978; F3233.1564, 22 Nov. 1978; F3233.1569, 13 Dec. 1978. F3233.1566: Panama, Barro / Colorado Is., S-M / light trap, ground / level / WK 20 D / Dr. Henk Wolda, STRI.

F3233.1570: Panama, Barro / Colorado Is., S-M / light trap, tree / canopy / 30.9.1977 / Dr. Henk Wolda, STRI.

\section{Taxonomy}

Spongiphoridae, Spongiphorinae, Marava pallida Brindle, 1988.

\section{pallidicolle Hincks, 1959 (Echinosoma)}

Echinosoma pallidicolle Hincks, 1959: 154, figs 143-144.

\section{Type material}

1 §, paratype, F3233.461; 2 우, paratypes, F3233.462-463; dried, mounted on card.

\section{Locality labels}

F3233.461: Java / Coll. Gadeau / de Kerville.

F3233.462: Java / Mount Tengger / Coll. Gadeau / de Kerville.

F3233.463: East Java: / Soember Brantas / Feb. 1937. / M. E. Walsh.

\section{Taxonomy}

Pygidicranidae, Echinosomatinae, Echinosoma pallidicolle Hincks, 1959.

\section{pallidus Brindle, 1968 (Auchenomus)}

Auchenomus pallidus Brindle,1968h: 543, figs 4, 7-8, 10.

\section{Type material}

1 ऽ, paratype, F3233.1501, dried, mounted on card.

\section{Locality label}

Virao / S. O. Luzon.

\section{Taxonomy}

Spongiphoridae, Sparattinae, Auchenomini, Auchenomus pallidus Brindle, 1968.

\section{Remark}

Index card entry: Philippine Islands, Luzon.

\section{pandani Hincks, 1960 (Auchenomus)}

Auchenomus pandani Hincks, 1960b: 157, figs 2-3.

\section{Type material}

1 §ै, paratype, F3233.554, dried, mounted on card.

\section{Locality label}

New Guinea / Yalu : Lae / i. 1958 / R. W. Paine. // On leaves of / Pandanus / (No. 4 sp.). 


\section{Taxonomy}

Spongiphoridae, Sparattinae, Auchenomini, Auchenomus pandani Hincks, 1960.

\section{pandani Hincks, 1959 (Tagalina)}

Tagalina pandani Hincks, 1959: 114, fig. 99.

\section{Type material}

1 ऽ, paratype, F3233.58, dried, mounted on pin.

\section{Locality label}

Melambi R. Lae N. G, / Mirilunga Vill. 4500 / 29.12.56 on Pandanus / Coll. J H Ardley (New Guinea) // C. I. F. Coll. / No. 15242.

\section{Taxonomy}

Pygidicranidae, Pygidicraninae, Tagalina pandani Hincks, 1959.

\section{parvula Brindle, 1988 (Marava)}

Fig. 48

Marava parvula Brindle, 1988: 54, fig. 17.

\section{Type material}

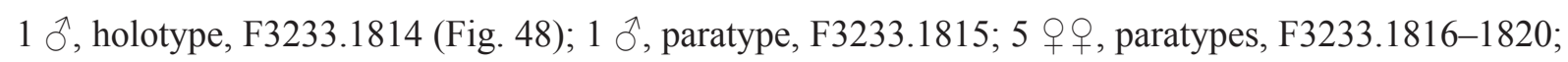

1 paratype, gender unspecified, F3233.1821; dried, mounted on card.

\section{Type locality}

Panama, 1300m / Alto Lino, near / Boguete, Chiriqui / 1.X.1977 / Dr. Henk Wolda, STRI.

\section{Locality labels}

As type locality except dates: F3233.1815, 3 May 1977; F3233.1820, 7 May 1977; F3233.1816, 9 Oct. 1977; F3233.1821, 14 Oct. 1977; F3233.1817, 4 May 1978; F3233.1818, F3233.1819, 23 Jun. 1978.

\section{Taxonomy}

Spongiphoridae, Spongiphorinae, Marava parvula Brindle, 1988.

\section{Remark}

The original description refers to 7 female paratypes at MMUE.

\section{pauliani Hincks, 1948 (Chaetospania)}

Chaetospania pauliani Hincks, 1948: 319, figs 2, 6.

\section{Type material}

1 ऽ, paratype, F3233.1518, dried, mounted on card.

\section{Locality label}

Museum Paris / Cote d'Ivoire / Réserve du Banco / R. Paulian \& C. Delamare. 


\section{Taxonomy}

Spongiphoridae, Sparattinae, Chaetospaniini, Chaetospania pauliani Hincks, 1948.

\section{peruviana Brindle, 1970 (Skalistes)}

Fig. 31

Skalistes peruviana Brindle, 1970b: 223, figs 5, 13.

\section{Type material}

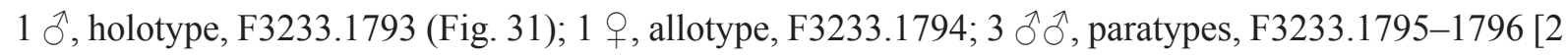
specimens]; dried, mounted on card.

\section{Type locality}

N. Peru : Huambos / nr. Cutervo 2400m / 13.VI.1956. / W. Weyrauch.

\section{Locality labels}

F3233.1794: Porculla-Peru / Pass (Weg von Olmos / nach Jaén) 11. April / leg.: Koepcke. 1953.

F3233.1795-1796: As type locality.

\section{Taxonomy}

Forficulidae, Forficulinae, Skalistes peruviana Brindle, 1970.

\section{philippinense Hincks, 1959 (Echinosoma)}

Echinosoma philippinense Hincks, 1959: 156, figs 148-150.

\section{Type material}

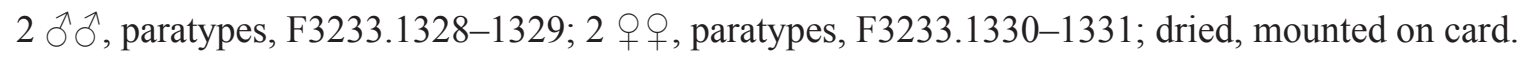

\section{Locality labels}

F3233.1328-1331: Philippine Is. / Mindoro, San / José X.1945 / E. S. Ross. // Ex Calif. / Ac. Scie.

\section{Taxonomy}

Pygidicranidae, Echinosomatinae, Echinosoma philippinense Hincks, 1959.

\section{picea Borelli, 1907 (Gonolabis)}

Gonolabis picea Borelli, 1907c: 1.

\section{Type material}

1 ふָ, syntype, F3233.1399, dried, mounted on card.

\section{Locality label}

1906 Butiti / Viag. Ruwenzori / S. A. R. D. Abruzzi. ex coll. / Borelli.

\section{Taxonomy}

Anisolabididae, Anisolabidinae, Anisolabis duplicata Steinmann, 1989; 


\section{Remarks}

1. Steinmann (1989a: 94) proposed a new name for Gonolabis picea Borelli, 1907, a junior homonym of Gonolabis picea Shiraki, 1905.

2. Index card entry: Uganda.

\section{pieli Hincks, 1940 (Timomenus)}

Fig. 29

Timomenus pieli Hincks, 1940: 38.

\section{Type material}

$1 \hat{\jmath}$, holotype, F3233.1797 (Fig. 29); 1 ô, paratype, F3233.1798; dried, mounted on pin.

\section{Type locality}

Hainan / Nodoa 25.III.36 / G. Ros leg. // 43.

\section{Locality label}

F3233.1798: Hainan Is. S. China / Tsi-pin-tsuen Lam- / ks-heung, Lai-mo-ling / (Mt. Range), Ktungshan / District, May 17-26/1935. F. K. To.

\section{Taxonomy}

Forficulidae, Opisthocosmiinae, Timomenus pieli Hincks, 1940.

\section{ponapensis Brindle, 1972 (Chaetospania)}

Chaetospania ponapensis Brindle, 1972c: 149, fig. 13g-i.

\section{Type material}

$1 \hat{\partial}$, paratype, F3233.607; 2 + , , paratypes, F3233.608-609; dried, mounted on card.

\section{Locality labels}

F3233.607: Nanpil, Nett Dist. / Ponape I. / 25 Feb. 1948.

F3233.609: Same data except date 28 Feb. 1948.

F3233.608: Mt. Tama- / tamansakir / Ponape Id. / 1400' / June - Sept. 50 / P.A. Adams.

\section{Taxonomy}

Spongiphoridae, Sparattinae, Chaetospaniini, Chaetospania ponapensis Brindle, 1972.

\section{Remark}

Index card entry: Micronesia, Ponape.

\section{pophami Brindle, 1969 (Vandex)}

Fig. 51

Vandex pophami Brindle, 1969d: 190, fig. 1.

\section{Type material}

1 Oे, holotype, F3233.1629 (Fig. 51), dried, mounted on card. 
Type locality

Mufindi / Tanganyika / 1938.

\section{Taxonomy}

Spongiphoridae, Vandicinae, Vandex pophami Brindle, 1969.

\section{porrectella Brindle, 1978 (Anisolabis)}

Anisolabis porrectella Brindle, 1978: 86, figs 99-101, 116, 238.

\section{Type material}

1 §, paratype, F3233.1400; 1 \%, paratype, F3233.1401; dried, mounted on card.

\section{Locality labels}

F3233.1400-1401: Récolté dans l'humus. I.R.S.A.C. - Mus. Congo / Kivu: T. Kalehe, Bitale / N. O. Kahusi, 1600 m. / (for. transit.) 29-VI-1951 / N. Leleup.

\section{Taxonomy}

Anisolabididae, Anisolabidinae, Anisolabis porrectella Brindle, 1978.

\section{Remark}

The original description gives the locality as Kahele Territory, not Kalehe as on the locality label. Bitale is in Kalehe Territory, South Kivu Province, Democratic Republic of Congo.

\section{prolongata Hincks, 1947 (Sphingolabis)}

Sphingolabis prolongata Hincks, 1947b: 21, figs 12-13.

\section{Type material}

1 ऽ, paratype, F3233.1599, dried, mounted on card.

\section{Locality label}

N. E. Burma / Kambaiti 7,000 ft. / 12/4 R. Malaise.

\section{Taxonomy}

Spongiphoridae, Labiinae, Sphingolabis prolongata Hincks, 1947.

\section{Remarks}

1. Hopkins et al. (2014) has 'prolonga'.

2. The original description gives the full date as 12 Apr. 1934 .

\section{pulchellus Brindle, 1977 (Purex)}

Purex pulchellus Brindle, 1977b: 118, figs 9-11.

\section{Type material}

1 đ, paratype, F3233.1596; 1 \%, paratype, F3233.1597; dried, mounted on card. 


\section{Locality labels}

F3233.1596: Venezuela - Aragua / Rancho Grande / 1100m. 20.V.75. // En la / Luz. // J. Salcedo / J. A. Clavijo.

F3233.1597: Same data except date 2 Apr. 1975 and collector J. Salcedo.

\section{Taxonomy}

Spongiphoridae, Spongiphorinae, Purex pulchellus Brindle, 1977.

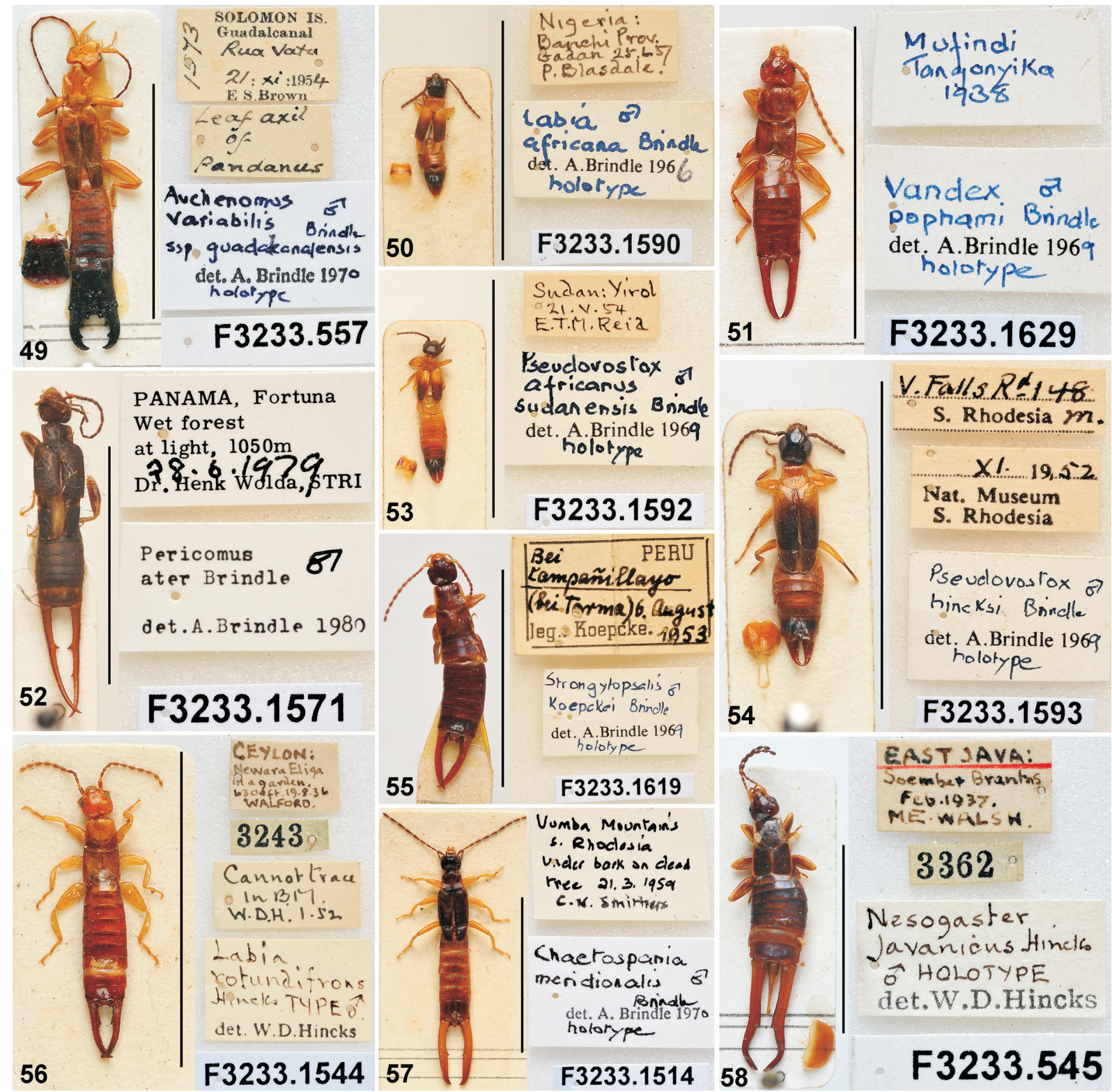

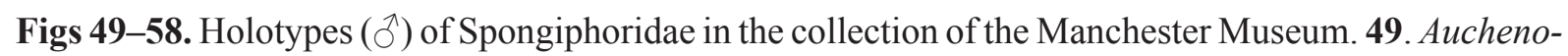
mus variabilis guadalcanalensis Brindle, 1970. 50. Labia africana Brindle, 1968 (now Pseudovostox africanus). 51. Vandex pophami Brindle, 1969. 52. Pericomus ater Brindle, 1988. 53. Pseudovostox africanus sudanensis Brindle, 1970. 54. P. hincksi Brindle, 1970. 55. Strongylopsalis koepckei Brindle, 1968. 56. Labia rotundifrons Hincks, 1954 (now Paralabella rotundifrons). 57. Chaetospania meridionalis Brindle, 1973. 58. Nesogaster javanicus Hincks, 1951 (now N. aculeatus). Scale bars $=1$ $\mathrm{cm}$. 


\section{pusilla Brindle, 1978 (Bormansia)}

Bormansia pusilla Brindle, 1978: 189, fig. 347.

\section{Type material}

1 ô, paratype, F3233.1337, dried, mounted on card.

\section{Locality label}

Ostafrika: T. T. / Msala (Rufiji) / 27.IV.1952 / H. Knipper leg.

\section{Taxonomy}

Karschiellidae, Bormansia pusilla Brindle, 1978.

\section{Remark}

Index card entry: Tanzania.

\section{pygidiata Brindle, 1988 (Marava)}

Fig. 43

Marava pygidiata Brindle, 1988: 54, fig. 23.

\section{Type material}

1 O, holotype, F3233.1822 (Fig. 43); 2 우, paratypes, F3233.1823-1824; dried, mounted on card. In spirit: 2 paratypes [gender not specified], F3448.31.

\section{Type locality}

Panama, Fortuna / Wet forest / at light, 1050m / 15.12.1977 / Dr. Henk Wolda, STRI.

\section{Locality labels}

As type locality except date: F3233.1823, 24 Sep. 1977; F3233.1824, 25 Sep. 1977; F3448.31, 19771978.

\section{Taxonomy}

Spongiphoridae, Spongiphorinae, Marava pygidiata Brindle, 1988.

\section{Remark}

The original description (Brindle 1988) refers to one male and 12 female paratypes at MMUE with a date range of 12 Aug. 1977-3 Jun. 1979.

\section{pygidiatus Brindle, 1975 (Vandex)}

Vandex pygidiatus Brindle, 1975b: 687, figs 5-7.

\section{Type material}

1 O , paratype, F3233.1630; 1 \&, paratype, F3233.1631; dried, mounted on card.

\section{Locality labels}

F3233.1630: Tanzanie : Mts Uluguru / Morning Side, Toelo / for., alt. 1450 m / 21-29/VI/71.

F3233.1631: Tanzanie : Mts Uluguru / Chenzema, alt. 1700m. / 2-22/VII/71. 


\section{Taxonomy}

Spongiphoridae, Vandicinae, Vandex pygidiatus Brindle, 1975.

\section{pygmaea Brindle, 1970 (Metresura)}

Metresura pygmaea Brindle, 1970a: 105, fig. 9.

\section{Type material}

1 ठ

\section{Locality labels}

F3233.1741: La Venta - D.F. / Mexico / 17.XII.1961 / PE. Pereira Col.

F3233.1742: Desierto Los Leones / 2900 m. D.F. Mexico / II - 1962 / Olguin E Pereira.

\section{Taxonomy}

Forficulidae, Neolobophorinae, Metresura pygmaea Brindle, 1970.

\section{quadricollis Hincks, 1954 (Apolabis)}

Apolabis quadricollis Hincks, 1954b: 106, fig. 6.

\section{Type material}

1 o, paratype, F3233.1402, dried, mounted on card.

\section{Locality label}

Muséum Paris / Nimba (Guinée) / II. VI. 42. Pierre Richaud (900m).

\section{Taxonomy}

Anisolabididae, Anisolabidinae, Anisolabis quadricollis (Hincks, 1954).

\section{Remarks}

1. Transferred by Brindle (1978: 114).

2. Index card entry: Guinea, Nimba Mts.

\section{quadrimaculata Brindle, 1971 (Sarcinatrix)}

Sarcinatrix quadrimaculata Brindle, 1971g: 169, figs 12-16.

\section{Type material}

1 o, paratype, F3233.1746, dried, mounted on card.

\section{Locality label}

Est. Biol. Boraceia / Salesópolis - S. P. / Brasil - 13 - 18.IV.961 / Reichardt col.

\section{Taxonomy}

Forficulidae, Ancistrogastrinae, Sarcinatrix quadrimaculata Brindle, 1971.

\section{Remark}

Index card entry: Brazil, Sao Paulo, Estação Biologica de Boracéia, Salesópolis. 


\section{rehni Hincks, 1951 (Nesogaster)}

Nesogaster rehni Hincks, 1951b: 573, fig. 17.

\section{Type material}

$2 \widehat{\partial}$, paratypes, F3233.537, dried, pinned, double mounted on the same pin.

\section{Locality label}

Queensland / Kuranda / Dodd.

\section{Taxonomy}

Spongiphoridae, Nesogastrinae, Nesogaster rehni Hincks, 1951.

\section{reichardti Brindle, 1971 (Mecomera)}

Mecomera reichardti Brindle, 1971g: 166, figs 1-7.

\section{Type material}

1 Oे, paratype, F3233.1520; 1 q, paratype, F3233.1521; dried, mounted on card.

\section{Locality labels}

F3233.1520-1521: St. Catharina / Blumenau / [illegible] / 20.339 / II.19.

\section{Taxonomy}

Spongiphoridae, Sparattinae, Sparattini, Mecomera reichardti Brindle, 1971.

\section{Remark}

Index card entry: Blumenau, Santa Catarina, Brazil.

\section{rhodesiensis Brindle, 1973 (Afrocosmia)}

Afrocosmia rhodesiensis Brindle, 1973f: 288, figs 389-391.

\section{Type material}

1 đે, paratype, F3233.1679; 1 q, paratype, F3233.1680; dried, mounted on card.

\section{Locality labels}

F3233.1679: Récolté dans / 1'humus // I.R.S.A.C.-Mus. R.A.C. / S. Rhodesia : Mt Selinda, / forêt de Chirinda, 1300 m. / VII. 1960 N. Leleup.

F3233.1680: Vase d'une / tête de source / marécageuse // I.R.S.A.C.-Mus. R.A.C. / S. Rhodesia : Mt Selinda, / forêt de Chirinda, 1300 m. / VII. 1960 N. Leleup.

\section{Taxonomy}

Forficulidae, Skendylinae, Afrocosmia rhodesiensis Brindle, 1973.

$$
\text { rileyi Hincks, } 1955 \text { (Diplatys) }
$$

Diplatys rileyi Hincks, 1955a: 74, figs 75-78. 
Type material

1 , paratype, F3233.1305, dried, mounted on card.

\section{Locality label}

Sikkim: Singhik / 3,500 ft. 23.iv.1924. / Maj. R. W. G. Hingston.

\section{Taxonomy}

Diplatyidae, Diplatyinae, Haplodiplatys rileyi (Hincks, 1955).

\section{Remark}

Transferred by Steinmann (1974: 203).

rostrata Brindle, 1978 (Anisolabis)

Anisolabis rostrata Brindle, 1978: 87, figs 107-109, 237.

\section{Type material}

1 ऽ, paratype, F3233.1403, dried, mounted on card.

\section{Locality label}

Biot. No. 95 / Humus / en forêt. Mus. Roy. Afr. Centr. / Tshuapa : Terr. Ikela, / riv. Lukendu, VIII-1959 / B. 95 N. Leleup.

\section{Taxonomy}

Anisolabididae, Anisolabidinae, Anisolabis rostrata Brindle, 1978.

\section{rostratus Brindle, 1984 (Esphalmenus)}

Esphalmenus rostratus Brindle, 1984: 288, fig. 27.

\section{Type material}

1 ภ, paratype, F3233.1363; 1 \&, paratype, F3233.1364; dried, mounted on card.

\section{Locality labels}

F3233.1363-1364: Argentina / Tierra del Fuego / Estancia Viamonte / Auricosta 23.I.1979 / Mision Cientifica Danesa.

\section{Taxonomy}

Pygidicranidae, Esphalmeninae, Esphalmenus rostratus Brindle, 1984.

\section{rotundicollis Hincks, 1952 (Diplatys)}

Diplatys rotundicollis Hincks, 1952b: 19, figs 1-6.

\section{Type material}

2 Љึ, paratypes, F3233.1307, F3233.1849; 2 우, paratypes, F3233.1308-1309; dried, mounted on card. 


\section{Locality labels}

F3233.1307: Sierra Leone / Njala // 7.III.32. / Hargreaves.

F3233.1308: Njala / Sierra Leone / E. Hargreaves. / Date 24-V-26.

As F3233.1307 except dates: F3233.1309, 2 Mar. 1932; F3233.1849, 11 May 1932.

\section{Taxonomy}

Diplatyidae, Diplatyinae, Paradiplatys rotundicollis (Hincks, 1952).

\section{Remarks}

Transferred to Lobodiplatys (Heterodiplatys) by Steinmann (1989c: 79). The combination Paradiplatys (Heterodiplatys) (Hincks) was reinstated by Engel \& Haas (2007: 19).

\section{rotundifrons Hincks, 1954 (Labia)}

Fig. 56

Labia rotundifrons Hincks, 1954e: 20, figs 14-15.

\section{Type material}

1 ภे, holotype, F3233.1544 (Fig. 56), dried, mounted on card.

\section{Type locality}

Ceylon: / Newara Eliya / in a garden / $6300 \mathrm{ft}$ 19.8.36 / Walford.

\section{Taxonomy}

Spongiphoridae, Labiinae, Paralabella rotundifrons (Hincks, 1954).

\section{Remarks}

1. Transferred by Steinmann (1989b: 496).

2. The original description gives the locality as Nuwara. Newara Eliya (locality label) is a city in Central Province, Sri Lanka.

\section{rougemonti Brindle, 1978 (Anisolabis)}

Fig. 26

Anisolabis rougemonti Brindle, 1978: 135, figs 225-227, 270.

\section{Type material}

1 Oे, holotype, F3233.1404 (Fig. 26); 1 क , allotype, F3233.1405; dried, mounted on card.

\section{Type locality}

Ethiopia / Arossi Province / Sodere, VII-1971 / G. de Rougemont.

\section{Locality label}

F3233.1405: As type locality.

\section{Taxonomy}

Anisolabididae, Anisolabidinae, Anisolabis rougemonti Brindle, 1978. 


\section{Remark}

The original description gives the locality as Sodare, not Sodere as on the specimen label. Arussi Province corresponds to the present day Arsi Zone of Ethiopia.

\section{rouxioides Brindle, 1976 (Brachylabis)}

Brachylabis rouxioides Brindle, 1976b: 74, figs 9, 15, 17.

\section{Type material}

1 Oे, paratype, F3233.119, dried, mounted on pin.

\section{Locality label}

New Caledonia. / P.D. Montague. / 1918-87. Loc. Mt Mou 477 / Date 10.3.14 Sex / Coll. P.D. Montague. / New Caledonia Exped.

\section{Taxonomy}

Anisolabididae, Antisolabidinae, Antisolabis rouxioides (Brindle, 1976).

\section{Remarks}

Transferred by Steinmann (1989a: 361) as Antisolabis rouxiodes. There are inconsistencies in the spelling of rouxiodes/rouxioides in both Steinmann 1989a and Steinmann 1989c, each publication containing both spellings. Hopkins et al. (2014) has 'rouxiodes' and gives 'rouxioides' as a synonym, but with a query. Brindle's original spelling was 'rouxioides'.

\section{royi Brindle, 1971 (Anisolabis)}

Anisolabis royi Brindle, 1971c: 268, figs 1-4, 8.

\section{Type material}

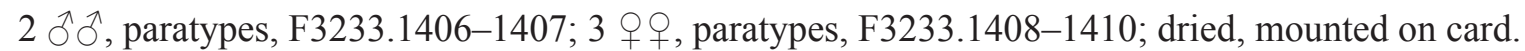

\section{Locality labels}

F3233.1406-1410: Forêt Camp / 1070 m / 26. V. 1963. Mission ENS-IFAN / aux Monts Loma / Sierra Leone.

\section{Taxonomy}

Anisolabididae, Anisolabidinae, Anisolabis royi Brindle, 1971.

\section{Remark}

The original description refers to 12 paratypes at MMUE.

\section{rubescens Brindle, 1973 (Spongovostox)}

Spongovostox rubescens Brindle, 1973f: 222, figs 296-297, 300, 306.

\section{Type material}

1 +, paratype, F3233.1611, dried, mounted on card. 


\section{Locality label}

Bitale Kivu 1954 / R. Pierlot / RP. 48a. // Coll. R. Mayne / Com. Et. Bois Congo R. 2558.

\section{Taxonomy}

Spongiphoridae, Spongiphorinae, Spongovostox rubescens Brindle, 1973.

\section{rudebecki Brindle, 1969 (Pseudovostox)}

Pseudovostox rudebecki Brindle, 1969c: 147, figs 1-5, 9.

\section{Type material}

$1 \hat{\jmath}$, paratype, F3233.1595, dried, mounted on card.

\section{Locality label}

At light in / the evening // Afr. Angola / Vila Artur de Paiva / 5-8.X.1956 / coll. G. Rudebeck.

\section{Taxonomy}

Spongiphoridae, Geracinae, Pseudovostox rudebecki Brindle, 1969.

\section{ruficollis Hincks, 1957 (Brachylabis)}

Brachylabis ruficollis Hincks, 1957b: 69, figs 29-30.

\section{Type material}

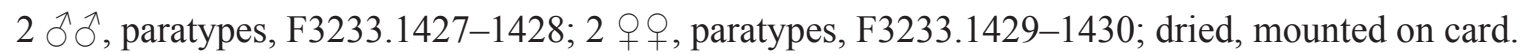

\section{Locality labels}

F3233.1427: Salisbury S. A. / JAN. 1954 / R. M. Arnold.

F3233.1428: South Africa / Grahamstown / 28.9 49 / (R. Bigalke).

F3233.1429: Grahamstown / July 1951 / coll: V. B Brigg.

F3233.1430: Cape Province / Grahamstown / 8.1951 / C D Quickelbirg.

\section{Taxonomy}

Anisolabididae, Brachylabidinae, Ctenisolabis ruficollis (Hincks, 1957).

\section{Remark}

Transferred by Steinmann (1989a: 292).

\section{rugosa Brindle, 1966 (Brachylabis)}

Brachylabis rugosa Brindle, 1966e: 38, fig. 12.

\section{Type material}

1 ô, paratype, F3233.1476; 1 q, paratype, F3233.1477; dried, mounted on card.

\section{Locality labels}

F3233.1476-1477: Vest. Forêt / ombrophile / dans l'humus // Coll. Mus. Congo / Tanganyika Terr.; Bundu- / ki, Uluguru Mts., 1300 m. / 2-V-1957 // Mission Zoolog. I.R.S.A.C. / en afrique orientale / (P. Basilewsky et / N. Leleup). 


\section{Taxonomy}

Anisolabididae, Antisolabidinae, Antisolabis rugosa (Brindle, 1966).

\section{Remark}

Transferred by Steinmann (1989a: 347).

\section{rugosa Brindle, 1975 (Metresura)}

Fig. 28

Metresura rugosa Brindle, 1975d: 84.

\section{Type material}

1 ठे, holotype, F3233.1743 (Fig. 28); 1 क, allotype, F3233.1744; dried, mounted on card.

\section{Type locality}

Salvador, Isalco / volcano, forest / 1200 m., foot of / Cerro Verde (Mala / Cara) Hac. Buena / Vista 26.7.1951.

\section{Locality label}

F3233.1744: As type locality.

\section{Taxonomy}

Forficulidae, Neolobophorinae, Metresura rugosa Brindle, 1975.

\section{rugosula Hincks, 1957 (Forficula)}

Forficula rugosula Hincks, 1957b: 89, fig. 37.

\section{Type material}

1 ô, paratype, F3233.1725, dried, mounted on card.

\section{Locality label}

R. Howell / Grahamstown / Aug 52.

\section{Taxonomy}

Forficulidae, Forficulinae, Guanchia rugosula (Hincks, 1957).

\section{Remark}

Transferred by Steinmann (1993: 557).

\section{rugosus Brindle, 1971 (Anisolabis)}

Anisolabis rugosus Brindle, 1971c: 274, figs 16-19.

\section{Type material}

1 ô, paratype, F3233.1411, dried, mounted on card.

\section{Locality label}

Camp à 800 m / forêt primaire / 17. V. 1963. Mission ENS-IFAN / aux Monts Loma / Sierra Leone. 


\section{Taxonomy}

Anisolabididae, Anisolabidinae, Anisolabis rugosa Brindle, 1971.

\section{Remark}

Brindle (1971c: 266) refers to two paratypes at MMUE.

rugosus Brindle, 1975 (Archidux)

Archidux rugosus Brindle, 1975b: 693.

\section{Type material}

1 §, paratype, F3233.1708; 1 \%, paratype, F3233.1709; dried, mounted on card.

\section{Locality labels}

F3233.1708-1709: Tanzanie : Mts Uluguru / Kinola, for. transition / alt. 1500-1750 m. / 6-13/VI/71 // Coll. Mus. Tervuren / Mission Mts Uluguru / L. Berger, N. Leleup / J. Debecker V/VIII/71.

\section{Taxonomy}

Forficulidae, Skendylinae, Cosmiella rugosa (Brindle, 1975).

\section{Remark}

Transferred by Steinmann (1989c: 674).

\section{saegeri Brindle, 1968 (Nala)}

Nala saegeri Brindle, 1968e: 21, figs 11-12.

\section{Type material}

2 ઊో , paratypes, F3233.1495, F3233.1497; 2 우우, paratypes, F3233.1496, F3233.1498; dried, mounted on card.

\section{Locality labels}

F3233.1495, F3233.1497: Congo belge, P. N. G. / Miss. H. De Saeger / K. 17, 18-iv-1950 / Réc. H. De Saeger. 432.

F3233.1496: Ssame data except date and number, 10 May 1950 and 504.

F3233.1498: Same data as F3233.1496 except number, 506.

\section{Taxonomy}

Labiduridae, Nalinae, Nala saegeri Brindle, 1968.

\section{salcedoi Brindle, 1974 (Eugerax)}

Eugerax salcedoi Brindle, 1974b: 117, figs 9, 13.

\section{Type material}

1 §, paratype, F3233.1534; 1 o, paratype, F3233.1535; dried, mounted on card. 


\section{Locality labels}

F3233.1534:Venezuela - Aragua / Rancho Grande / 1100m. 15-22.VII.74 // Col. Jon H. Soeder.

F3233.1535: Same data except date and collectors: 19.VII.73 // J. Salcedo / J. A. Clavijo.

\section{Taxonomy}

Spongiphoridae, Geracinae, Eugerax salcedoi Brindle, 1974.

\section{salvadorensis Brindle, 1971 (Spongiphora)}

Spongiphora salvadorensis Brindle, 1971b: 531, figs 15, 19.

\section{Type material}

1 J, paratype, F3233.1600; 1 \%, paratype, F3233.1601; dried, mounted on card.

\section{Locality labels}

F3233.1600-1601: El Salvador: Mt. / San Salvador / 4000' - 6400' / VII-8-1963. // D. Q. Cavagnaro / \& M. E. Irwin / collectors.

\section{Taxonomy}

Spongiphoridae, Spongiphorinae, Spongiphora salvadorensis Brindle, 1971.

\section{Remark}

The original description gives the collection date as 1968 (possible misreading of 1963).

\section{salvazae Burr, 1917 (Diplatys)}

Diplatys salvazae Burr, 1917: 57.

\section{Type material}

1 ô, paratype, F3233.1299; 1 \%, paratype, F3233.1300; dried, mounted on card.

\section{Locality labels}

F3233.1299-1300: Cambodia / Pnom Penh / iv 13 Vitalis. // Burr Coll. B. M. 1915 - 120.

\section{Taxonomy}

Diplatyidae, Diplatyinae, Paradiplatys salvazae (Burr, 1917).

\section{Remarks}

Transferred to Lobodiplatys (Paradiplatys) salvazae (Burr, 1917) by Steinmann (1986a: 178). The combination Paradiplatys (Paradiplatys) salvazae (Burr) was reinstated by Engel \& Haas (2007: 20).

\section{schwarzi var. nigrinus Brindle, 1988 (Spongovostox)}

Spongovostox schwarzi var. nigrinus Brindle, 1988: 46, 51, fig. 12.

\section{Type material}

1 paratype [gender unspecified], F3233.1866; dried, mounted on card. 


\section{Locality label}

Panama, Barro / Colorado Is., S-M / light trap, ground / level 5.5.1978 / Dr Henk Wolda, STRI.

\section{Taxonomy}

Spongiphoridae, Spongiphorinae, Spongovostox schwarzi (Caudell, 1907) var. nigrinus Brindle, 1988.

\section{scotti Brindle, 1978 (Anisolabis)}

Anisolabis scotti Brindle, 1978: 131, figs 195-196, 260.

\section{Type material}

1 ऽ, paratype, F3233.1412, dried, mounted on card, without head.

\section{Locality label}

By sifting humus under trees. Abyssinia: / Mt. Chillàlo, / forest, / circa 9,000ft. / 13.xi.1926. / Dr. H. Scott.

\section{Taxonomy}

Anisolabididae, Anisolabidinae, Anisolabis scotti Brindle, 1978.

\section{setulosum Hincks, 1959 (Echinosoma)}

Echinosoma setulosum Hincks, 1959: 155, figs 145-147.

\section{Type material}

1 ऽ, paratype, F3233.1332; 1 \&, paratype, F3233.1333; dried, mounted on card.

\section{Locality labels}

F3233.1332: Tonkin / Than-Moi / Juni-Juli / H. Fruhstorfer / Ex Coll. Gadeau / de Kerville.

F3233.1333: Annam / Phuc-Son / Nov. Dez. / H. Fruhstorfer / Coll. Gadeau / de Kerville.

\section{Taxonomy}

Pygidicranidae, Echinosomatinae, Echinosoma setulosum Hincks, 1959.

\section{seychellensis Brindle, 1976 (Brachylabis)}

Brachylabis seychellensis Brindle, 1976a: 439, figs 1-3.

\section{Type material}

1 §, paratype, F3233.1431; 1 + , paratype, F3233.1432; dried, mounted on card.

\section{Locality labels}

F3233.1431-1432: Iles Séychelles - Mahé / Centre: Morne Blanc / forêt endémique, / 667 m 8/25. VIII.1972.

\section{Taxonomy}

Anisolabididae, Antisolabidinae, Antisolabis seychellensis (Brindle, 1976). 


\section{Remark}

Transferred by Steinmann (1989a: 350).

\section{simiensis Brindle, 1978 (Anisolabis)}

Anisolabis simiensis Brindle, 1978: 134, figs 231-234.

\section{Type material}

1 ô, paratype, F3233.1413, dried, mounted on card.

\section{Locality label}

Ethiopia. c. 3000m, / Simien Mountains / between Debark and / Adesghie. 22.X.1973 / G. de Rougemont.

\section{Taxonomy}

Anisolabididae, Anisolabidinae, Anisolabis simiensis Brindle, 1978.

\section{Remark}

The original description gives the locality as 'near Derasghie'.

\section{simplex Brindle, 1966 (Archidux)}

Archidux simplex Brindle, 1966e: 47, figs 23-25.

\section{Type material}

$2 \AA \widehat{\partial}$, paratypes, F3233.1710-1711, dried, mounted on card.

\section{Locality labels}

F3233.1710-1711: Forêt / transition, / dans l'humus // Coll. Mus. Congo / Tanganyika Terr. : Bundu- / ki, Uluguru Mts., 1500 m. / gorge Mungula 1/6-V-1957. // Mission Zoolog. I.R.S.A.C. / en Afrique orientale / (P. Basilewsky et / N. Leleup).

\section{Taxonomy}

Forficulidae, Skendylinae, Paracosmiella simplex (Brindle, 1966).

\section{Remark}

Transferred by Steinmann (1993: 166).

sogai Brindle, 1966 (Emboros)

Emboros sogai Brindle, 1966g: 252, figs 58, 64.

\section{Type material}

1 +, paratype, F3233.1687, dried, mounted on card.

\section{Locality label}

Madagascar-Est / dct. Andapa / Anjanaharibe 1600m / IV-60 P. Soga. 


\section{Taxonomy}

Forficulidae, Skendylinae, Brachycosmiella sogai (Brindle, 1966).

\section{Remark}

Transferred by Steinmann (1993: 165).

\section{solomonensis Brindle, 1970 (Epilandex)}

Epilandex solomonensis Brindle, 1970f: 654, figs 9-13.

\section{Type material}

5 ठึત, paratypes, F3233.127-131; 4 우, paratypes, F3233.132-135; dried, mounted on card.

\section{Locality labels}

F3233.127-128, F3233.130-135: Solomon Is. Guadacanal [sic] / Mt. Austen / Jan. 1966 / P. Greenslade. F3233.129: Same data except date Sep.-Oct. 1965.

\section{Taxonomy}

Anisolabididae, Anisolabididae, Epilandex solomonensis Brindle, 1970.

\section{spicata Brindle, 1972 (Chaetolabia)}

Chaetolabia spicata Brindle, 1972c: 153, fig. 14g-h.

\section{Type material}

1 Oे, paratype, F3233.618, dried, mounted on card.

\section{Locality label}

Palau: Peleliu / N. E. Coast / 28 Jan 1948 / H.S. Dybas.

\section{Taxonomy}

Spongiphoridae, Labiinae, Chaetolabia spicata Brindle, 1972.

\section{spiculifera Brindle, 1977 (Parasparatta)}

Parasparatta spiculifera Brindle, 1977b: 116, fig. 20.

\section{Type material}

1 O , paratype, F3233.1524, dried, mounted on card.

\section{Locality label}

Venezuela Aragua / Rancho Grande / 1100 m 11.I.75.

\section{Taxonomy}

Spongiphoridae, Sparattinae, Sparattini, Sparatta spiculifera (Brindle, 1977).

\section{Remark}

Transferred by Steinmann (1989b: 155). 


\section{spinosa Brindle, 1966 (Allodahlia)}

Allodahlia spinosa Brindle, 1966h: 444, figs 7-9, 11-12.

\section{Type material}

1 o, paratype, F3233.1696, dried, mounted on card.

\section{Locality label}

Pt Bango / Mindanao.

\section{Taxonomy}

Forficulidae, Allodahliinae, Allodahlia ancylura (Dohrn, 1865).

\section{Remark}

Allodahlia spinosa Brindle, 1966 was synonymised with Allodahlia ancylura (Dohrn, 1865) by Brindle (1972b: 29).

\section{srivastavai Brindle, 1981 (Capralabia)}

Capralabia srivastavai Brindle, 1981: 79.

\section{Type material}

1 ô, paratype, F3233.1438, dried, mounted on card.

\section{Locality label}

Panama, Barro / Colorado Is., S-M / light trap, ground / level / 14.11.1978 / Dr. Henk Wolda, STRI.

\section{Taxonomy}

Anisolabididae, Anisolabidinae, Capralabis srivastavai Brindle, 1981.

\section{Remark}

The genus is named Capralabia in the original description.

\section{steinmanni Brindle, 1981 (Capralabis)}

Capralabis steinmanni Brindle, 1981: 80.

\section{Type material}

1 , paratype, F3233.1439, dried, mounted on card.

\section{Locality label}

Venezuela Ara- / gua, El Limon. / 450 m 13-IX-1978.

\section{Taxonomy}

Anisolabididae, Anisolabidinae, Capralabis steinmanni Brindle, 1981.

\section{Remark}

The original description refers to a second female paratype at MMUE. 


\section{stemmleri Brindle, 1975 (Diplatys)}

Diplatys stemmleri Brindle, 1975c: 10, figs 4-6.

\section{Type material}

1 , paratype, F3233.1310, dried, mounted on card.

\section{Locality label}

21 km O Wangdi / Phodr. 1700 - 2000 / Nat.-Hist. Museum / Basel - Bhutan / Expedition 1972.

\section{Taxonomy}

Diplatyidae, Diplatyinae, Haplodiplatys stemmleri (Brindle, 1975).

\section{Remarks}

1. Transferred by Steinmann (1982a: 211).

2. The original description gives the locality as Wangdiphodrang and the date as 15 Jun. 1972.

\section{straeleni Hincks, 1955 (Apolabis)}

Apolabis straeleni Hincks, 1955b: 7, figs 1-2.

\section{Type material}

1 ${ }^{\lambda}$, paratype, F3233.1414; 1 + , paratype, F3233.1315; dried, mounted on card.

\section{Locality labels}

F3233.1414: Congo belge : P.N.U. / Kaswabilenga (r. Dr. Lufira) / (700 m.) 6-9-x-1947 / Mis. G. F. De Witte. 805a.

F3233.1415: Same data except date, 18-23 Sep. 1947, and number, 769a.

\section{Taxonomy}

Anisolabididae, Anisolabidinae, Anisolabis straeleni (Hincks, 1955).

\section{Remarks}

1. Transferred by Brindle (1978: 96).

2. Index card entry: Africa, Zaire, Kaswebilenga, Upemba Nat. Park.

\section{submetallica Brindle, 1966 (Brachylabis)}

Brachylabis submetallica Brindle, 1966e: 40, figs 8, 14.

\section{Type material}

1 ${ }^{\lambda}$, paratype, F3233.1478; 1 \&, paratype, F3233.1479; dried, mounted on card.

\section{Locality labels}

F3233.1478-1479: Récolté dans / racines / de roseaux // I.R.S.A.C. Mus. Congo / Kivu : Terr. Uvira / Rives L. Tanganika / 14-II-1952 / N. Leleup.

\section{Taxonomy}

Anisolabididae, Brachylabidinae, Metisolabis submetallica (Brindle, 1966). 


\section{Remark}

Transferred by Steinmann (1989a: 282).

\section{sumatrana Brindle, 1965 (Allostethella)}

Allostethella sumatrana Brindle, 1965: 591, figs 3, 10, 20.

\section{Type material}

1 +, paratype, F3233.1490, dried, mounted on card.

\section{Locality label}

Sumatra's O K / Sibolangit.

\section{Taxonomy}

Labiduridae, Allostethinae, Allostethella sumatrana Brindle, 1965.

\section{sumatrana Borelli, 1927 (Chaetospania)}

Chaetospania sumatrana Borelli, 1927: 71, figs 1-3.

\section{Type material}

$3 \lesssim \widehat{\jmath}, 3$ 우, syntypes, F3233.1519, dried, mounted on card.

\section{Locality label}

Gunung Singgalang / Sumatra's Westkust / M. 192 / leg. E. Jacobson.

\section{Taxonomy}

Spongiphoridae, Sparattinae, Chaetospaniini, Chaetospania sumatrana Borelli, 1927.

\section{surinamensis Brindle, 1968 (Larex)}

Larex surinamensis Brindle, 1968g: 34, figs 28-29.

\section{Type material}

1 ऽ, paratype, F3233.1554, dried, mounted on card.

\section{Locality label}

Mus. Leiden / D. C. Geÿskes. / Waremapau / 1-VIII-1939.

\section{Taxonomy}

Spongiphoridae, Spongiphorinae, Marava surinamensis (Brindle, 1968).

\section{Remarks}

1. Transferred by Brindle (1971b: 562).

2. Index card entry: Surinam.

3. The locality is given as Waremapan in the original description, not Waremapau (locality label). (Waremapan Kreek, Sipaliwini, is in the southeastern corner of Suriname.) 
sutteri Hincks, 1954 (Labia)

Labia sutteri Hincks, 1954e: 17, figs 8-11.

\section{Type material}

1 ${ }^{1}$, paratype, F3233.668; 1 + , paratype, F3233.667; dried, mounted on card.

\section{Locality labels}

F3233.667-668: O. Sumba / Mau Marru / 19.7.1949 / Buhler \& Sutter.

\section{Taxonomy}

Spongiphoridae, Labiinae, Paralabella sutteri (Hincks, 1954).

\section{Remark}

Transferred by Steinmann (1989b: 488).

\section{tanganyikae Brindle, 1964 (Gelotolabis)}

Fig. 21

Gelotolabis tanganyikae Brindle, 1964: 754, figs 1-2, 15.

\section{Type material}

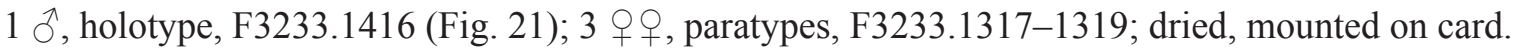

\section{Type locality}

Njombe / 6000-6500 ft. / Tanganyika / I.II.1957 W. P.

\section{Locality labels}

As type locality except date: F3233.1317-1318, 26 Feb. 1957; F3233.1319, 21 Aug. 1952, Dr W. Peters.

\section{Taxonomy}

Anisolabididae, Anisolabidinae, Gonolabis tanganyikae (Brindle, 1964).

\section{Remarks}

1. Transferred by Steinmann (1978: 193).

2. The date of the holotype does not match that given for the material examined by Brindle in the original description, i.e., '26.ii.57 (holotype, allotype)', although the rest of the locality data matches. There is no reference to female paratypes examined except the allotype. The female specimens F3233.13171319 have 'Paratype' on Brindle's determination labels.

\section{taurinus Brindle, 1982 (Diplatys)}

Diplatys taurinus Brindle, 1982b: 163, figs 4-5.

\section{Type material}

1 , , paratype, F3233.1314, dried, mounted on card.

\section{Locality label}

Tanzania / E. Usambara Mts. / Amani 1000m / 20.VII. 1980. 


\section{Taxonomy}

Diplatyidae, Diplatyinae, Diplatys taurinus Brindle, 1982.

\section{tenebrosus Brindle, 1968 (Hamaxas)}

Hamaxas tenebrosus Brindle, 1968h: 546, figs 15, 23.

\section{Type material}

1 O , paratype, F3233.1677; 1 paratype (gender unspecified), F3233.1678; dried, mounted on card.

\section{Locality labels}

F3233.1677-1678: Dapa / Siargao.

\section{Taxonomy}

Chelisochidae, Chelisochinae, Hamaxas tenebrosus Brindle, 1968.

\section{Remark}

The original description refers only to the holotype, allotype and one male paratype, and states that holotype and allotype are in the Riksmuseum, Stockholm.

\section{tibetanus Hincks, 1955 (Diplatys)}

Diplatys tibetanus Hincks, 1955a: 76, figs 81-83.

\section{Type material}

1 Oे, paratype, F3233.1306; 1 \%, paratype, F3233.1311; dried, mounted on pin.

\section{Locality labels}

F3233.1306, F3233.1311: Tibet: / Rongshar Valley. / 9,500ft. 25.vi.1924. / Maj. R. W. G. Hingston.

\section{Taxonomy}

Diplatyidae, Diplatyinae, Haplodiplatys tibetanus (Hincks, 1955).

\section{Remarks}

1. Transferred by Steinmann (1974: 204).

2. The original description has the date 24 Jun. 1924, not 25 Jun. 1924. Both specimens have the word 'Paratype' on Hincks' determination labels.

\section{tibialis Hincks, 1953 (Chaetospania)}

Chaetospania tibialis Hincks, 1953: 371, figs 10-12.

\section{Type material}

1 ऽ, paratype, F3233.1525, dried, mounted on card.

\section{Locality label}

Madagascar C. / Périnet 8.46 / Prof. J. Millot. 


\section{Taxonomy}

Spongiphoridae, Sparattinae, Chaetospaniini, Chaetospania tibialis Hincks, 1953.

\section{tigrina Brindle, 1967 (Echinosoma)}

Echinosoma tigrina Brindle, 1967b: 155, figs 18-21.

\section{Type material}

1 §ै, paratype, F3233.87; 1 q, paratype, F3233.88; dried, mounted on pin.

\section{Locality labels}

F3233.87-88: Long / Navang / O. Borneo / Mjöberg.

\section{Taxonomy}

Pygidicranidae, Echinosomatinae, Echinosoma tigrina Brindle, 1967.

$$
\text { townesi Brindle, } 1979 \text { (Marava) }
$$

Marava townesi Brindle, 1979: 152, fig. 2.

\section{Type material}

1 ऽ, paratype, F3233.1555, dried, mounted on card.

\section{Locality label}

Guatemala / 371656 / Primavera logs / under bark / N.Y. Jan. 18, 1937.

\section{Taxonomy}

Spongiphoridae, Spongiphorinae, Marava townesi Brindle, 1979.

\section{transversa Brindle, 1978 (Isolabis)}

Isolabis transversa Brindle, 1978: 41, figs 35-36.

\section{Type material}

1 ऽ, paratype, F3233.1480, dried, mounted on card.

\section{Locality label}

Ilot for. maréc. / avec Pandanus // I.R.S.A.C.-Mus. Congo / Kwango: terr. De Feshi, / rive dr. Kwenge IV-1959 / B. 78 Mme J. Leleup.

\section{Taxonomy}

Anisolabididae, Isolabidinae, Isolabis transversa Brindle, 1978.

\section{Remark}

The original description gives the date as Mar. 1959, not Apr. 1959 (locality label).

\section{triangulata Hincks, 1950 (Forficula)}

Forficula triangulata Hincks, 1950a: 180, figs 3-4. 
Type material

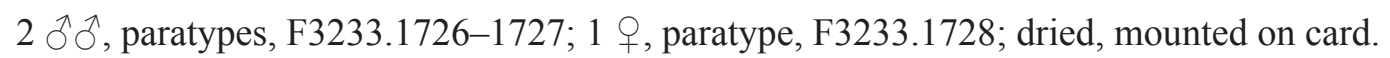

\section{Locality labels}

F3233.1726-1728: Kilimanjaro T.T. / 14,500 ft. / 27.X.48 Dr. G. Salt. // Under \& beside stones on saddle.

\section{Taxonomy}

Forficulidae, Forficulinae, Guanchia triangulata (Hincks, 1950).

\section{Remark}

Transferred by Steinmann (1993: 561).

triangulatus Brindle, 1987 (Haplodiplatys)

Haplodiplatys triangulatus Brindle, 1987: 335, figs 1-3.

\section{Type material}

1 ऽે, paratype, F3233.1315, dried, mounted on card.

\section{Locality label}

E. Nepal: / Arun Valley / VI-15-1954 / 7,000ft. L. Swan.

\section{Taxonomy}

Diplatyidae, Diplatyinae, Haplodiplatys triangulatus Brindle, 1987.

\section{trinodosa Brindle, 1967 (Gonolabina)}

Fig. 23

Gonolabina trinodosa Brindle, 1967c: 9, figs 2, 4.

\section{Type material}

1 d , holotype, F3233.1454 (Fig. 23), dried, mounted on card. 1 \%, allotype, F3233.1455, dried, mounted on pin.

\section{Type locality}

Tragadero / (cerca Jauja) / 3500 m / 3.IV.49 / Coll. Blancas

\section{Locality label}

F3233.1455: Acolla (cerca / Jauja) 3460 m / VIII.49 / Coll: F. Blancas.

\section{Taxonomy}

Anisolabididae, Gonolabininae, Gonolabina trinodosa Brindle, 1967.

\section{Remark}

The original description has the altitude $3600 \mathrm{~m}$ for the allotype, not $3460 \mathrm{~m}$. 


\section{trinodosa Brindle, 1977 (Pygidicrana)}

Pygidicrana trinodosa Brindle, 1977b: 111, figs 1-3.

\section{Type material}

1 ठ, paratype, F3233.1319, dried, mounted on card.

\section{Locality label}

Curimagua / Venezuela, Falcon / 16.VIII.1975 / R. E. Dietz. / Leg.

\section{Taxonomy}

Pygidicranidae, Pygidicraninae, Pygidicrana trinodosa Brindle, 1977.

\section{Remark}

The original description has the date 16 Dec. 1975, not 16 Aug. 1975.

\section{trituberculatus Brindle, 1971 (Purex)}

Purex trituberculatus Brindle, 1971a: 171, fig. 24.

\section{Type material}

1 Oे, paratype, F3233.1598, dried, mounted on pin.

\section{Locality label}

Venezuela: Ar. / Rancho Grande / 1100m 16-23.X.66.

\section{Taxonomy}

Spongiphoridae, Spongiphorinae, Purex sinuatus Brindle, 1971.

\section{Remark}

Purex trituberculatus Brindle, 1971 was synonymised with P. sinuatus Brindle, 1971 by Brindle (1977b: 119).

\section{trituberculatus Brindle, 1973 (Spongovostox)}

Spongovostox trituberculatus Brindle, 1973f: 186, figs 249, 256.

\section{Type material}

1 o, paratype, F3233.1612, dried, mounted on card.

\section{Locality label}

Coll. Mus. Congo / Kivu : Sanghe, Pl. De / Ruzizi (A la lumière) / XII-1951 / H. Bomans.

\section{Taxonomy}

Spongiphoridae, Spongiphorinae, Spongovostox trituberculatus Brindle, 1973.

\section{truncata Brindle, 1978 (Euborellia)}

Euborellia truncata Brindle, 1978: 175, figs 291, 322. 
Type material

1 đ̊, paratype, F3233.1442; 1 q, paratype, F3233.1443; dried, mounted on pin.

\section{Locality labels}

F3233.1442-1443: Uganda. / Entebbe. / v - vi 1912 / Mrs. Gowdey.

\section{Taxonomy}

Anisolabididae, Anisolabidinae, Euborellia truncata Brindle, 1978.

\section{tuberculata Hincks, 1958 (Isolaboides)}

Isolaboides tuberculata Hincks, 1958b: 133, figs 1-2.

\section{Type material}

1 Oे, paratype, F3233.1484, dried, mounted on card.

\section{Locality label}

Liban: Les Cedres / Coiffait. 28.IX. / Museum Paris.

\section{Taxonomy}

Spongiphoridae, Isolaboidinae, Isolaboides tuberculata Hincks, 1958.

\section{Remark}

Index card entry: Lebanon.

\section{tuberosa Brindle, 1970 (Sphingolabis)}

Sphingolabis tuberosa Brindle, 1970f: 678, figs 47-49.

\section{Type material}

1 Sิ, paratype, F3233.695, dried, mounted on card.

\section{Locality label}

Solomon Is. / Guadacanal [sic] / Popamanasiu / 25. X. 1965 / P. Greenslade.

\section{Taxonomy}

Spongiphoridae, Labiinae, Sphingolabis tuberosa Brindle, 1970.

\section{umbrosa Brindle, 1964 (Gelotolabis)}

Fig. 20

Gelotolabis umbrosa Brindle, 1964: 752, figs 5, 13.

\section{Type material}

1 ô, holotype, F3233.1420 (Fig. 20); 1 ڤึ, paratype, F3233.1421; dried, mounted on pin.

\section{Type locality}

Tanganyika / Nachingwea / Southern Prov. / IX. 53 - III. 54 / V. F. Eastop. 


\section{Locality label}

F3233.1421: As type locality.

\section{Taxonomy}

Anisolabididae, Anisolabidinae, Gonolabis umbrosa (Brindle, 1964).

\section{Remark}

Transferred by Steinmann (1978: 195).

\section{uncinata Brindle, 1978 (Anisolabis)}

Anisolabis uncinata Brindle, 1978: 88, figs 110-112, 236.

\section{Type material}

1 ऽ, paratype, F3233.1422, dried, mounted on card.

\section{Locality label}

Biot. No 15 / marais boisés / avec Pandanus. I.R.S.A.C. - Mus. Congo / Kivu : Mwenga / 1250 m. 24IV-1958 / B.15 N. Leleup.

\section{Taxonomy}

Anisolabididae, Anisolabidinae, Flexiolabis uncinata (Brindle, 1978).

\section{Remark}

Transferred by Steinmann (1989a: 35).

\section{unicolor Brindle, 1975 (Brachylabis)}

Brachylabis unicolor Brindle, 1975b: 684, figs 1-2.

\section{Type material}

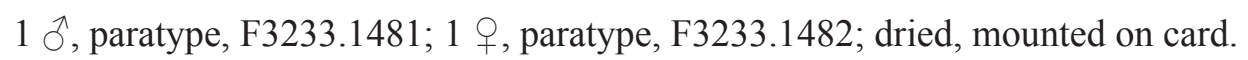

\section{Locality labels}

F3233.1481: Tanzanie: Mts Uluguru / Kimboza for. héliophile / alt. 600m 24-30/VII/71 // Coll. Mus. Tervuran / Mission Mts Uluguru / L. Berger, N. Leleup / J. Debecker V/VIII/71.

F3233.1482: Tanzanie: Mts Uluguru / Chenzema, alt. 1700m. / 2-22/VII/71 // Coll. Mus. Tervuran / Mission Mts Uluguru / L. Berger, N. Leleup / J. Debecker V/VIII/71.

\section{Taxonomy}

Anisolabididae, Isolabidinae, Isolabis unicolor (Brindle, 1975).

\section{Remarks}

1. Transferred by Brindle (1978: 44).

2. The original description states that all paratypes have the locality Kimboza, as for F3233.1481. The locality data on the label of the female (F3233.1482) corresponds exactly to the allotype data in the description. 


\section{unicolor Brindle, 1966 (Emboros)}

Emboros unicolor Brindle, 1966g: 255, figs 62, 65, 69.

\section{Type material}

4 우, paratypes, F3233.1688-1691, dried, mounted on card.

\section{Locality labels}

F3233.1688-1691: Madagascar-Est / dct. Sambava / R. N. XII / Marojejy-Ouest 1140m / XI-59 P. Soga.

\section{Taxonomy}

Forficulidae, Skendylinae, Cosmiella unicolor (Brindle, 1966).

\section{Remark}

Transferred by Steinmann (1989c: 675).

\section{vansomereni Brindle, 1969 (Chaetospania)}

Chaetospania vansomereni Brindle, 1969a: 98, figs 1-2.

\section{Type material}

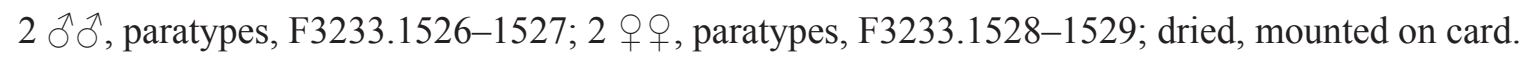

\section{Locality labels}

F3233.1526-1529: Van Someren / Meru, 7/43.

\section{Taxonomy}

Spongiphoridae, Sparattinae, Chaetospaniini, Chaetospania vansomereni Brindle, 1969.

\section{Remark}

Index card entry: Tanzania.

\section{variabilis Brindle, 1973 (Mixocosmia)}

Mixocosmia variabilis Brindle, 1973b: 50, figs 1-3.

\section{Type material}

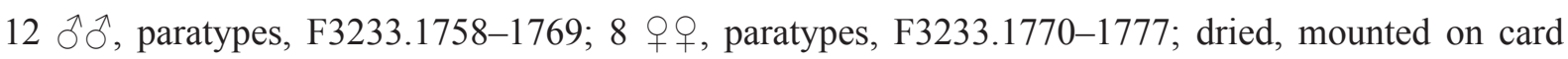
except: F3233.1759, 1762-1763, 1766, 1769-1770, 1772-1773; dried, mounted on pin.

\section{Locality labels}

F3233.1762: Venezuela - Aragua / Rancho Grande / 1100m. 10-VIII-69 // J. A. Clavijo / col. Same data except date: F3233.1765, 11 Aug. 1969; F3233.1759, 20 Aug. 1969; F3233.1758, F3233.1773, 27 Aug. 1969.

Same data except date and collector: F3233.1770, 30-VII-69 // J. Salcedo / J. A. Clavijo; F3233.1766, 5-IX-69 // J. Salcedo / J. A. Clavijo; F3233.1768, 16.V.70 // F. Fernandez Y. / J. A. Clavijo; F3233.1772, 14-VIII-49 // F. Fernandez Y / - P Fenjues; F3233.1763, 20.VIII.69 // F. Fernandez Y. / C. J. Rosales / Cols; F3233.1771, 7.XI.55 // F. Fernandez Y. / C. J. Rosales / Cols; F3233.1761: [9?].VI.55 // F. Fernandez Y. / C. J. Rosales / Cols; F3233.1760, 4-IV-51 / C. J. Rosales; F3233.1767, F3233.1769, 
F3233.1774-1777, 17.XI.1968 / H. Reichardt col; F3233.1764, as F3233.1768 except 21.V.71 // En la / Luz.

\section{Taxonomy}

Forficulidae, Skendylinae, Mixocosmia variabilis Brindle, 1973.

\section{Remarks}

1. Steinmann (1989c) and Hopkins et al. (2014) have Brindle's original name as Kleter variabilis and so have the current name as Mixocosmia variabilis (Brindle, 1973); however, Brindle's original description names the new species Mixocosmia variabilis, not Kleter.

2. The number of paratypes does not match that stated in the original description, i.e., of 36 male and 62 female paratypes with the same data as the holotype (dates ranging from 30 Apr. 1969 to 26 May 1971) mentioned in the description, 11 male and 14 female paratypes were at MMUE, not 12 males and 8 females.

\section{variabilis egoloensis Brindle, 1970 (Auchenomus)}

Auchenomus variabilis egoloensis Brindle, 1970f: 667, figs 23-24.

\section{Type material}

1 đ̊, paratype, F3233.559; 1 +, paratype, F3233.560; dried, mounted on card.

\section{Locality labels}

F3233.559-560: Solomon Is. / New Georgia Group / nr. Egolo 1-25 m. / 16-VII-1959.

\section{Taxonomy}

Spongiphoridae, Sparattinae, Auchenomini, Auchenomus variabilis egoloensis Brindle, 1970.

\section{variabilis guadalcanalensis Brindle, 1970 (Auchenomus)}

Fig. 49

Auchenomus variabilis guadalcanalensis Brindle, 1970f: 668, figs 25-26.

\section{Type material}

1 §, holotype, F3233.557 (Fig. 49); 1 q, allotype, F3233.558; dried, mounted on card.

\section{Type locality}

Solomon Is. / Guadalcanal / Rua Vata / 21:xi:1954 / E S Brown.

\section{Locality label}

F3233.558: As type locality.

\section{Taxonomy}

Spongiphoridae, Sparattinae, Auchenomini, Auchenomus variabilis guadalcanalensis Brindle, 1970.

$$
\text { variegatus Hincks, } 1957 \text { (Blandex) }
$$

Blandex variegatus Hincks, 1957b: 45, figs 7-10. 
Type material

1 , paratype F3233.1338, dried, mounted on card.

\section{Locality label}

S. Afr. Cape Prov. / Cape Point / Nature Reserv / 24.X.50 No. 15. Swedish South Africa / Expedition / 1950-1951 / Brinck-Rudebeck.

\section{Taxonomy}

Pygidicranidae, Blandicinae, Blandex variegatus Hincks, 1957.

venezuelica Brindle, 1977 (Marava)

Marava venezuelica Brindle, 1977b: 123, figs 25-26.

\section{Type material}

1 đ̊, paratype, F3233.1556; 1 \&, paratype, F3233.1557; dried, mounted on card.

\section{Locality labels}

F3233.1556: Venezuela Aragua / Rancho Grande / 1100 m. 16.VIII.74.

F3233.1557: Same data except date 4 Aug. 1974.

\section{Taxonomy}

Spongiphoridae, Spongiphorinae, Marava venezuelica Brindle, 1977.

\section{Remark}

The original description gives the date for the male paratype (F3233.1556) as 2 Aug. 1974, not 16 Aug. 1974.

\section{venezuelica Brindle, 1982 (Yepezia)}

Yepezia venezuelica Brindle, 1982a: 38, figs 7-11.

\section{Type material}

1 ${ }^{\lambda}$, paratype, F3233.1636, dried, mounted on card, without head.

\section{Locality label}

Güigüe / Venezuela - Cara- / bobo m. / 23.II.1968. // J. \& B. Bechyne / leg.

\section{Taxonomy}

Spongiphoridae, Geracinae, Yepezia venezuelica Brindle, 1982.

venezuelicum Brindle, 1974 (Barygerax)

Barygerax venezuelicum Brindle, 1974b: 114, figs 5-8.

\section{Type material}

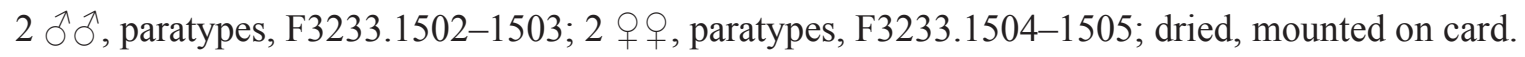




\section{Locality labels}

F3233.1502: Venezuela - Aragua / Rancho Grande / 1100 m. 25-VII-69. // J. Salcedo / col. // Venezuela Inst. / Zool. Agricola - / Fac. Agronomia / Univ. Central.

Same data except date: F3233.1503, 30 Jul. 1969; F3233.1504, 10 Aug. 1969; F3233.1505, 25 Mar. 1971.

\section{Taxonomy}

Spongiphoridae, Geracinae, Barygerax venezuelicum Brindle, 1974.

\section{Remarks}

1. The original description has the date 30 Aug. 1969 for the specimen corresponding to F3233.1503, not 30 Jul. 1969.

2. There are two male and two female paratypes at MMUE, not three males and one female as in the description.

\section{venezuelicus Brindle, 1973 (Kleter)}

Kleter venezuelicus Brindle, 1973b: 49, figs 5-6.

\section{Type material}

1 ふै, paratype, F3233.1736; 2 우, paratypes, F3233.1737-1738; dried, mounted on card.

\section{Locality labels}

F3233.1736: Venezuela - Ara- / gua- El Limon. / 450m. 22-I-70. // En Pseudo tallos de camburusados / como trampa. // col. P. Bolivar.

F3233.1737: Venezuela - Ara- / gua - Maracay / 450m. 19-I-70. // En tramp / de cambur. // col. P. Bolivar. F3233.1738: Venezuela - Cara- / bobo - Mariara / 30-I-1970. // Trampa / de Luz.

\section{Taxonomy}

Forficulidae, Skendylinae, Hypocosmiella venezuelica (Brindle, 1973).

\section{Remarks}

Transferred by Steinmann (1993: 184). Not listed in Hopkins et al. (2014).

\section{vittipennis Hincks, 1955 (Cranopygia)}

Cranopygia vittipennis Hincks, 1955c: 820, figs 13-14.

\section{Type material}

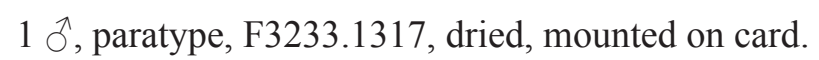

\section{Locality label}

S. India: S. Coorg / Ammatti 3100 ft. / 3.XI.52 under bark / P.S. Nathan.

\section{Taxonomy}

Pygidicranidae, Pygidicraninae, Acrania vittipennis (Hincks, 1955). 


\section{Remarks}

Cranopygia vittipennis Hincks was transferred to Epicranopygia by Steinmann (1986b: 271). The species was transferred to Acrania Burr by Engel \& Haas (2007: 19).

\section{weidneri Brindle, 1966 (Esphalmenus)}

Esphalmenus weidneri Brindle, 1966f: 128, figs 9, 11-12.

\section{Type material}

1 o, paratype, F3233.1365, dried, mounted on card.

\section{Locality label}

Probably near / Santiago / Chile / Dr. G. Rahm.

\section{Taxonomy}

Pygidicranidae, Esphalmeninae, Esphalmenus weidneri Brindle, 1966.

\section{wittei Hincks, 1955 (Diaperasticus)}

Diaperasticus wittei Hincks, 1955b: 12, figs 4-5.

\section{Type material}

1 ô, paratype, F3233.1713; 1 + , paratype, F3233.1714; dried, mounted on card.

\section{Locality labels}

F3233.1713: Congo belge: P. N. U. / Lusinga (1760 m.) / 18-vii 1947 / Mis. G. F. De Witte. 604a. F3233.1714: Congo belge : P. N. U. / Kalule N. r. g. Face Mujinga- / Kalenge (1050m.) 28-II-3-III-49 / Mis. G. F. De Witte. 2399a.

\section{Taxonomy}

Forficulidae, Diaperasticinae, Diaperasticus wittei Hincks, 1955.

\section{wittmeri Brindle, 1975 (Irdex)}

Irdex wittmeri Brindle, 1975c: 28, figs 24-25.

\section{Type material}

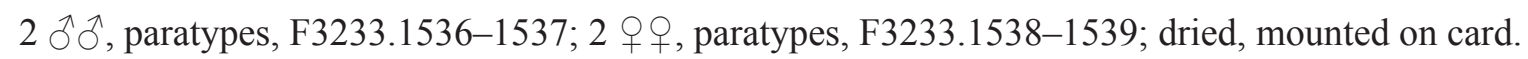

\section{Locality labels}

F3233.1536: Wangdi Phodrang / 1300m Darju La. // Nat.-Hist. Museum / Basel - Bhutan / Expedition 1972.

F3233.1537-1538: Km 87 von / Phuntsholing 22/5 / 1680m. Nat.-Hist. Museum / Basel - Bhutan / Expedition 1972.

F3233.1539: 21 km O Wangdi / Phodr. 1700-2000. Nat.-Hist. Museum / Basel - Bhutan / Expedition 1972. 


\title{
Taxonomy
}

Spongiphoridae, Spongiphorinae, Irdex wittmeri Brindle, 1975.

\author{
woldai Brindle, 1988 (Mixocosmia)
}

Mixocosmia woldai Brindle, 1988: 55, figs 25-27.

\section{Type material}

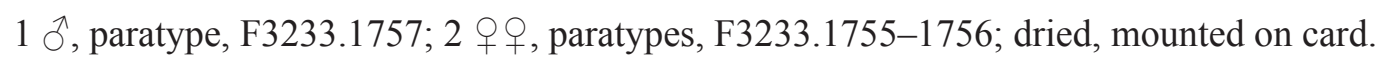

\section{Locality labels}

F3233.1755-1756: Panama, Fortuna / Wet forest / at light, 1050m / 29.6.1979 / Dr. Henk Wolda, STRI. F3233.1757: Same data except date 25 Sep. 1978.

\section{Taxonomy}

Forficulidae, Skendylinae, Mixocosmia woldai Brindle, 1988.

\section{wuermlii Brindle, 1975 (Spongovostox)}

Spongovostox wuermlii Brindle, 1975c: 33, figs 33-34.

\section{Type material}

1 ऽ, paratype, F3233.1613, dried, mounted on card.

\section{Locality label}

km 87 von 1680 m / Phuntsholing 22/5. // Basel-Bhutan / Expedition 1972.

\section{Taxonomy}

Spongiphoridae, Spongiphorinae, Spongovostox wuermlii Brindle,1975.

$$
\text { yepezi Brindle, } 1974 \text { (Cylindrogaster) }
$$

Cylindrogaster yepezi Brindle, 1974b: 108, figs 1-2.

\section{Type material}

1 Oे, paratype, F3233.1312, dried, mounted on pin.

\section{Locality label}

Venezuela - Aragua / Rancho Grande / 1100m 27.III.67 / C. J. Rosales J. Salcedo.

\section{Taxonomy}

Pygidicranidae, Cylindrogastrinae, Cylindrogaster yepezi Brindle, 1974. 
Other Material: Specimens carrying a type label, but for which no description has been found

These taxa are not listed in Steinmann (1989c) or Hopkins et al. (2014) and the present author has been unable to locate any published descriptions. Thus, these species names seem to represent unpublished 'manuscript names' only.

\section{flavolineata Brindle (Tagalina) MS}

Figs 62-63

\section{Type material}

1 Oे, paratype, F3233.1864 (Fig. 62), dried, mounted on pin.

\section{Locality label}

Museum Leiden / Nieuw Guinea Exp K.N.A.G. 1939 / Araboebirak 11-x-1939.

\section{Determination label}

Manchester Museum Paratype // Tagalina đ̊ / flavolineata Brindle / det. A. Brindle 1980 / paratype.

\section{Taxonomy}

Pygidicranidae, Pygidicranidae, Tagalina.

\section{Remarks}

Found recently in a box separate from the collection. A note written by A. Brindle pinned next to the specimen reads "Tagalina flavolineata n. sp. (paratype for Manchester) (holotype in Leiden). Not yet described (paper not written)" (Fig. 63).

The holotype and allotype have been confirmed to be at the Naturalis Biodiversity Center, Leiden (L. Willemse, pers. comm.), labelled Tagalina flavolineata and bearing Brindle's determination label.

\section{rossi Hincks (Carcinophora) MS}

Fig. 61

\section{Type material}

1 Oे, paratype, F3233.1848 (Fig. 61), dried, mounted on pin.

\section{Locality label}

PERU: 43 mi. E. / Tingo Maria / 1200 m. XI.18.54. // E. I. Sehlinger / \& E. S. Ross / collectors.

\section{Determination label}

Carcinophora / rossi Hincks / TYPE o / det. W. D. Hincks // MS name.

\section{Taxonomy}

Anisolabididae, Anisolabidinae, Carcinophora.

\section{spatulus Brindle (Nesogaster)}

Fig.59

\section{Type material}

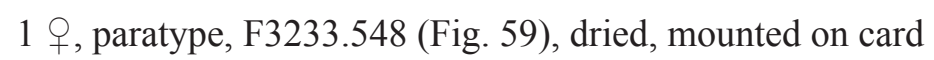

\section{Locality label}

Fiji, Vanua Levu / Nandrau / 9.10.1977 / G. Kuschel. 
Determination label

Nesogaster + , / spatulus Brindle / det. A. Brindle 1983 / paratype.

\section{Taxonomy}

Spongiphoridae, Nesogastrinae, Nesogaster.

\section{Remarks}

This species is fully described in a typewritten manuscript in the Brindle archive, item 65 (Brindle \& Maddison: date not known [unpublished?]), as ' $N$. spatulus sp. n. NZAC Endemic' [NZAC = New Zealand Arthropod Collection]. The label data matches the described material exactly.

\section{subcarinata Brindle (Metalabis) MS}

Fig. 60

\section{Type material}

1 Oे, holotype, F3233.1834 (Fig. 60), dried, mounted on card.

\section{Type locality}

Brokopondo / Surinam / 20.IX.1963.

\section{Taxonomy}

Anisolabididae, Anisolabidinae, Metalabis.

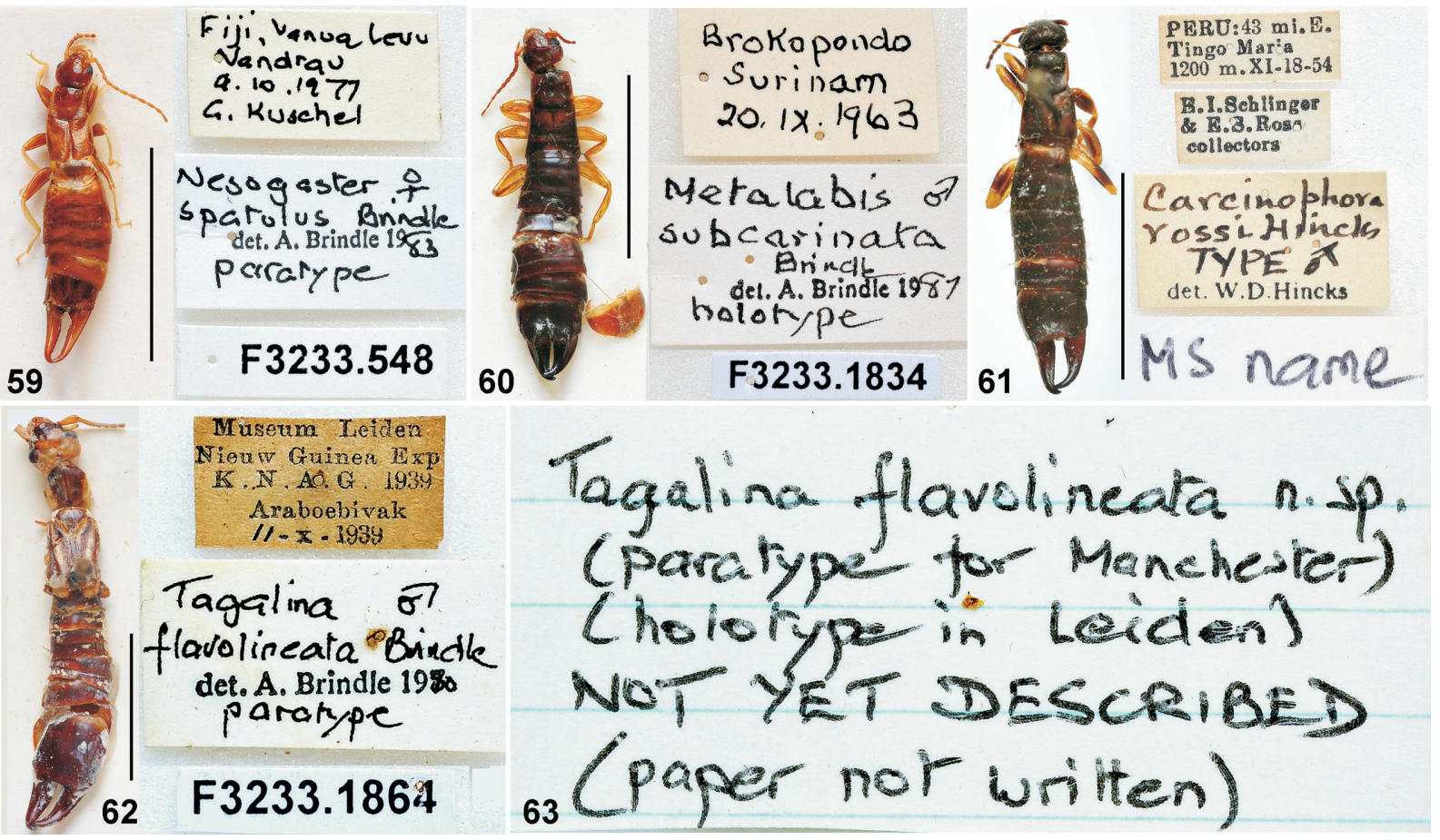

Figs 59-63. Other material, presumed manuscript names, in the collection of the Manchester Museum. 59. Nesogaster spatulus Brindle (det. 1983). 60. Metalabis subcarinata Brindle (det. 1987). 61. Carcinophora rossi Hincks (no date). 62. Tagalina flavolineata Brindle (det. 1980). 63. Brindle's handwritten label for the Tagalina flavolineata specimen (Fig. 62). Scale bars $=1 \mathrm{~cm}$. 


\section{Acknowledgements}

I wish to thank Dmitri Logunov (Manchester Museum, UK) for the opportunity to work on this collection, his advice, proofreading, and processing the holotype photographs; Philip Rispin (Manchester Museum, UK) for the use of his equipment, time and skill in photographing the specimens; Leonid Anisyutkin (Zoological Institute RAS, St. Petersburg, Russia) for advice on the taxonomy of the type material; Mary Angela Nellie Jayapaul for information relating to the life of P.S. Nathan; Luc Willemse (Naturalis Biodiversity Center, Leiden, Netherlands) for information relating to Brindle MS species names; and the two anonymous referees and desk editor Danny Eibye-Jacobsen (EJT) for their detailed and helpful comments.

\section{References}

Alberti S.J.M.M. 2009. Nature and Culture. Objects, Disciplines and the Manchester Museum. Manchester University Press.

Ashmole P. \& Ashmole M. 2004. The invertebrates of Prosperous Bay Plain, St Helena. (.doc file). St Helena and Ascension Island Natural History. Available from http://www.kidstonmill.org.uk [accessed 23 Feb. 2015]

Borelli A. 1900. Descrizione di una nuova Forficula del Congo. Bollettino dei Musei di Zoologia ed Anatomia comparata della Reale Università di Torino 15 (381): 1-3. Available from http://www. biodiversitylibrary.org/item/43398\#page/183/mode/1up [accessed 12 Aug. 2015]

Borelli A. 1906. Forficole di Costa Rica. Bollettino dei Musei di Zoologia ed Anatomia comparata della Reale Università di Torino 21 (531): 1-19. Available from http://www.biodiversitylibrary.org/ item/43046\#page/111/mode/1up [accessed 12 Aug. 2015]

Borelli A. 1907a. Nuova Forficola dell'Ecuador. Bollettino dei Musei di Zoologia ed Anatomia comparata della Reale Università di Torino 22 (552): 1-3. Available from http://www.biodiversitylibrary.org/ item/43468\#page/33/mode/1up [accessed 12 Aug. 2015]

Borelli A. 1907b. Spedizione al Ruwenzori di S.A.R. Luigi Amedeo di Savoia Duca degli Abruzzi. XVII. Nuove specie di Forficole. Bollettino dei Musei di Zoologia ed Anatomia comparata della Reale Università di Torino 22 (558): 1-6. Available from http://www.biodiversitylibrary.org/ item/43468\#page/93/mode/1up [accessed 12 Aug. 2015]

Borelli A. 1907c. Spedizione al Ruwenzori di S.A.R. Luigi Amedeo di Savoia Duca degli Abruzzi. XXVII. Nuova specie di Forficule. Bollettino dei Musei di Zoologia ed Anatomia comparata della Reale Università di Torino 22 (572): 1-3. Available from http://www.biodiversitylibrary.org/ item/43468\#page/203/mode/1up [accessed 12 Aug. 2015]

Borelli A. 1909. Nuove forficole del Kashmir (India). Bollettino dei Musei di Zoologia ed Anatomia comparata della Reale Università di Torino 24 (603): 1-4. Available from http://www.biodiversitylibrary. org/item/51282\#page/59/mode/1up [accessed 12 Aug. 2015]

Borelli A. 1927. Fauna sumatrensis. No. 36. Dermaptera. Supplementa Entomologica 15: 69-80.

Brindle A. 1964. A revision of the African species of the genus Gelotolabis Zacher (Dermaptera: Carcinophoridae). Annals and Magazine of Natural History 13 (7): 747-755. http://dx.doi. org/10.1080/00222936408651528

Brindle A. 1965. A revision of the subfamily Allostethinae (Dermaptera, Labiduridae). Annals and Magazine of Natural History 13 (8): 575-596. http://dx.doi.org/10.1080/00222936508651618 
Brindle A. 1966a. A key to the Neotropical genus Idolopsalis Borelli (Dermaptera: Carcinophoridae). Proceedings of the Royal Entomological Society of London B 35: 143-148. http://dx.doi. org/10.1111/j.1365-3113.1966.tb00516.x

Brindle A. 1966b. A new species of Forficula Linnaeus (Dermaptera, Forficulidae) from Africa. Entomologist's Monthly Magazine 102: 147-149.

Brindle A. 1966c. A revision of the Dermaptera of the Congo. Revue de Zoologie et de Botanique Africaines 73 (1-2): 40-58.

Brindle A. 1966d. A revision of the subfamily Labidurinae (Dermaptera, Labiduridae). Annals and Magazine of Natural History 13 (9): 239-269. http://dx.doi.org/10.1080/00222936608656050

Brindle A. 1966e. Dermaptera from Central and East Africa. Revue de Zoologie et de Botanique Africaines 74 (1-2): 29-49.

Brindle A. 1966f. Notes on Dermaptera in the Hamburg Museum. Entomologische Mitteilungen aus dem Zoologischen Museum Hamburg 3 (56): 127-141.

Brindle A. 1966g. The Dermaptera of Madagascar. Transactions of the Royal Entomological Society of London 118: 221-259. http://dx.doi.org/10.1111/j.1365-2311.1966.tb02305.x

Brindle A. 1966h. The Dermaptera of the Naturhistoriska Riksmuseum, Stockholm. I. Arkiv för Zoologi 18 (18): 437-447.

Brindle A. 1967a. Further notes on Dermaptera in the Hamburg Museum. Entomologische Mitteilungen aus dem Zoologischen Museum Hamburg 3 (59): 189-195.

Brindle A. 1967b. The Dermaptera of the Naturhistoriska Riksmuseum, Stockholm. II. Arkiv för Zoologi 20 (7): 147-163.

Brindle A. 1967c. Two new species of Gonolabina Verhoeff (Dermaptera, Carcinophoridae) from Peru. Entomologist's Monthly Magazine 103: 5-11.

Brindle A. 1968a. A new Brazilian species of the genus Brachylabis Dohrn (Dermaptera, Carcinophoridae). Papéis Avulsos de Zoologia, São Paulo 22 (4): 31-34.

Brindle A. 1968b. A new genus and species of blind Dermaptera from the Galapagos Islands. Mission zoologique belge aux Îles Galapagos et en Ecuador 1: 171-176.

Brindle A. 1968c. A revision of the Labiidae (Dermaptera) of the Neo-tropical and Nearctic Regions. 1. Pericominae, Strongylopsalinae, Sparattinae. Journal of Natural History 2: 273-303. http://dx.doi. org/10.1080/00222936800770931

Brindle A. 1968d. Contributions à la connaissance de la faune entomologique de la Côte-d'Ivoire (J. Decelle, 1961-1964). Annales du Musée royal de l'Afrique Centrale, Zoologie (8) 165: 11-20.

Brindle A. 1968e. Dermaptera. Exploration du Parc national de la Garamba 53 (2): 17-32.

Brindle A. 1968f. Notes on the genus Timomenus Burr (Dermaptera, Forficulidae). Entomologist's Monthly Magazine 104: 243-249.

Brindle A. 1968g. The Dermaptera of Surinam and other Guyanas. Studies on the Fauna of Suriname and other Guyanas 10 (36): 1-60.

Brindle A. 1968h. The Dermaptera of the Naturhistoriska Riksmuseum, Stockholm. III. Arkiv för Zoologi 20 (25): 533-552.

Brindle A. 1969a. A key to the African species of the genus Chaetospania Karsch (Dermaptera: Labiidae). Proceedings of the Royal Entomological Society of London B 38 (7-8): 95-100. http://dx.doi. org/10.1111/j.1365-3113.1969.tb00237.x 
Brindle A. 1969b. A new species of Brachylabis Dohrn (Dermaptera, Carcinophoridae) from Malaysia. Entomologist 102: 197-200.

Brindle A. 1969c. Anew species of Pseudovostox Borelli (Dermaptera, Labiidae) from Angola. Opuscula Entomologica 34: 145-150.

Brindle A. 1969d. A new species of Vandex Burr (Dermaptera: Labiidae) from Africa. Entomologist 102: 190-193.

Brindle A. 1969e. Notes on the genus Acanthocordax Günther (Dermaptera, Forficulidae). Entomologist's Monthly Magazine 105: 273-277.

Brindle A. 1970a. A key to the Neotropical Genus Neolobophora Scudder, and related genera (Dermaptera, Forficulidae). Entomologist's Monthly Magazine 106: 97-105.

Brindle A. 1970b. A preliminary revision of the genus Skalistes Burr (Dermaptera, Forficulidae). Entomologist 103: 217-228.

Brindle A. 1970c. Dermaptera. In: Basilewsky P. (ed.). La faune terrestre de l'Île de Sainte-Hélène. Première partie. Annales du Musée royal de l'Afrique Centrale, Zoologie (8) 181: 213-227.

Brindle A. 1970d. Dermaptera from Gabon. Biología Gabonica 6 (1): 11-25.

Brindle A. 1970e. New African species of the genus Pseudovostox Borelli (Dermaptera Labiidae). Revue de Zoologie et de Botanique Africaines 81 (3-4): 255-270.

Brindle A. 1970f. The Dermaptera of the Solomon Islands. Pacific Insects 12 (3): 641-700.

Brindle A. 1970g. The Hemimerina and Dermaptera of Angola. Publicações Culturais da Companhia de Diamantes de Angola 34: 39-74.

Brindle A. 1971a. A revision of the Labiidae (Dermaptera) of the Neo-tropical and Nearctic Regions. II. Geracinae and Labiinae. Journal of Natural History 5: 155-182. http://dx.doi. org/10.1080/00222937100770101

Brindle A. 1971b. A revision of the Labiidae (Dermaptera) of the Neo-tropical and Nearctic Regions. III. Spongiphorinae. Journal of Natural History 5: 521-568. http://dx.doi.org/10.1080/00222937100770391 Brindle A. 1971c. Le Massif des Monts Loma (Sierra Leone). IX. Dermaptera. Mémoires de l'Institut Français d'Afrique Noire 86: 265-281.

Brindle A. 1971d. New Neotropical Species of Dermaptera. Entomologist 104: 249-257.

Brindle A. 1971e. The Dermaptera (Earwigs) of Dominica. Smithsonian Contributions to Zoology 63: 1-24. http://dx.doi.org/10.5479/si.00810282.63

Brindle A. 1971f. The Dermaptera of the Naturhistoriska Riksmuseum, Stockholm, IV. Entomologisk Tidskrift 92 (1-2): 1-27.

Brindle A. 1971g. Two new species of Dermaptera from Brazil. Papéis Avulsos de Zoologia, São Paulo 23 (20): 165-171.

Brindle A. 1972a. A new species of Diplatys Serville (Dermaptera, Diplatyidae) from Costa Rica. Entomologist 105: 281-283.

Brindle A. 1972b. A review of the genus Allodahlia Verhoeff (Dermaptera, Forficulidae). Entomologist's Monthly Magazine 108: 23-30.

Brindle A. 1972c. Dermaptera. Insects of Micronesia 5 (2): 97-171.

Brindle A. 1973a. A new genus and species of Geracinae (Dermaptera, Labiidae) from Guatemala. Entomologist 106: 153-156. 
Brindle A. 1973b. Dermaptera from Venezuela. Part 1. Ancistrogastrinae (Forficulidae). Revista de la Facultad de Agronomia, Universidad del Zulia 7 (1): 47-54.

Brindle A. 1973c. Dermaptera in the Hamburg Museum. Entomologische Mitteilungen aus dem Zoologischen Museum Hamburg 4 (82): 395-402.

Brindle A. 1973d. Notes on Oriental Geracinae (Dermaptera: Labiidae). Entomologist 106: 137-144.

Brindle A. 1973e. Taxonomic notes on the genus Strongylopsalis Burr (Dermaptera, Labiidae). Journal of Entomology B 42 (1): 11-16. http://dx.doi.org/10.1111/j.1365-3113.1973.tb00049.x

Brindle A. 1973f. The Dermaptera of Africa. Part I. Annales du Musée royal de l'Afrique Centrale, Zoologie (8) 205: 1-335.

Brindle A. 1974a. A new species of Dermaptera (Forficulidae) from Mexican bromeliads. The Entomologist's Record and Journal of Variation 86: 6-8. Available from http://www.biodiversitylibrary. org/item/121152\#page/20/mode/1up [accessed 12 Aug. 2015]

Brindle A. 1974b. Dermaptera from Venezuela. Part 2. Diplatyidae and Labiidae (Geracinae). Revista de la Facultad de Agronomia, Universidad del Zulia 8 (2): 107-125.

Brindle A. 1974c. Dermaptera of Nepal. Senckenbergiana Biologica 55 (1-3): 141-164.

Brindle A. 1975a. A review of the genus Tagalina Dohrn (Dermaptera, Pygidicranidae). Journal of Entomology B 44 (2): 145-151. http://dx.doi.org/10.1111/j.1365-3113.1975.tb00008.x

Brindle A. 1975b. Dermaptera. Revue de Zoologie Africaine 89 (3): 681-695.

Brindle A. 1975c. Ergebnisse der Bhutan-Expedition 1972 des Naturhistorischen Museums in Basel (Dermaptera). Entomologica Basiliensia 1: 1-58.

Brindle A. 1975d. Notes on the neotropical genus Metresura Rehn (Dermaptera, Forficulidae). Entomologist's Monthly Magazine 111: 79-87.

Brindle A. 1976a. Dermaptera from the Seychelles. Revue de Zoologie Africaine 90 (2): 435-445.

Brindle A. 1976b. The Dermaptera of New Caledonia. Pacific Insects 17 (1): 61-86.

Brindle A. 1976c. The Dermaptera of the New Hebrides. Records of the South Australian Museum 17 (13): 221-238. Available from http://www.biodiversitylibrary.org/item/127057\#page/235/mode/1up [accessed 12 Aug. 2015]

Brindle A. 1977a. Dermaptera from Ceylon. Revue Suisse de Zoologie 84 (2): 453-461. Available from http://www.biodiversitylibrary.org/item/129621\#page/487/mode/1up [accessed 12 Aug. 2015]

Brindle A. 1977b. Dermaptera from Venezuela. Part 3. Pygidicranidae and Labiidae (except Geracinae). Revista de la Facultad de Agronomia, Universidad del Zulia 9 (3): 109-131.

Brindle A. 1978. The Dermaptera of Africa. Part II. Annales du Musée royal de l'Afrique Centrale, Zoologie (8) 225: 1-204.

Brindle A. 1979. Five new species of American earwigs (Dermaptera). Entomologist's Monthly Magazine 115: 149-154.

Brindle A. 1980. Dermaptera from the Gunong Mulu National Park, Borneo. The Entomologist's Record and Journal of Variation 92: 172-175. Available from http://www.biodiversitylibrary.org/ item/94533\#page/260/mode/1up [accessed 12 Aug. 2015]

Brindle A. 1981. Two new genera of neotropical earwigs (Dermaptera, Carcinophoridae). Entomologist's Monthly Magazine 117: 77-82. 
Brindle A. 1982a. Dermaptera from Venezuela, Part 4. A new subfamily, a new genus, and new species of Labiidae. Boletín de Entomología Venezolana 2 (4): 33-44.

Brindle A. 1982b. Three new species of African Diplatys Serville (Dermaptera, Diplatyidae). Entomologist's Monthly Magazine 118: 163-166.

Brindle A. 1984. The Esphalmeninae (Dermaptera: Pygidicranidae): a group of Andean and Southern African earwigs. Systematic Entomology 9: 281-292. http://dx.doi.org/10.1111/j.1365-3113.1984. tb00053.x

Brindle A. 1985. Synonymical notes on the American species of Labia Leach (Dermaptera, Labiidae) with a key to species and description of a new species. Entomologist's Monthly Magazine 121: 73-80.

Brindle A. 1987. New Dermaptera records from Nepal, with descriptions of new species and a review of the Himalayan Fauna (Insecta). Courier Forschungsinstitut Senckenberg 93: 333-351.

Brindle A. 1988. Dermaptera at light in Panama. Entomologist's Monthly Magazine 124: 45-56.

Brindle A. 1989. Carcinophora americana (Beauvois) and related species (Derm., Anisolabididae). Entomologist's Monthly Magazine 125: 13-16.

Brindle A. \& Maddison P. [Date not known] The Dermaptera of Fiji, with records from other SW Pacific Islands [unpublished?]. Brindle Archive item 65, Manchester Museum.

Brindle A. \& Oromi P. 1994. Dermaptera. In: Juberthie C. \& Decu V. (eds) Encyclopaedia Biospeologica 1: 289-294.

Burr M. 1901. Auguste de Bormans. The Entomologist's Record and Journal of Variation 13: 85-88. Available from http://www.biodiversitylibrary.org/item/36038\#page/97/mode/1up [accessed 11 Aug. 2015]

Burr M. 1909. Notes on the Forficularia. XV. The Esphalmeninae. Annals and Magazine of Natural History 8 (3): 249-253.

Burr M. 1912. A new species of Arixenia (Dermaptera). Entomologist's Monthly Magazine 48: 105-106. Available from http://www.biodiversitylibrary.org/item/35893\#page/163/mode/1up [accessed 12 Aug. 2015]

Burr M. 1915. On the male genital armature of the Dermaptera. Part II. Psalidae. Transactions of the Royal Microscopical Society 35 (6): 521-546. http://dx.doi.org/10.1111/j.1365-2818.1915.tb01051.x

Burr M. 1917. Contribution à la faune entomologique de l'Indo-Chine Française. Dermaptères. Annales de la Société entomologique de France 86: 57-62. http://www.biodiversitylibrary.org/item/36392\#page/71/ $\underline{\text { mode/lup }}$

Engel M.S. \& Haas F. 2007. Family-group names for earwigs (Dermaptera). American Museum Novitates 3567: 1-20. Available from http://hdl.handle.net/2246/5858 [accessed 12 Aug. 2015]

Gilbert P. 1977. A Compendium of the Biographical Literature on Deceased Entomologists. British Museum (Natural History), London.

Giles E.T. 1973. Dermaptera from the Kermadec Islands with the description of a new species of Labia Leach (Labiidae). New Zealand Entomologist 5: 304-312. http://dx.doi.org/10.1080/00779962.1973.9 $\underline{723027}$

Gorman H.S. 1892. Viaggio di Leonardo Fea in Birmania e regioni vicine. XLVIII. Cleridae. Annali del Museo Genova (2) 12: 718-746. Available from http://www.biodiversitylibrary.org/ item/95179\#page/740/mode/1up [accessed 12 Aug. 2015] 
Harvey J.M.V., Gilbert P. \& Martin K. 1996. A Catalogue of Manuscripts in the Entomology Library of the Natural History Museum, London. Mansell Publishing Limited, London.

Hebard M. 1929. Notes on Malayan and Philippine Dermaptera with descriptions of two new species. Transactions of the The American Entomological Society 55: 335-344.

Hincks W.D. 1938. Die Arthropodenfauna von Madeira nach den Ergebnissen der Reise von Prof. O. Lundblad Juli-August 1935. XI. Dermaptera. Arkiv för Zoologi 30B (12): 1-8.

Hincks W.D. 1940. Dermaptera (earwigs) from Hainan Island. Notes d'Entomologie Chinoise 8 (2): $29-40$.

Hincks W.D. 1947a. A new species of earwig from Sierra Leone (Dermaptera). Entomologist 80: 201203.

Hincks W.D. 1947b. Entomological results from the Swedish Expedition 1934 to Burma and British India, Dermaptera. Arkiv för Zoologi 39A (1): 1-43.

Hincks W.D. 1948. Dermaptera collected in the Ivory Coast by M.M. Paulian and Delamare. Revue Française d'Entomologie 14 (4): 318-321.

Hincks W.D. 1949a. A new genus and species of Dermaptera from Tanganyika. Entomologist's Monthly Magazine 85: 75-77.

Hincks W.D. 1949b. Some earwigs (Dermaptera) from New Zealand. Proceedings of the Royal Entomological Society of London B 18: 201-206. http://dx.doi.org/10.1111/j.1365-3113.1949.tb01410.x

Hincks W.D. 1950a. Some Dermaptera from Kilimanjaro, Tanganyika. Entomologist's Monthly Magazine 86: 179-181.

Hincks W.D. 1950b. Supplementary notes on Mauritian earwigs (Dermaptera). Annals and Magazine of Natural History 12 (3): 353-357. http://dx.doi.org/10.1080/00222935008654058

Hincks W.D. 1951a. Classification, Taxonomy and Distribution of the Dermaptera. Thesis. Hincks Archive No. 484. University of Manchester.

Hincks W.D. 1951b. Notes on some species of Nesogaster (Dermaptera, Labiidae). Annals and Magazine of Natural History 12 (4): 562-578. http://dx.doi.org/10.1080/00222935108654185

Hincks W.D. 1952a. A new Australian species of the genus Antisolabis (Dermaptera, Labiduridae). Entomologist's Monthly Magazine 88: 214-215.

Hincks W.D. 1952b. Some Dermaptera from Sierra Leone. Proceedings of the Royal Entomological Society of London B 21 (1-2): 19-26. http://dx.doi.org/10.1111/j.1365-3113.1952.tb01033.x

Hincks W.D. 1953. Liste preliminaire des dermaptères de Madagascar. Mémoires de l'Institut scientifique de Madagascar, Série E 4: 361-382.

Hincks W.D. 1954a. Obituary. Harry Britten, M.Sc., A.L.S., F.R.E.S. Journal of the Society for British Entomology 4 (9): 225-228.

Hincks W.D. 1954b. La réserve naturelle intégrale du Mont Nimba, II. Dermaptera. Mémoires de l'Institut Français d'Afrique Noire 40: 101-121.

Hincks W.D. 1954c. Notes on Dermaptera. Proceedings of the Royal Entomological Society of London B 23 (9-10): 159-163. http://dx.doi.org/10.1111/j.1365-3113.1954.tb00441.x

Hincks W.D. 1954d. Obituary. Malcolm Burr, M.A., D.Sc., (Oxon), A.R.S.M., F.R.E.S. Entomologist's Monthly Magazine 40: 190-191. 
Hincks W.D. 1954e. The Dermaptera of Sumba and Flores. Verhandlungen der naturforschenden Gesellschaft in Basel 65 (1): 9-23.

Hincks W.D. 1955a. A Systematic Monograph of the Dermaptera of the World. Part I. Pygidicranidae: Diplatyinae. British Museum (Natural History), London.

Hincks W.D. 1955b. Dermaptera. Exploration du Parc national de l'Upemba 35: 3-15.

Hincks W.D. 1955c. New species of pygidicranine earwigs (Dermaptera: Pygidicranidae). Annals and Magazine of Natural History 12 (8): 806-827. http://dx.doi.org/10.1080/00222935508655701

Hincks W.D. 1957a. Dermaptera. Exploration Hydrobiologique du Lac Tanganika 3 (6): 41-42.

Hincks W.D. 1957b. Dermaptera. South African Animal Life 4: 33-94.

Hincks W.D. 1957c. Description of genus Nesolabia. In: Chopard L. (ed.) La Faune Entomologique de l'Île de la Réunion. I. Orthoptéroïdes: 53-56. Mémoires de l'institut scientifique de Madagascar, Série E, vol. 8 .

Hincks W.D. 1958a. Mission du Muséum dans les îles du Golfe de Guinée. Entomologie: X. Dermaptères. Bulletin de la Société entomologique de France 63: 185-186.

Hincks W.D. 1958b. Some notes on Parisolabinae. Eos, Revista Española de Entomología 34: 131-134.

Hincks W.D. 1959. A Systematic Monograph of the Dermaptera of the World. Part II. Pygidicranidae excluding Diplatyinae. British Museum (Natural History), London.

Hincks W.D. 1960a. Indian Dermaptera of the genus Diplatys Serville (Pygidicranidae), with the description of a new species. Bulletin of Entomology 1: 14-15.

Hincks W.D. 1960b. Notes on Dermaptera. Proceedings of the Royal Entomological Society of London B 29 (11-12): 155-159. http://dx.doi.org/10.1111/j.1365-3113.1960.tb01129.x

Hopkins H., Maehr M.D., Haas F. \& Deem L.S. 2014. Dermaptera Species File. Version 5.0/5.0. Available from http://Dermaptera.SpeciesFile.org [accessed 15 Dec. 2014]

Johnson C. 1996. The Manchester Museum Department of Entomology. In: Underwood R. (ed.) The Raven Entomological and Natural History Society, Fifty Years, 1946 to 1996. The Raven Entomological and Natural History Society, Manchester.

Johnson C. 2003. Obituary. Alan Brindle (1915-2001). Entomologist's Monthly Magazine 139: 57.

Johnson K.R. 2003. Karl Jordan: A Life in Systematics. PhD Thesis. Oregon State University, Corvallis, Oregon. Available from http://ir.library.oregonstate.edu/xmlui/handle/1957/30196 [accessed 12 Aug. 2015]

Kitchen T.B. 1962. Obituary. Naturalist 880: 31-32.

Kloet G.S. 1961. Obituary. Walter Douglas Hincks. Entomologist 94 (1179): 181-183.

Kocarek P., Vaclav J. \& Pavel H. 2013. When the body hides the ancestry: Phylogeny of morphologically modified epizoic earwigs based on molecular evidence. PLOS ONE 8 (6): e66900. http://dx.doi. org/10.1371/journal.pone.0066900

Logunov D.V. 2010a. British entomology collections of the Manchester Museum. Journal of the Lancashire \& Cheshire Entomological Society 133-134: 20-44.

Logunov D.V. 2010b. The Manchester Museum's entomology collections. Antenna 34 (4): 163-167.

Logunov D.V. \& Merriman N. (eds) 2012. The Manchester Museum: Window to the World. Third Millenium Ltd., London. 
Marshall A.G. 1977. Interrelationships between Arixenia esau (Dermaptera) and molossid bats and their ectoparasites in Malaysia. Ecological Entomology 2: 285-291. http://dx.doi. org/10.1111/j.1365-2311.1977.tb00893.x

Martin L. 1922. Hans Frühstorfer. Deutsche Entomologische Zeitschrift "Iris" 36 (3/4): 96-103. Available from http://www.biodiversitylibrary.org/item/45761\#page/56/mode/1up [accessed 12 Aug. 2015]

Masaki S. 2006. In Memoriam. Seiroku Sakai (1924-2004). Metaleptea 26 (1): 4-5.

Mogbo T.C. \& Akunne C.E. 2014. Ecological survey of earwigs (Hemimerus talpoides) as ectoparasites of wild African giant rat (Cricetomys gambianus) in Awka, Nigeria. Journal of Natural Sciences Research 4 (18): 36-39.

Nakata S. \& Maa T.C. 1974. A review of the parasitic earwigs (Dermaptera: Arixeniina: Hemimerina). Pacific Insects 16: 307-374.

Natural History Museum. 2015a. Collection Timeline. Available from http://www.nhm.ac.uk/researchcuration/collections/our-collections/invertebrate-collections/arthropod-collections/insect-collections/ orthopteroid-collection/collection-timeline/index.html [accessed 30 Mar. 2015]

Natural History Museum. 2015b. Orthopteroid Collection Statistics. Available from http://www.nhm. ac.uk/research-curation/collections/our-collections/invertebrate-collections/arthropod-collections/ insect-collections/orthopteroid-collection/orthopteriod-collection-statistics/index.html [accessed 23 Mar. 2015]

Natural History Museum. 2015c. Tring. Available from http://www.nhm.ac.uk/visit/tring.html [accessed 8 Jul. 2015]

Noyes J.S. 2003. Obituary, Geoffrey John Kerrich (1909-2003). Entomologist’s Monthly Magazine 139: $125-129$.

Pryce D. \& White L. 2014. Labidura herculeana. The IUCN Red List of Threatened Species. Version 2014.3. Available from www.iucnredlist.org [accessed 23 Feb. 2015]

Ramamurthi B.N. 1960. Dermaptera from Nilgiri and Kodaikanal Hills with description of a new species. Bulletin of Entomology 1: 34-37.

Rentz D. 2014. A Guide to the Cockroaches of Australia. CSIRO Publishing, Clayton, Victoria.

Reports. 1890-2003. The Manchester Museum. The University, Manchester.

Sakai S. 1982. A new proposed classification of the Dermaptera with special reference to the check list of the Dermaptera of the world. Bulletin of Daito Bunka University 20: 1-108.

Srivastava G.K. 1982. Notes on a collection of Dermaptera (Insecta) present in the Natural History Museum, Basel. Entomologica Basiliensia 7: 61-76.

Steinmann H. 1974. A new generic classification of the species group of Diplatys Serville (Dermaptera: Pygidicranidae). Acta Zoologica Academiae Scientiarum Hungaricae 20 (1-2): 187-205.

Steinmann H. 1975. Suprageneric classification of Dermapatera. Acta Zoologica Academiae Scientiarum Hungaricae 21: 195-220.

Steinmann H. 1978. A revision of the African Gonolabis Burr, 1910 species (Dermaptera, Carcinophoridae). Folia Entomologica Hungarica 31: 189-197.

Steinmann H. 1979. A revision of the genus Aborolabis Srivastavai, 1969. Eos, Revista Española de Entomología 53: 213-222. 
Steinmann H. 1980. A revision of the species belonging in Forcipula Bolivar, 1897 (Dermaptera: Labiduridae). Acta Zoologica Academiae Scientiarum Hungaricae 26: 229-252.

Steinmann H. 1982a. Synopsis of Dermaptera of the World. Part I: Family Pygidicranidae (I). Entomologische Abhandlungen des Staatliches Museums für Tierkunde Dresden 45: 195-211.

Steinmann H. 1982b. Revision of the genus Timomenus Burr, 1907 (Dermaptera: Forficulidae). Acta Zoologica Academiae Scientiarum Hungaricae 28 (3-4): 361-368.

Steinmann H. 1986a. A new generic classification for the Diplatys species-groups (Dermaptera: Pygidicranidae). Acta Zoologica Academiae Scientiarum Hungaricae 32(1-2):169-179.

Steinmann H. 1986b. Dermaptera, Part I: Catadermaptera (I): Pygidicranidae. Das Tierreich 102: 1-336.

Steinmann H. 1987. Two new genera and species for the subfamily Labiinae (Dermaptera: Labiidae). Acta Zoologica Academiae Scientiarum Hungaricae 33 (1-2): 177-186.

Steinmann H. 1989a. Dermaptera, Part II: Catadermaptera (II): Carcinophoridae, Labiduridae, and Apachyidae. Das Tierreich 105: 1-495.

Steinmann H. 1989b. Dermaptera, Part III: Eudermaptera (I): Labiidae. Das Tierreich 106: 1-550.

Steinmann H. 1989c. World Catalogue of Dermaptera. Series Entomologica vol. 43. Kluwer Academic Publishers, Dordrecht, the Netherlands.

Steinmann H. 1993. Dermaptera, Part IV: Eudermaptera (II): Chelisochidae and Forficulidae. Das Tierreich 108: 1-711.

Thimann R.G. 1961. Reminiscences of W.D. Hincks. The Bulletin of the Amateur Entomologists 'Society 20 (250): 113-114.

Veth P.J. 1892. Midden-Sumatra. Reizen en Onderzoekingen der Sumatra-Expeditie, uitgerust door het Aardrijkskundig Genootschap, 1877-1879 vol. 4 (1, 2). E.J. Brill, Leiden. Available from http://www. biodiversitylibrary.org/item/102928\#page/7/mode/1up [accessed 18 Aug. 2015]

Wentholt A. \& Wilschut J. 2003. In Kaart gebracht met Kapmes en Kompas: Expedities en Onderzoekstochten 1873-1960. Available from http://www.knag-expedities.nl/ [accessed 31 Mar. 2015]

Manuscript received: 29 April 2015

Manuscript accepted: 13 July 2015

Published on: 1 October 2015

Topic editor: Koen Martens

Desk editor: Danny Eibye-Jacobsen

Printed versions of all papers are also deposited in the libraries of the institutes that are members of the EJT consortium: Muséum National d'Histoire Naturelle, Paris, France; Botanic Garden Meise, Belgium; Royal Museum for Central Africa, Tervuren, Belgium; Natural History Museum, London, United Kingdom; Royal Belgian Institute of Natural Sciences, Brussels, Belgium; Natural History Museum of Denmark, Copenhagen, Denmark. 


\section{Appendix I: Correspondence in the Hincks Archive relating to Dermaptera, the Manchester Museum}

Ordered alphabetically by correspondent, then date.

\begin{tabular}{|c|c|c|c|}
\hline Item No. & From & Date & No. of pages \\
\hline 387 & G. Bey-Bienko & 24 Jan. 1937 & postcard \\
\hline 90 & G. Bey-Bienko & 17 Mar. 1937 & 1 \\
\hline 72 & Alfredo Borelli, Torino & 16 Feb. 1933 & 1 \\
\hline 52 & M. Burr, Maidenhead & 24 Jan. 1933 & 2 \\
\hline 54 & M. Burr, Maidenhead & 02 Feb. 1933 & 3 \\
\hline 55 & M. Burr, Maidenhead & 09 Feb. 1933 & 1 \\
\hline 53 & M. Burr, Maidenhead & 22 Feb. 1933 & 1 \\
\hline 51 & M. Burr, Maidenhead & 21 Jan. 1934 & 1 \\
\hline 57 & M. Burr, Maidenhead & 24 Apr. 1934 & 1 \\
\hline 49 & M. Burr, Maidenhead & 23 Oct. 1934 & 1 \\
\hline 50 & M. Burr, Maidenhead & 25 Nov. 1934 & 1 \\
\hline 79 & M. Burr, Windsor & 23 Mar. 1935 & 1 \\
\hline 62 & M. Burr & 30 Mar. 1935 & 1 \\
\hline 63 & M. Burr, Windsor & 13 May 1935 & 3 \\
\hline 64 & M. Burr, Windsor & 02 Oct. 1935 & 1 \\
\hline 66 & M. Burr, Windsor & 07 Oct. 1935 & 1 \\
\hline 67 & M. Burr, Windsor & 31 Oct. 1935 & 1 \\
\hline 74 & M. Burr, enclosing letter from O. Piel, Shanghai & 10 Nov. 1935 & 2,1 \\
\hline 68 & M. Burr, Windsor & 12 Nov. 1935 & 1 \\
\hline 77 & M. Burr, Maidenhead & 30 Jan. 1936 & 2 \\
\hline 80 & M. Burr, Windsor & 14 Feb. 1936 & 2 \\
\hline 82 & M. Burr, Windsor & 07 Mar. 1936 & 3 \\
\hline 86 & M. Burr, Windsor & 26 Sep. 1936 & 2 \\
\hline 87 & M. Burr, Windsor & 17 Dec. 1936 & 1 \\
\hline 88 & M. Burr, Windsor & 06 Jan. 1937 & 1 \\
\hline 96 & M. Burr, Windsor & 11 Mar. 1937 & $1+3$ photos \\
\hline 99 & M. Burr, Worthing & 13 Jun. 1938 & 2 \\
\hline 103 & M. Burr, Worthing & 14 Jul. 1938 & 1 \\
\hline 109 & M. Burr, Worthing & 28 Aug. 1938 & 1 \\
\hline 110 & M. Burr, Worthing & 15 Nov. 1938 & 1 \\
\hline 120 & M. Burr to N.D. Riley, Natural History Museum & 15 Nov. 1938 & 1 \\
\hline 111 & M. Burr, Worthing & 17 Nov. 1938 & 1 \\
\hline 69 & M. Burr, Windsor & 04 May 1939 & 3 \\
\hline 43 & M. Burr, Istanbul & 19 Dec. 1948 & 1 \\
\hline 42 & M. Burr, Istanbul & 10 Sep. 1950 & 1 \\
\hline 41 & $\begin{array}{l}\text { M. Burr, Istanbul, } \\
\text { W.D. Hincks }\end{array}$ & $\begin{array}{l}08 \text { Oct. } 1951, \\
12 \text { Dec. } 1951\end{array}$ & 1,1 \\
\hline
\end{tabular}


MILES C., Dermaptera of the Manchester Museum

\section{Appendix I, continued}

\begin{tabular}{|c|c|c|c|}
\hline Item No. & From & Date & No. of pages \\
\hline 389 & Unknown person [M. Burr?] & No date & postcard \\
\hline 390 & Fritz Carpentier & 15 Sep. 1936 & postcard \\
\hline 276 & $\begin{array}{l}\text { Lucien Chopard, Muséum National d'Histoire Naturelle } \\
\text { Entomologie, Paris }\end{array}$ & 10 Feb. 1960 & 1 \\
\hline 275 & W.D. Hincks to L. Chopard & 07 Mar. 1960 & 1 \\
\hline 274 & $\begin{array}{l}\text { Lucien Chopard, Muséum National d'Histoire Naturelle } \\
\text { Entomologie, Paris }\end{array}$ & 15 Mar. 1960 & 1 \\
\hline 271 & $\begin{array}{l}\text { Lucien Chopard, Muséum National d'Histoire Naturelle } \\
\text { Entomologie, Paris }\end{array}$ & 30 Nov. 1960 & 1 \\
\hline 272 & W.D. Hincks to L. Chopard & 05 Dec. 1960 & 1 \\
\hline 268 & $\begin{array}{l}\text { Lucien Chopard, Muséum National d'Histoire Naturelle } \\
\text { Entomologie, Paris }\end{array}$ & 16 Jan. 1961 & 1 \\
\hline 269 & W.D. Hincks to L. Chopard & 25 Jan. 1961 & 1 \\
\hline 39 & $\begin{array}{l}\text { Ashley B. Gurney, Divn. of Insect Identification, US Dept of } \\
\text { Agriculture, Washington }\end{array}$ & 03 May 1950 & 1 \\
\hline 38 & $\begin{array}{l}\text { Ashley B. Gurney, Divn. of Insect Identification, US Dept of } \\
\text { Agriculture, Washington }\end{array}$ & 27 Mar. 1951 & 3 \\
\hline 125 & $\begin{array}{l}\text { Ashley B. Gurney, Divn. of Insect Identification, US Dept of } \\
\text { Agriculture, Washington }\end{array}$ & 06 Sep. 1960 & 3 \\
\hline 127 & $\begin{array}{l}\text { [Probably Ashley B. Gurney, Divn. of Insect Identification, } \\
\text { US Dept of Agriculture, Washington] }\end{array}$ & 06 Sep. 1960 & 1 \\
\hline 128 & [Probably W.D. Hincks to Ashley B. Gurney] & 29 Sep. 1960 & 1 \\
\hline 40 & $\begin{array}{l}\text { Kenneth J. Hayward, Director, Instituto de Entomología, } \\
\text { Tucumán, Argentina }\end{array}$ & 29 Nov. 1949 & 3 \\
\hline 247 & W.D. Hincks to Colin Johnson & 31 Aug. 1960 & 1 \\
\hline 239 & W.D. Hincks to P.V. Joshi & 20 Oct. 1960 & 1 \\
\hline 237 & $\begin{array}{l}\text { P.V. Joshi, Dept. of Zoology, N. Wadia College, Poona, } \\
\text { India }\end{array}$ & 31 Oct. 1960 & 1 \\
\hline 283 & $\begin{array}{l}\text { Dr. A.P. Kapur, Officer in Charge, Entomology Secn., } \\
\text { Zoological Survey of India, Calcutta }\end{array}$ & 26 Nov. 1959 & 1 \\
\hline 280 & $\begin{array}{l}\text { Dr. A.P. Kapur, Officer in Charge, Entomology Secn., } \\
\text { Zoological Survey of India, Calcutta }\end{array}$ & 21 Mar. 1960 & 1 \\
\hline 281 & $\begin{array}{l}\text { W.D. Hincks to Dr. A.P. Kapur, Officer in Charge, } \\
\text { Entomology Secn., Zoological Survey of India, Calcutta }\end{array}$ & 24 Mar. 1960 & 1 \\
\hline 279 & $\begin{array}{l}\text { W.D. Hincks to Dr. A.P. Kapur, Officer in Charge, } \\
\text { Entomology Secn., Zoological Survey of India, Calcutta }\end{array}$ & 28 Mar. 1960 & 1 \\
\hline 278 & $\begin{array}{l}\text { Dr. A.P. Kapur, Officer in Charge, Entomology Secn., } \\
\text { Zoological Survey of India, Calcutta }\end{array}$ & 15 Apr. 1960 & 1 \\
\hline 277 & $\begin{array}{l}\text { W.D. Hincks to Dr. A.P. Kapur, Officer in Charge, } \\
\text { Entomology Secn., Zoological Survey of India, Calcutta }\end{array}$ & 19 Jul. 1960 & 1 \\
\hline 310 & Dr. H.W. Koepcke, Peru & 17 Nov. 1959 & 1 \\
\hline 309 & W.D. Hincks to Dr. H.W. Koepcke, Peru & 23 Nov. 1959 & 1 \\
\hline 359 & A. Loveridge, M/V Durban Castle, in the Thames & 15 Jun. 1960 & 1 \\
\hline 360 & W.D. Hincks to A. Loveridge & 23 Jun. 1960 & 1 \\
\hline
\end{tabular}




\section{Appendix I, continued}

\begin{tabular}{|c|c|c|c|}
\hline Item No. & From & Date & No. of pages \\
\hline 317 & A. Loveridge & 01 Dec. 1960 & 1 \\
\hline 318 & W.D. Hincks to A. Loveridge & 05 Jan. 1961 & 1 \\
\hline 70 & H.K. Long, Shell Petroleum Company of Colombia, Bogota & 26 Mar. 1939 & 1 \\
\hline 71 & H.K. Long, Shell Petroleum Company of Colombia, Bogota & 04 Jul. 1939 & 1 \\
\hline 106 & H. Lucht, Bondowoso, Java & 17 Jun. 1938 & 1 \\
\hline 46 & H. Lucht, Bondowoso, Java & 17 Aug. 1938 & 2 \\
\hline 47 & H. Lucht, Bondowoso, Java & 24 May 1939 & 1 \\
\hline 37 & Raymond Mamet, Mauritius & 09 Nov. 1949 & 1 \\
\hline 35 & $\begin{array}{l}\text { T. McCarthy, Chief Entomologist N.S.W. Dept. of } \\
\text { Agriculture }\end{array}$ & 03 Apr. & 1 \\
\hline 85 & C. Menozzi & 25 Jun. 1936 & postcard \\
\hline 75 & E. Morales Agacino, Madrid & 11 Jan. 1936 & 1 \\
\hline 78 & E. Morales Agacino, Madrid & 27 Jan. 1936 & postcard \\
\hline 93 & Edward P. Mumford, Dept of Entomology, Oxford & 24 Sep. 1937 & 2 \\
\hline 94 & Edward P. Mumford, Dept of Entomology, Oxford & 24 Sep. 1937 & 1 \\
\hline 102 & Ferd. Nevermann, San Jose, Costa Rica & 11 Apr. 1938 & 1 \\
\hline 92 & R.P.O. Piel, Director, Musée Heude, Shanghai & 28 Feb. 1937 & 2 \\
\hline 73 & R.P.O. Piel, Director, Musée Heude, Shanghai & 03 Jan. 1936 & 1 \\
\hline 89 & R.P.O. Piel, Director, Musée Heude, Shanghai & 30 Nov. 1936 & 1 \\
\hline 240 & David R. Ragge & 12 Oct. 1960 & 1 \\
\hline 56 & $\begin{array}{l}\text { James A. Rehn, Acting Curator, Academy of Natural } \\
\text { Sciences of Philadelphia }\end{array}$ & 03 Mar. 1933 & 1 \\
\hline 58 & $\begin{array}{l}\text { James A. Rehn, Acting Curator, Academy of Natural } \\
\text { Sciences of Philadelphia }\end{array}$ & 04 Apr. 1933 & 1 \\
\hline 34 & Isabel Sanabria & 18 May 1959 & 2 \\
\hline 124 & F.H. Schade, Villarrica, Paraguay & 25 Feb. 1939 & 1 \\
\hline 104 & F.H. Schade, Villarrica, Paraguay & 19 Jun. 1938 & 1 \\
\hline 59 & $\begin{array}{l}\text { Dr H. Schouteden, Director Musée du Congo Belge, } \\
\text { Tervueren, Belgium }\end{array}$ & 02 Feb. 1935 & 1 \\
\hline 60 & $\begin{array}{l}\text { Dr H. Schouteden, Director Musée du Congo Belge, } \\
\text { Tervueren, Belgium. }\end{array}$ & 22 Feb. 1935 & 1 \\
\hline 95 & $\begin{array}{l}\text { V. Van Straelen, President, Institut des Parcs nationaux du } \\
\text { Congo Belge, Brussels }\end{array}$ & 22 Jul. 1937 & 1 \\
\hline 105 & M.E. Walsh, Soekaboemi, Java & 17 Jun. 1938 & 1 \\
\hline 45 & M.E. Walsh, Soekaboemi, Java & 16 Aug. 1938 & 2 \\
\hline 48 & Dr. Friedrich Zacher, Berlin & 20 Nov. 1934 & 1 \\
\hline 76 & $\begin{array}{l}\text { Unknown person, Musée du Congo Belge, Tervuren, } \\
\text { Belgium }\end{array}$ & 20 Jan. 1935 & 1 \\
\hline 386 & Unknown person & 22 Mar. 1938 & postcard \\
\hline 375 & Unknown person [Letterhead 'F. H. Walz, Buenos Aires'] & 22 Jun. 1961 & 1 \\
\hline
\end{tabular}


MILES C., Dermaptera of the Manchester Museum

\section{Appendix II: Correspondence in the Brindle Archive relating to Dermaptera, the Manchester Museum}

Ordered alphabetically by correspondent, then date.

\begin{tabular}{|c|c|c|c|}
\hline Item No. & From & Date & No. of pages \\
\hline 147 & $\begin{array}{l}\text { Annette Aiello regarding publication of 'Insects of Panama } \\
\text { and Mesoamerica: Selected Studies' }\end{array}$ & 12 May 1992 & \\
\hline 148 & A. Brindle to A. Aiello & 27 May 1992 & \\
\hline 18 & M. Beier & 15 Nov. 1963 & postcard \\
\hline 242 & $\begin{array}{l}\text { Dr. T. Cekalovic (in Spanish) regarding supply of Dermaptera } \\
\text { from Chile, Easter Islands, Juan Fernández Island, Magellan } \\
\text { region and Tierra del Fuego }\end{array}$ & 08 Nov. 1974 & 2 \\
\hline 243 & Handwritten translation of 242 & & 1 \\
\hline 247 & $\begin{array}{l}\text { Dr. T. Cekalovic, sending } 1 \text { box Dermaptera and Isoptera, } 2 \\
\text { boxes Lepidoptera }\end{array}$ & 20 Feb. 1975 & 1 \\
\hline 248 & Handwritten translation of 243 & & 1 \\
\hline 249 & Dr. T. Cekalovic & 03 Apr. 1975 & 1 \\
\hline 259 & $\begin{array}{l}\text { A. Brindle to Dr. T. Cekalovic, with certificate of posting for } \\
\text { return of insects }\end{array}$ & 15 Oct. 1975 & 3 \\
\hline 55 & Karl Jordan to W.D. Hincks & 30 Jan. 1947 & 1 \\
\hline 154 & $\begin{array}{l}\text { Dr. H.W. Koepcke, Peru to W.D. Hincks regarding progress } \\
\text { on identification }\end{array}$ & 12 Mar. 1964 & 1 \\
\hline 155 & Dr. H.W. Koepcke's reply to 154 & 22 Jul. 1964 & 1 \\
\hline 157 & $\begin{array}{l}\text { Dr. H.W. Koepcke regarding Brindle's naming of Idolopsalis } \\
\text { koepckei }\end{array}$ & 10 Nov. 1965 & 1 \\
\hline 158 & $\begin{array}{l}\text { A. Brindle to Dr. H.W. Koepcke enclosing paper reprints } \\
\text { relating to Dermaptera sent for identification plus list of } \\
\text { Peruvian Dermaptera }\end{array}$ & 05 Jul. 1967 & 5 \\
\hline 218 & P.S. Nathan, sending 10 boxes of 'desiderata' & 21 Jul. 1965 & 1 \\
\hline 219 & A. Brindle's to P.S. Nathan, reply to 218 & 08 Sep. 1965 & 1 \\
\hline 220 & P.S. Nathan, enquiring what families Brindle would like & 24 Nov. 1965 & 1 \\
\hline 221 & P.S. Nathan & 22 Aug. 1966 & 1 \\
\hline 223 & A. Brindle to P.S. Nathan & 08 Jul. 1967 & 1 \\
\hline 222 & P.S. Nathan & 23 Nov. 1967 & 1 \\
\hline 224 & P.S. Nathan, sending 3 boxes of Dermapera and beetles & 13 Feb. 1968 & 1 \\
\hline 225 & $\begin{array}{l}\text { Attached to 226. Notification of retirement of P.S. Nathan, } \\
\text { handing over of business to his daughter-in-law }\end{array}$ & & 1 \\
\hline 226 & $\begin{array}{l}\text { See 225. Mrs T.R.S. Nathan, sending } 1 \text { box of Dermaptera } \\
\text { and receipt of reprint of earwig named for P.S. Nathan }\end{array}$ & 02 Jul. 1969 & 1 \\
\hline 227 & Mrs T.R.S. Nathan & 15 Apr. 1970 & 1 \\
\hline 228 & $\begin{array}{l}\text { A. Brindle to Mrs T.R.S. Nathan, requesting more } \\
\text { Dermaptera, Ptiliidae, Neuroptera or Orthoptera }\end{array}$ & 22 Apr. 1970 & 1 \\
\hline 229 & Mrs T.R.S. Nathan & 31 Mar. 1971 & 1 \\
\hline 230 & $\begin{array}{l}\text { A. Brindle to Mrs T.R.S. Nathan, requesting further } \\
\text { Dermaptera, Neuroptera, Orthoptera, Hemiptera and } \\
\text { Hymenoptera }\end{array}$ & 14 Apr. 1971 & 1 \\
\hline
\end{tabular}




\section{Appendix II, continued}

\begin{tabular}{|c|c|c|c|}
\hline Item No. & From & Date & No. of pages \\
\hline 231 & $\begin{array}{l}\text { Mrs T.R.S. Nathan, sending } 3 \text { boxes Dermapera and } \\
\text { Orthoptera }\end{array}$ & 12 Jul. 1971 & 1 \\
\hline 232 & Mrs T.R.S. Nathan & 27 Apr. 1972 & 1 \\
\hline 233 & $\begin{array}{l}\text { Mrs T.R.S. Nathan, sending } 4 \text { double size boxes of } \\
\text { Dermaptera, Orthoptera, Hemiptera and Hymenoptera }\end{array}$ & 13 Jul. 1972 & 1 \\
\hline 234 & A. Brindle to Mrs T.R.S. Nathan & 18 Sep. 1972 & 1 \\
\hline 61 & $\begin{array}{l}\text { P. Oromi with some additions and amendments to Brindle's } \\
\text { article on cavernicole Dermaptera (No. 63) and Brindle's } \\
\text { reply }\end{array}$ & $\begin{array}{l}30 \text { Oct. } 1990 \\
08 \text { Nov. } 1990\end{array}$ & 2 \\
\hline 38 & Edward [Popham?] & 17 Aug. 1981 & 1 \\
\hline 235 & $\begin{array}{l}\text { A. Brindle to S.K. Sircar, Assam, regarding supply of } \\
\text { Dermaptera and Ptiliidae }\end{array}$ & 29 Nov. 1967 & 1 \\
\hline 236 & S.K. Sircar, Assam, reply to 235 & 09 Dec. 1967 & 1 \\
\hline 237 & A. Brindle to S.K. Sircar & 05 Jan. 1968 & 1 \\
\hline 238 & S.K. Sircar, sending 700 earwigs & 27 Feb. 1968 & 1 \\
\hline 239 & A. Brindle to S.K. Sircar & 04 Mar. 1968 & 1 \\
\hline 174 & $\begin{array}{l}\text { A. Brindle to Dr R. zur Strassen regarding return of } \\
\text { Cassidinae unidentified }\end{array}$ & 24 Jan. 1973 & 1 \\
\hline 175 & Dr. R. zur Strassen's reply to 174 & 05 Feb. 1973 & 1 \\
\hline 272 & $\begin{array}{l}\text { A. Brindle to Taiwan Novelty Company, enquiring about } \\
\text { supply of Dermaptera from Formosa }\end{array}$ & 29 Nov. 1967 & 1 \\
\hline 273 & $\begin{array}{l}\text { Au Chun-Cheng, Director, Taiwan Novelty Company, reply to } \\
272\end{array}$ & 07 Dec. 1967 & 1 \\
\hline 262 & J.S. Taylor, Natal, South Africa & 16 Feb. 1966 & 1 \\
\hline 263 & J.S. Taylor, Natal, South Africa & 15 Mar. 1966 & 1 \\
\hline 264 & $\begin{array}{l}\text { J.S. Taylor, Natal, South Africa, sending vials of insects } \\
\text { including forficulids and staphylinids }\end{array}$ & 04 Apr. 1966 & 1 \\
\hline 274 & $\begin{array}{l}\text { A. Brindle to H. Winkler, Vienna, requesting prices for } \\
\text { Coleoptera, Dermaptera and other small orders }\end{array}$ & 21 Nov. 1968 & 1 \\
\hline
\end{tabular}

